\title{
Nanocharacterization of magnetoresistant oxide tunnel barrier structures
}

\author{
Dissertation \\ zur Erlangung des Doktorgrades \\ der Mathematisch-Naturwissenschaftlichen Fakultäten \\ der Georg-August-Universität zu Göttingen
}

vorgelegt von

Mario Kuduz

aus Göttingen

Göttingen 2004 
D7

Referent: Prof. Dr. Reiner Kirchheim

Korreferent: Prof. Dr. Guido Schmitz

Tag der mündlichen Prüfung: 05. Oktober 2004 


\section{Logic is the beginning of wisdom; not the end.}

Spock in "Star Trek VI - The undiscovered country" 


\section{Contents}

\begin{tabular}{lll}
\hline 1 & Introduction & 7 \\
\hline
\end{tabular}

\begin{tabular}{|lll}
\hline 2 & Theoretical background & 9
\end{tabular}

2.1 Magneto-resistance - XMR devices . . . . . . . . . . . . . . . . . 9

2.1.1 Interlayer Exchange Coupling (IEC) and the RKKY Model . . 9

2.1 .2 Giant Magneto-Resistance (GMR) . . . . . . . . . . . . . . . 12

2.1 .3 Tunnel Magneto-Resistance (TMR) . . . . . . . . . . . . . . . 13

The Jullière model . . . . . . . . . . . . . . . . . . . . . . . 13

The Slonczewski model . . . . . . . . . . . . . . . . . . . . . . 14

Jullière and Slonczewski; a final conclusion . . . . . . . . . . . 17

2.2 Derivation of barrier properties . . . . . . . . . . . . . . . . 17

The Brinkman model . . . . . . . . . . . . . . . . . . . 17

2.3 Investigations of tunnel barrier structures . . . . . . . . . . . . . . 20

\begin{tabular}{lll}
\hline Experimental methods & 25
\end{tabular}

3.1 Sample preparation . . . . . . . . . . . . . . . . . 25

$3.1 .1 \quad$ Samples for FIM- and TAP-applications . . . . . . . . . . . 25

3.1 .2 Planar samples . . . . . . . . . . . . . . . . . 25

Specimen for TEM-analysis . . . . . . . . . . . . . . . . 25

Specimen for SIMS- and electrical characterization . . . . . . 27

3.2 Ion beam sputter deposition . . . . . . . . . . . . . . . . . . . 29

$3.3 \quad$ Field Ion Microscopy and 3D atom-probe tomography . . . . . . . . . 30

3.3.1 $\quad$ Field ion microscopy $(\mathrm{FIM})$. . . . . . . . . . . . . . . . 31

$3.3 .2 \quad 3 \mathrm{D}$ atom-probe tomography (TAP) . . . . . . . . . . 33 
3.4 Data analysis . . . . . . . . . . . . . . . . . 36

3.4.1 Reconstruction procedure for layered structures . . . . . . . . 36

3.4 .2 Concentration profiles . . . . . . . . . . . . . . . 37

3.4 .3 Derivation of the Gibbsian excess . . . . . . . . . . . . . . . . . 39

3.5 Additional analysis methods . . . . . . . . . . . . . . . . . . . . 39

3.5.1 Transmission-electron-microscopy (TEM) . . . . . . . . . . . . 39

3.5 .2 Secondary-ion-mass-spectrometry (SIMS) . . . . . . . . . . . . 40

3.5.3 Measurement of electrical properties and TMR . . . . . . . . . 42

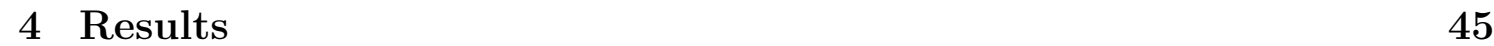

4.1 TAP results . . . . . . . . . . . . . . . . . . . . . 45

$4.1 .1 \quad$ As-prepared state . . . . . . . . . . . . . . . . . . 45

4.1 .2 Annealed state .................... 52

4.2 TEM results . . . . . . . . . . . . . . . . . . . . . . . . . . . . . . 62

$4.3 \quad$ SIMS results $\ldots \ldots \ldots \ldots$. . . . . . . . . . . . . . . . . . 62

4.4 Electrical properties and TMR . . . . . . . . . . . . . . . . . 62

\begin{tabular}{lll}
\hline 5 & Discussion & 71
\end{tabular}

5.1 As-prepared state . . . . . . . . . . . . . . . . . 71

5.2 Annealed state . . . . . . . . . . . . . . . . . . 74

5.2.1 $\quad$ Phenomenological description . . . . . . . . . . . . . . 74

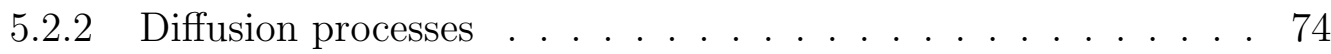

$5.3 \quad$ Electrical properties and TMR . . . . . . . . . . . . . . . . . . . . . 79

$\begin{array}{llr}6 & \text { Summary and Outlook } & 81\end{array}$

\begin{tabular}{ll}
\hline List of tables & 83
\end{tabular}

\begin{tabular}{ll}
\hline List of figures & 85
\end{tabular}

\begin{tabular}{lr}
\hline Bibliography & 89
\end{tabular}

\begin{tabular}{|ll}
\hline Aknowledgements & 91
\end{tabular}

\begin{tabular}{lr}
\hline Index & 94
\end{tabular} 


\section{Chapter 1}

\section{Introduction}

Tunnel-barriers used in Tunnel magneto-Resistance devices (TMR) are currently of interest for application in sensors, storage devices and disc drive reading heads. Compared to Giant-Magneto Resistance (GMR) devices, tunnel-barriers are distinguished by an improved effect amplitude and a higher base resistivity so that they may be used in 'current perpendicular to plane' arrangements.

In this work the nano-structure of such TMR devices is investigated by means of field ion microscopy in combination with a high atomic resolution 2D detection setup. TMR structures consisting of $\mathrm{Co}$ and $\mathrm{Ni}_{79} \mathrm{Fe}_{21}$ (=Permalloy, Py) electrodes and $\mathrm{Al}_{2} \mathrm{O}_{3}$ barriers were prepared by ion beam sputter deposition on tips of only 30 to $50 \mathrm{~nm}$ radius of curvature suitable for field ion microscopy. The 3D spatial distribution of the atomic species is determined with sub-nanometer resolution. In particular, the dependence of the oxygen distribution on various deposition methods, i.e. deviations from the ideal stoichiometry within the barrier and partial oxidation of the metallic electrodes is discussed. Additionally the dependence on different annealing temperatures of the structure of the so-called TMR spin-valve structures is investigated. The derived results are verified by application of further analysis methods like SIMS, TEM, and electrical contact experiments in order to obtain data concerning magnetic properties.

The knowledge of physical processes on nanometer scales in TMR devices is important in order to understand the behavior of the materials used under different circumstances and hence to optimize and improve devices in preparation for a serial fabrication in the near future. Although there are alternatives to the ion beam sputter deposition for producing adequate oxide barrier structures in TMR setups - e.g. magnetron sputtering or plasma oxidation - the derived results are supposed to be transferable to all TMR systems. Up to now, ion beam sputter deposition has been the only way to produce samples adequate for atom probe tomography which is a measuring technique, based on field ion microscopy. Electrical contact experiments have shown that the deposited samples show TMR properties.

For the first time, in this work non-conductive oxide barriers have been measured and characterized by atom probe tomography although one might expect that a this 
technique is only applicable on conductive materials. The performed measurements have been successfully measured if the non-conductive layer does not exceed a thickness of $2.5 \mathrm{~nm}$. Thus, the results presented in this work show new possibilities for applications in atom probe tomography in the near future.

Compared to high resolution transmission electron microscopy (HRTEM), atom probe tomography provides a higher resolution and a detailed chemical analysis. Additionally, atom probe tomography is not limited by projection effects. Hence, by applying atom probe tomography, a detailed analysis in deeper regions of the samples are possible.

Chapter 2 describes the theoretical background required for a physical description of the phenomena. In chapter 3 , the experimental methods are introduced. An overview and description of the experimental results is given in chapter 4 while in chapter 5 the experimental results are interpreted and analyzed in more detail. Chapter 6 summarizes this work and additionally gives an outlook to further useful investigations. 


\section{Chapter 2}

\section{Theoretical background}

\subsection{Magneto-resistance - XMR devices}

The main motivation for these investigations is to get a better physical understanding of the processes taking place on atomic scales in TMR devices. TMR devices are a further development of the GMR setups currently used in storage devices, diskdrive heads and sensors. TMR devices can provide a higher resolution in resistance and therefore a higher storage capacity for a given volume.

In TMR and GMR devices the resistance of each device is influenced by modifying the magnetization of the electrodes that are separated by each other by a non-ferromagnetic but conductive layer in case of the GMR and a non-conductive insulating layer in case of the TMR, respectively.

\subsubsection{Interlayer Exchange Coupling (IEC) and the RKKY Model}

Magnetization in ferromagnetic materials is caused by an imbalance of the spin density of states (DOS). In a non-ferromagnetic material, the DOS of spin-up and spin-down electrons is equal to each other while in a ferromagnetic material the status of one of the spin orientations is shifted to a higher energy level. The magnetization is defined by the difference of the amount of spin-up and spin-down electrons. In Fig. 2.1 the spin-down band is fully occupied while the spin-up band is occupied only partly because it is shifted to a higher energy level and hence parts of the spin-up band are located beyond the Fermi-energy. In case of Fig. 2.1 the spin-down electrons represent the majority-electrons while the minority-electrons are represented by the spin-up electrons.

In a ferromagnetic material of a certain size, magnetic domains characterized by their magnetization ${ }^{1}$ are created in response to the fringing field surrounding each

\footnotetext{
${ }^{1}$ parallel or antiparallel
} 


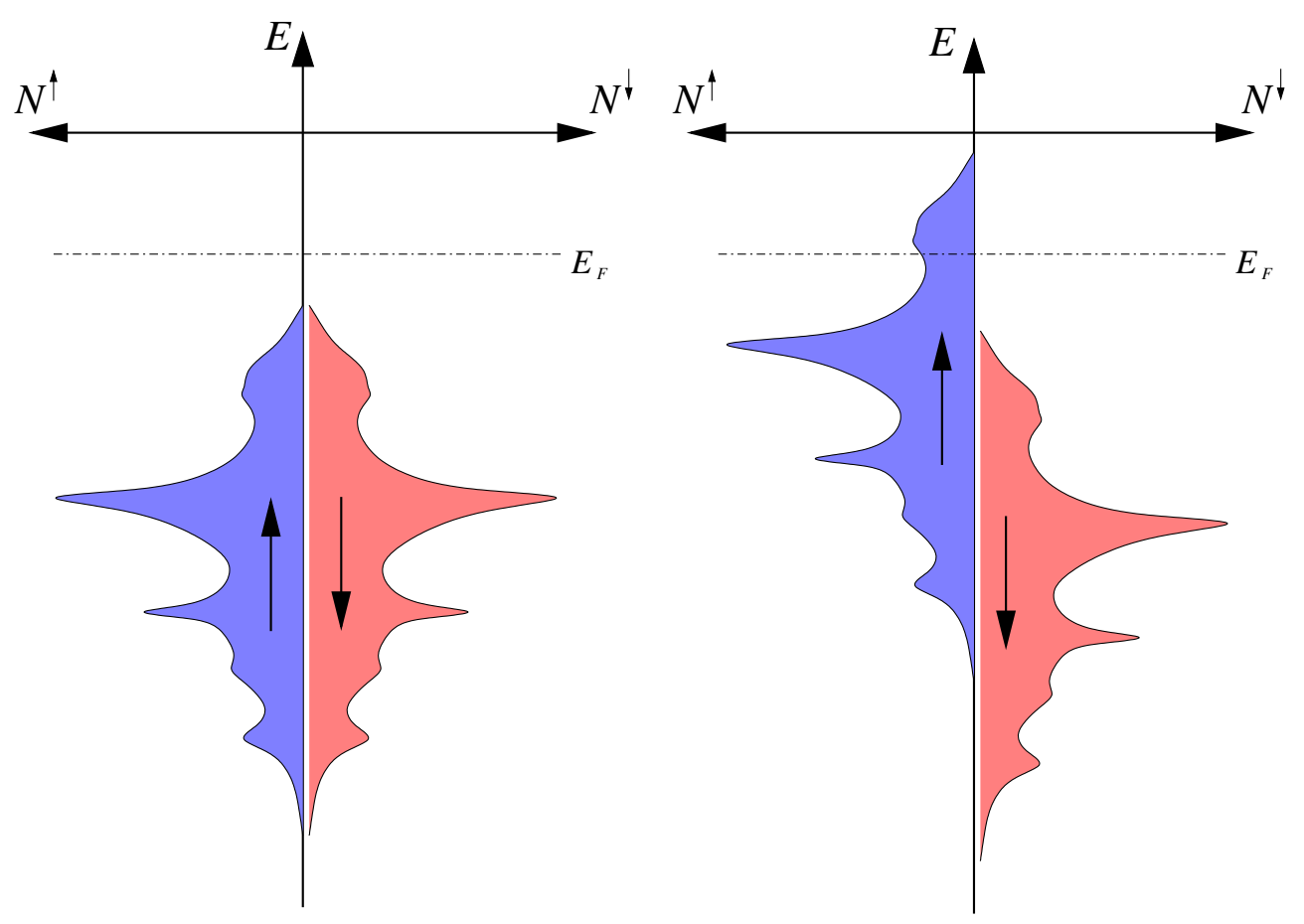

Figure 2.1: Density of states (DOS) in a non-ferromagnetic (left) and in a ferromagnetic material (right). One energy band is splitted into two half bands of opposite spin orientation.

domain. Each domain is separated from another by so called Bloch walls. The development of domains is controlled by the intensity of the fringing field: if the intensity of the fringing field reaches a critical intensity so that a single domain system is energetically unfavorable, a second domain is created in which the magnetization has the opposite direction of the domain before. The reason is the attraction of the north pole of the first domain to the south pole of the second one and vice versa. However, to create a domain boundary, an amount of energy comparable to that of the fringing fields of the involved domains must be spent.

The situation looks a little different if two different ferromagnetic materials are brought in close proximity. These effects are described in the model of RudermanKittel-Kasuya-Yosida (RKKY) . If two ferromagnetic materials could be brought into direct contact, the magnetization in both materials would align parallely and the two materials could be treated as one material. In reality there is always a very thin spacer material dividing the two blocks from each other. This spacer is normally an oxidic interlayer, a contamination or simply roughness, which prohibits a direct contact of the two blocks. In this case, something like a domain boundary is present in terms of solid material. Hence, the direction of the magnetization is aligned antiparallel, as long as no external magnetic field is present (compare to Fig. 2.2). This process is called Interlayer Exchange Coupling (IEC). Experiments have shown that IEC of ferromagnetic materials across inter-layers can be described 


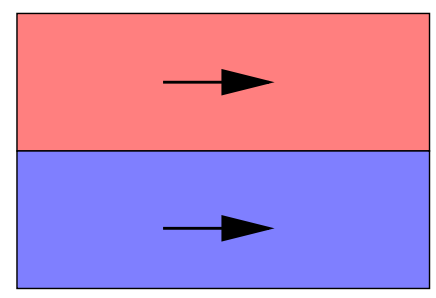

Material A

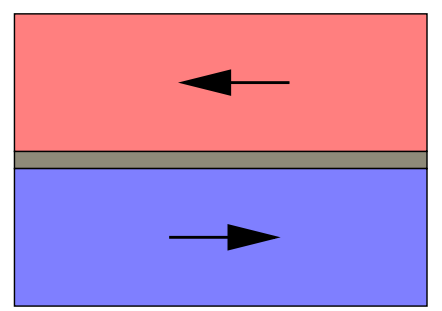

Figure 2.2: Alignment of the magnetization in two ferromagnetic materials brought into close proximity. The left image shows a non-realistic ideal case, where two materials are brought directly together, while the right image includes a possible thin interlayer that has no ferromagnetic properties. For detailed explanation see text.

by an energy density $\sigma_{I E C}$ which is described by the relation

$$
\sigma_{I E C}=-J_{1} \cdot \cos (\vartheta)-J_{2} \cdot \cos ^{2}(\vartheta)
$$

where $J_{1}$ and $J_{2}$ describe the type and the strength of the coupling and $\vartheta$ is the angle between the two directions of magnetization $M_{1}$ and $M_{2}$ of the two ferromagnetic layers. The term including $J_{1}$ describes the bilinear coupling. If this part dominates then, from the minima of Eq. 2.1, the coupling is ferromagnetic (anti-ferromagnetic) for positive (negative) $J_{1} . \quad J_{2}$ represents the biquadratic coupling. This kind of coupling mainly occurs due to interface roughness. If this term dominates and furthermore is negative, $90^{\circ}$-coupling is obtained.

The effect of antiparallel alignment occurs due to polarization of the electron spins inside the non-ferromagnetic interlayer. Right at the edge of the interlayer in the direction of the ferromagnetic material, the spins are aligned in direction of the majority electrons of the ferromagnetic material. Due to scattering effects caused by a difference in the potential height at the interlayer-ferromagnet interface, the interlayer electrons create stationary waves. The intensity of these waves causes an oscillating local magnetization behavior inside the interlayer and holds on only over a certain depth of approximately $1.8 \mathrm{~nm}$. The polarization of the electrons of the interlayer influences the magnetization of the opposite ferromagnetic electrode. If the interlayer has a higher thickness and therefore the distance between the two ferromagnetic layers is higher, the magnetization of the two ferromagnetic layers is completely independent of each other. Hence, the general assumption for the occurrence of IEC is the formation of quantum well states within the non-magnetic interlayer caused by spin-dependent electron reflection at the interface of the ferromagnetic electrode and the non-ferromagnetic interlayer. This so-called Quantum Confinement Model has become the generally accepted model for explaining the IEC. 

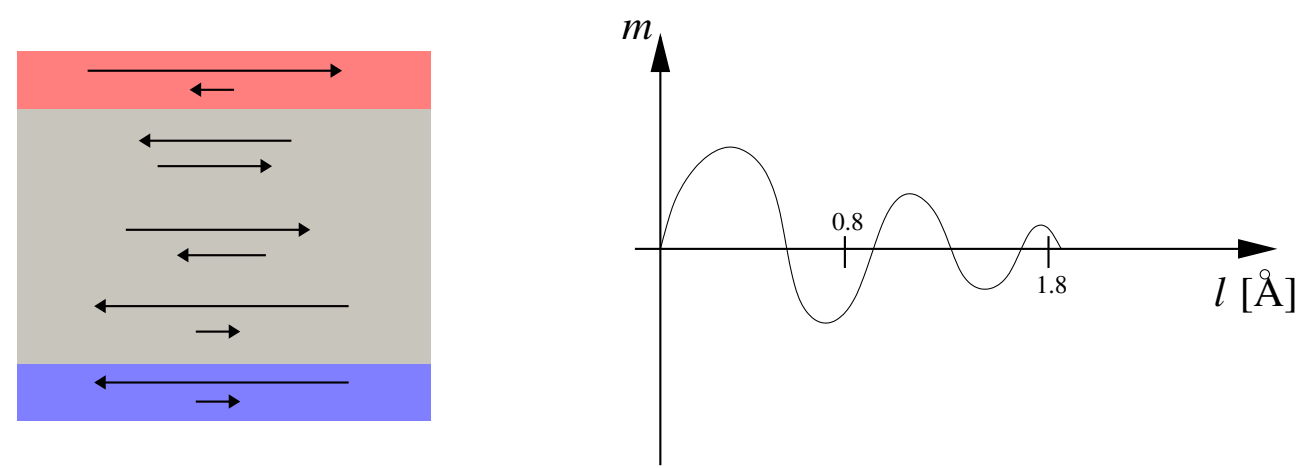

Figure 2.3: Spin polarization inside a non-ferromagnetic interlayer caused by the attached ferromagnet. Left image illustrates the spin alignment of the electrons in the two half-bands, while the right graph shows the oscillation of the magnetization. After a length of $\sim 1.8 \AA$ the magnetization amplitude approaches continously 0 .

\subsubsection{Giant Magneto-Resistance (GMR)}

Adjacent ferromagnetic materials align their magnetization in an antiparallel way because of interlayer exchange coupling (see previous section). In 1986 it was discovered that two ferromagnetic layers separated by a thin $(\sim 1 \ldots 2 \mathrm{~nm})$ nonferromagnetic interlayer also align antiparallel if no external magnetic field is present (Grünberg et al., 1986). If the magnetization is aligned parallely by the application of an external magnetic field, the resistance of the setup decreases. The change of the resistance depends upon the angle between the direction of the magnetization and the current and is a result of spin-dependent scattering of the current electrons. The strength of the GMR effect can be described by

$$
\frac{\Delta R}{R_{P}}=\frac{R_{A P}-R_{P}}{R_{P}}
$$

where $R_{P}$ is the resistance of the parallel alignment and $R_{A P}$ the resistance of the antiparallel alignment, respectively. There are two possible geometries, namely the CIP(current in plane) and CPP (current perpendicular plane).

The mechanism that makes GMR possible can be explained by Mott's two currents model, which assumes two independent current channels for spin-up and spin-down electrons. In a material that is magnetized in parallel, electrons belonging to the spin-up channel show reduced scattering effects, while electrons in the spin-down channel are scattered predominately. If the material is magnetized antiparallel, spin-up electrons as well as spin-down electrons are scattered in the same way, hence the resistance of the device is increased in antiparallel alignment. Hence, in a GMR device, where both layers are magnetized in the same direction, the resistance is lower than in the case that the magnetization of the electrodes is aligned in opposite direction. The sketched principle can be assumed as correctly in the case that the spin of the electron is conserved during the scattering process. 


\subsubsection{Tunnel Magneto-Resistance (TMR)}

A paramagnetic TMR setup is constructed similarly to the GMR setups, except the intermediate layer is replaced by an insulating material, mostly $\mathrm{Al}_{2} \mathrm{O}_{3}$ with a thickness of $1 \ldots 2 \mathrm{~nm}$ located between two ferromagnetic layers . If a voltage is applied to the setup, a relatively small quantum-mechanical tunneling current flows through the barrier. Therefore TMR can only be observed in CPP arrangements.

\section{The Jullière model}

Jullière discovered in 1975 , that the resistance of an element consisting of two ferromagnetic layers separated by a thin insulating barrier vanishes with the application of an outer magnetic field (Jullière, 1975). In those days, it was already known that tunneling current through a thin insulating barrier is possible as described by Sommerfeld and Bethe decades before. Jullière also assumed that the tunneling electrons conserve their spin.

The model of Jullière considers a positive magneto-resistance, i.e. that the spin-up electrons are supposed to be in the majority. At a small bias, most of the electrons tunnel near the Fermi level. The spin polarization $P_{i}$ represents the asymmetry of the spin-up and spin-down electrons at the Fermi energy (compare to Fig. 2.1) and can be written as

$$
P_{i}=\frac{N_{\uparrow}^{E_{F}}-N_{\downarrow}^{E_{F}}}{N_{\uparrow}^{E_{F}}+N_{\downarrow}^{E_{F}}}
$$

where $N_{\uparrow}^{E_{F}}$ represents the number of spin-up electrons and $N_{\downarrow}^{E_{F}}$ the number of spindown electrons at the Fermi level, respectively.

If the electrons are assumed to conserve their spin during tunneling, spin-up and spin-down electrons of $\mathrm{M}_{A}$ tunnel into the empty spin-up and spin-down states of $\mathrm{M}_{B}$, respectively. Assuming the tunneling probability proportional to the product of the DOS of each material $\mathrm{M}_{A}$ and $\mathrm{M}_{B}$, the tunneling conductance $G_{P}$ can be described as

$$
G_{P} \propto N_{\uparrow}^{E_{F}}(1) N_{\uparrow}^{E_{F}}(2)+N_{\downarrow}^{E_{F}}(1) N_{\downarrow}^{E_{F}}(2)
$$

In the case of antiparallel configurations, the roles of majority and minority electrons are switched between $\mathrm{M}_{A}$ and $\mathrm{M}_{B}$. Therefore, spin-up (spin-down) electrons of $\mathrm{M}_{A}$ do not have enough empty spin-up (spin-down) states in $\mathrm{M}_{B}$. As a consequence, the tunneling current through the barrier is reduced and the resistance increases. The conductance in the antiparallel case can be described by

$$
G_{A P} \propto N_{\uparrow}^{E_{F}}(1) N_{\downarrow}^{E_{F}}(2)+N_{\downarrow}^{E_{F}}(1) N_{\uparrow}^{E_{F}}(2) .
$$

The TMR effect, which is defined as the change of conductance $\Delta G$ between the parallel and antiparallel configuration derived by the conductance in the parallel configuration $G_{P}$, can be written as

$$
\frac{\Delta G}{G_{P}}=\frac{2 P_{1} P_{2}}{1+P_{1} P_{2}}
$$


as simply shown by combining Eq. 2.3, Eq. 2.4 and Eq. 2.5. The TMR vanishes if either $P_{1}$ or $P_{2}$ is zero i.e. if one of the materials has no ferromagnetic properties at all. Hence, TMR effects are possible only in the case that both electrodes have ferromagnetic properties.

If the magnetization of the electrodes is neither parallel nor antiparallel but perpendicular - e.g. if $\mathrm{M}_{A}$ is assumed to be magnetized with spin-up and spin-down electrons and $M_{B}$ with spin-left and spin-right electrons. Neither spin-left nor spinright are eigenvalues of the wave function of the spin in $\mathrm{M}_{A}$, but they are a linear combination of those. As a consequence, the spin-up electrons of $\mathrm{M}_{A}$ have the same probability to enter spin-right or spin-left states. Taking all these aspects into account, the conductance due to spin-up and spin-down electrons from $\mathrm{M}_{A}$ can be written as

$$
\begin{aligned}
G_{\uparrow} & \propto \frac{1}{2} N_{\uparrow}^{E_{F}}(1) N_{\leftarrow}^{E_{F}}(2)+\frac{1}{2} N_{\uparrow}^{E_{F}}(1) N_{\rightarrow}^{E_{F}}(2) \\
G_{\downarrow} & \propto \frac{1}{2} N_{\downarrow}^{E_{F}}(1) N_{\leftarrow}^{E_{F}}(2)+\frac{1}{2} N_{\downarrow}^{E_{F}}(1) N_{\rightarrow}^{E_{F}}(2)
\end{aligned}
$$

The total conduction in the TMR setup is the sum of both. It is obviously clear that it is the average value of the tunneling conductance for the parallel and antiparallel alignment.

\section{The Slonczewski model}

14 years after the model by Jullière, Slonczewski gave a quantitative model to calculate spin-dependent tunneling of a free electron including spin and magnetic properties (Slonczewski, 1989). The magnetization $m$ of the two electrodes $\mathbf{M}_{A}$ and $\mathbf{M}_{B}$ separated by a thin insulating barrier are assumed to be arbitrary. As shown in Fig. 2.4. Slonczewski assumed two potentials $U$ (regions 1 and 3) separated by a finite insulating rectangular barrier with a potential $U_{0}$ and a vanishing molecular field $h=0$ (region 2). To simplify the model, Slonczewski assumed the external voltage between the electrodes $V=0$ and $\left|h_{A}\right|=\left|h_{B}\right|=h_{0}$. The values of $h_{A}$ and $h_{B}$ as well as the spin quantization axes $z$ and $z^{\prime}$ differ by an angle of $\Theta$. In a free electron model of the spin-polarized conduction, electrons inside each electrode (regions 1 and 3) have the effective Hamiltonian for one electron, that can be described with the following Schrödinger equation

$$
H_{\xi}=-\frac{1}{2}\left(\frac{d}{d \xi}\right)^{2}+U(\xi)-h(\xi) \cdot \sigma
$$

where $-h(\xi) \cdot \sigma$ represents the internal exchange energy, $-h(\xi)$ the molecular field and $\sigma=2 s$ the Pauli spin operator ${ }^{2}$. From the Schrödinger equation (2.9) the energy for the free electron inside one of the ferromagnetic electrodes can be calculated by:

$$
E_{\xi}=\frac{1}{2} k_{\sigma}^{2}-\sigma h_{0}, \quad \sigma= \pm 1
$$

\footnotetext{
${ }^{2} s$ is the eigenfunction of $\operatorname{spin} \pm \frac{1}{2}$
} 


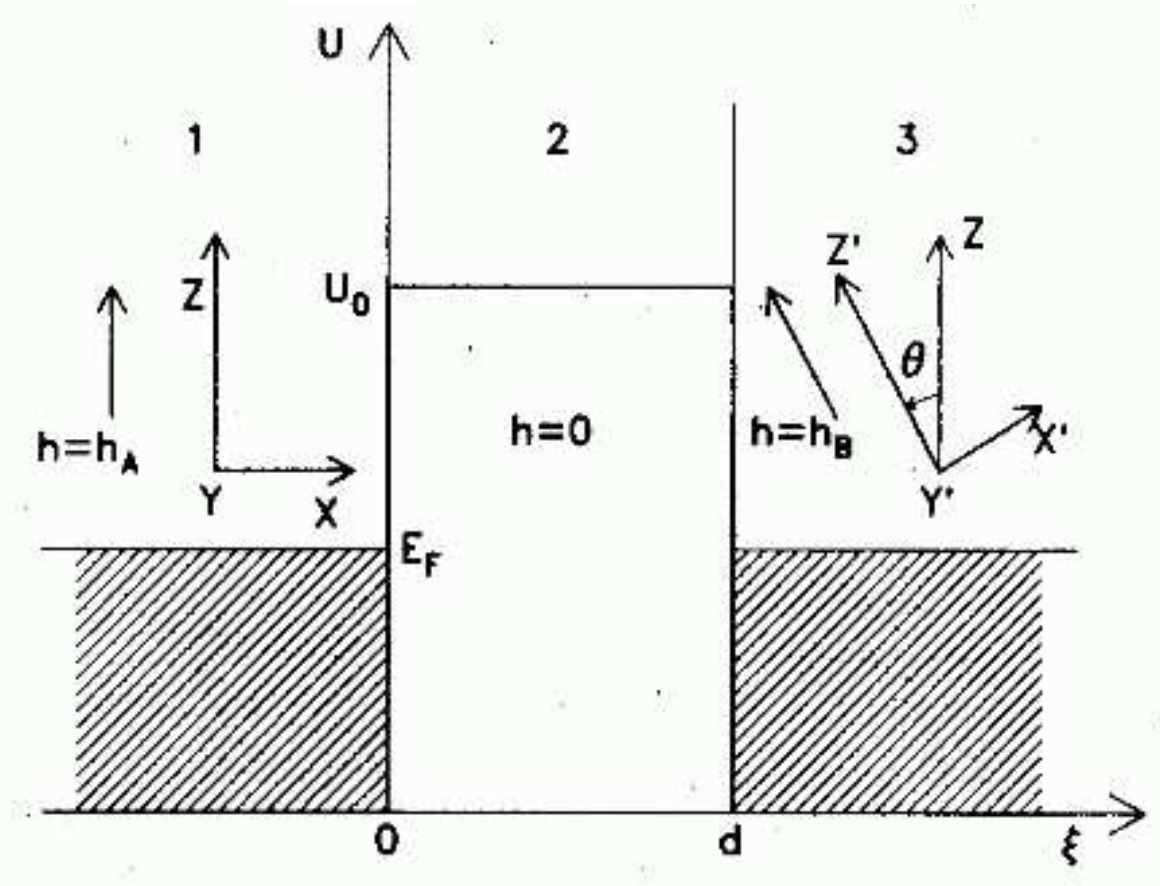

Figure 2.4: Schematic diagram of the conditions assumed in the model by Slonczewski. For each potential an own Hamiltonian is derived including magnetic and spin properties (Slonczewski, 1989).

where $k_{\sigma}$ represents the electron momenta for spin-up and spin-down electrons. Inside the insulating barrier the energy can be written as

$$
E_{\xi}=\frac{1}{2} \kappa^{2}+U_{0}, \quad \sigma=\uparrow, \downarrow
$$

where $i \kappa$ is the imaginary momentum inside the barrier. If a spin-up incident plane wave with unit incident flux in region 1 is considered, (ferromagnetic electrode, $\xi<0$ in Fig 2.4 and all the boundary conditions between the potentials are taken into account, the eigenfunctions of $H_{\xi}$ (eigenvalue $E_{\xi}$ ) in the three regions can be derived:

In region $1(\xi<0)$ :

$$
\psi_{\uparrow 1}=k_{\uparrow}^{-1 / 2} e^{i k_{\uparrow} \xi}+R_{\uparrow} e^{-i k_{\uparrow} \xi}, \quad \psi_{\downarrow 1}=R_{\downarrow} e^{-i k_{\downarrow} \xi}
$$

In region $2(0 \leq \xi \leq d)$ :

$$
\psi_{\sigma 2}=A_{\sigma} e^{-\kappa \xi}+B_{\sigma} e^{\kappa \xi}, \quad \sigma=\uparrow, \downarrow
$$

In region $3(\xi<d)$ :

$$
\psi_{\sigma 3}=C_{\sigma} e^{i k_{\sigma}(\xi-d)}, \quad \sigma=\uparrow, \downarrow
$$

In order to obtain the unknown variables $R_{\sigma}, A_{\sigma}, B_{\sigma}, C_{\sigma}(\sigma=\uparrow, \downarrow), \psi_{\sigma}$ and $d \psi_{\sigma} / d \xi$ have to be matched at the interfaces $\xi=0$ and $\xi=d$. With these derived parameters 
the transmission coefficient $T$ through the barrier can be calculated:

$$
\begin{aligned}
T_{z} & =\operatorname{Im} \sum_{\sigma} \sigma \psi_{\sigma}^{*}\left(\frac{d \psi_{\sigma}}{d \xi}\right) \\
T_{x}+i T_{y} & =i\left(\frac{d \psi_{\uparrow}^{*}}{d \xi} \psi_{\downarrow}-\psi_{\uparrow}^{*} \frac{d \psi_{\downarrow}}{d \xi}\right),
\end{aligned}
$$

where $T=T_{x}+T_{y}+T_{z}$ is the expectation value of the Pauli spin $(\sigma=2 s)$ transmitted through the area with given $\xi$. The expression in Eq. 2.15 for $T_{z}$ becomes the conventional particle transmissivity $T_{p}$ when the factor $\sigma$ in the summand is removed:

$$
T_{p}=\operatorname{Im} \sum_{\sigma} \psi_{\sigma}^{*}\left(\frac{d \psi_{\sigma}}{d \xi}\right)
$$

Considering nearly zero temperature and taking into account the limit of a small barrier factor, a narrow distribution of electrons near the normal incidence with $E_{\xi}$ close to $E_{F}$ is responsible for most of the current. Therefore, $\kappa\left(E_{\xi}\right)$ and $k_{\sigma}\left(E_{\xi}\right)$ can be replaced by $\kappa\left(E_{F}\right)$ and $k_{\sigma}\left(E_{F}\right)$ in calculating the conductance $G$ due to tunneling. Summing over $E_{\xi}$ and $k_{\|}$for occupied states delivers the conventional expression for the tunneling conductance:

$$
G=\left(\frac{e^{2}}{8 \pi^{2} \hbar}\right) \cdot\left(\frac{\kappa T}{d}\right)
$$

For a better understanding, in the next step only TMR effects between two identical magnetic layers $f$ across the barrier $b$ are considered. Including the consideration about transmissivity above, the tunneling conductance can be described by

$$
G=G_{f b f}^{0}\left(1+P_{f b} P_{f b} \cos \theta\right)
$$

with the effective spin polarization

$$
P_{f b}=\frac{\left(K_{\uparrow}-k_{\downarrow}\right)}{\left(K_{\uparrow}+k_{\downarrow}\right)} \cdot \frac{\left(\kappa^{2}-k_{\uparrow} k_{\downarrow}\right)}{\left(\kappa^{2}+k_{\uparrow} k_{\downarrow}\right)}
$$

and the mean conductance

$$
G_{f b f}^{0}=\frac{\kappa}{\hbar d} \cdot\left[\frac{e \kappa\left(\kappa^{2}+k_{\uparrow} k_{\downarrow}\right)\left(k_{\uparrow}+k_{\downarrow}\right)}{\pi\left(\kappa^{2}+k_{\uparrow}^{2}\right)\left(\kappa^{2}+k_{\downarrow}^{2}\right)}\right]^{2} \cdot e^{-2 \kappa d}
$$

In a real application the two electrodes $f$ and $f^{\prime}$ may have different properties and different compositions, and the quantities $k_{\uparrow}$ and $k_{\downarrow}$ have to be assumed as different for $f$ and $f^{\prime}$. Therefore the conductance can be written in a more general manner:

$$
G=G_{f b f^{\prime}}^{0}\left(1+P_{f b} P_{f^{\prime} b} \cos \theta\right)
$$

where the spin polarizations are given by

$$
\begin{aligned}
P_{f b} & =\frac{\left(K_{\uparrow f}-k_{\downarrow f}\right)}{\left(K_{\uparrow f}+k_{\downarrow f}\right)} \cdot \frac{\left(\kappa^{2}-k_{\uparrow f} k_{\downarrow f}\right)}{\left(\kappa^{2}+k_{\uparrow f} k_{\downarrow f}\right)} \\
P_{f^{\prime} b} & =\frac{\left(K_{\uparrow f^{\prime}}-k_{\downarrow f^{\prime}}\right)}{\left(K_{\uparrow f^{\prime}}+k_{\downarrow f^{\prime}}\right)} \cdot \frac{\left(\kappa^{2}-k_{\uparrow f^{\prime}} k_{\downarrow f^{\prime}}\right)}{\left(\kappa^{2}+k_{\uparrow f^{\prime}} k_{\downarrow f^{\prime}}\right)} .
\end{aligned}
$$




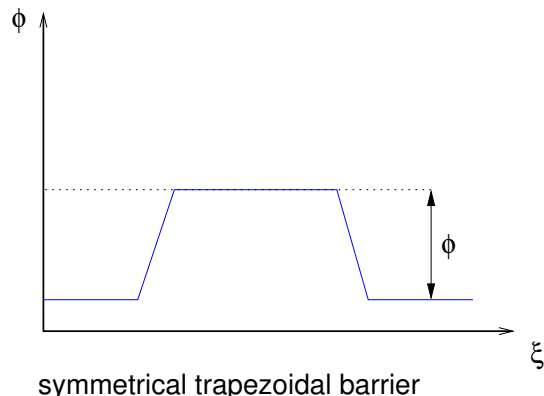

Figure 2.5: Barriers assumed in the Brinkman model for a trapezoidal barrier.

\section{Jullière and Slonczewski; a final conclusion}

Compared to the model by Jullière, the model by Slonczewski is a completely different approach to the theory of tunnel magneto-resistance. The Jullière model describes the effects in a phenomenological way using the model of band-structures and polarization of electrons, while the approach by Slonczewski uses the theory of quantum mechanics including magnetic properties. MacLaren et al. (1997) compared the models by Jullière and Slonczewski. They pointed out, that both models do not deliver a reasonable explanation for spin-dependent tunneling. Although Slonczewski gives a reasonable approximation for free electrons, it is not clear how his theory can be applied to materials with more complicated band structures. The theory by Jullière does not include any specific material properties. Additonaly Tsymbal and Pettifor (1998) have shown that the Jullière model agrees well with the magneto-resistance for high disorder in the barrier.

Finally, one can determine that a successful model needs to include the complete band structure in presence of the interfaces as well as the dependence of the tunneling current including parameters like barrier hight and thickness. But until now, no such all-including model is available.

\subsection{Derivation of barrier properties}

In the early sixties Simmons (1963) derived a theoretical model for calculation of properties of insulating barriers like barrier height and barrier width aligned within two conductive layers using the conductivity that can be derived by the $U / I$ characteristic curve. Seven years later, this model was further developed by Brinkman et al. (1970).

\section{The Brinkman model}

Brinkman et al. (1970) calculated the barrier conductance assuming two different kinds of models for the barrier. In one model, the barrier is assumed to have a 
trapezoidal barrier structure while in the other one, the boundaries between the metals and the barrier are assumed to be sharp. For the first model the barrier conductance is calculated by applying the $\mathrm{WKB}^{3}$ model. The tunneling current is integrated in all three dimensions, given a height of the barrier $\bar{\varphi}$, the barrier thickness $d$ and the asymmetry $\Delta \varphi$, the results are shown in Fig. 2.6. In each plot two of the three parameters $(d, \bar{\varphi}, \Delta \varphi)$ are fixed. Finally the conductance is calculated by fitting a second order polynomial to the values for the conductance as a function of the bias voltage. According to the mathematical model one should expect the curves to show a parabolic and a symmetrical behavior around the minimum. In fact the plots shown in Fig. 2.6 do not show such a behavior. For example, the plot in the bottom of Fig. 2.6 deviates by $4 \%$ from parabolic behavior at $200 \mathrm{mV}$ (Brinkman et al., 1970). This problem can be solved by including polynomials of higher order. For low voltages $(e U<<\bar{\varphi})$ the differential conductivity can be described by a second order polynomial, where the constants $A, B$ and $C$ represent a function of the barrier parameters:

$$
G=\frac{d I_{\text {total }}}{d U}=A(\bar{\varphi}, d) U^{2}+B(\bar{\varphi}, \Delta \varphi) U+C(\bar{\varphi}, d)
$$

With Eq. 2.25 the constants $A, B$ and $C$ can be determined from experimental $U / I$ characteristic curves and from these, equations the required parameters can be derived (Schmalhorst, 2001):

$$
\begin{aligned}
\varphi^{2} & =\frac{e^{2} C}{32 A}\left[\ln \left(\frac{h^{3}}{\sqrt{2} \pi e^{3} m_{e f f}} \cdot \sqrt{A C}\right)\right]^{2} \\
d & =-\frac{\hbar}{8 \sqrt{\varphi m_{e f f}}} \cdot \ln \left(\frac{\hbar^{3}}{\sqrt{2} \pi e^{3} m_{e f f}}\right) \\
\Delta \varphi & =-\frac{12 \hbar \bar{\varphi}^{\frac{2}{3}} B}{\sqrt{2 m_{e f f}} e d C}
\end{aligned}
$$

where $m_{\text {eff }}$ is the effective mass of the electrons. For $\mathrm{Al}_{2} \mathrm{O}_{3}$, this value can be assumed to be $0.3 \cdot m_{e}$ (Bratovsky, 1997).

From the measurement of an $U / I$ characteristic curve and the fit of a second order polynomial after Eq. 2.25) to the differential conductivity derived by the $U / I$ measurement, the barrier properties can be calculated by using Eq. 2.26) to (2.28).

In the second model, the barrier is assumed to have sharp boundaries to the surrounding metal layers. In this case the tunneling currents depend on the Fermi energies and also on the DOS of the metals. This model also produces a general parabolic behavior as in the model discussed before, but there are other significant differences between the curves assuming a trapezoidal barrier and a barrier with sharp boundaries.

In reality, the interface between the barrier and the metal layers is never really sharp. It is always contaminated with oxide material or influenced by surface roughness. Hence the model assuming the trapezoidal barrier structure was used in this work to determine barrier properties from the electrical $U / I$ characteristics.

\footnotetext{
${ }^{3}$ Wenzel-Kramer-Brillouin Model
} 

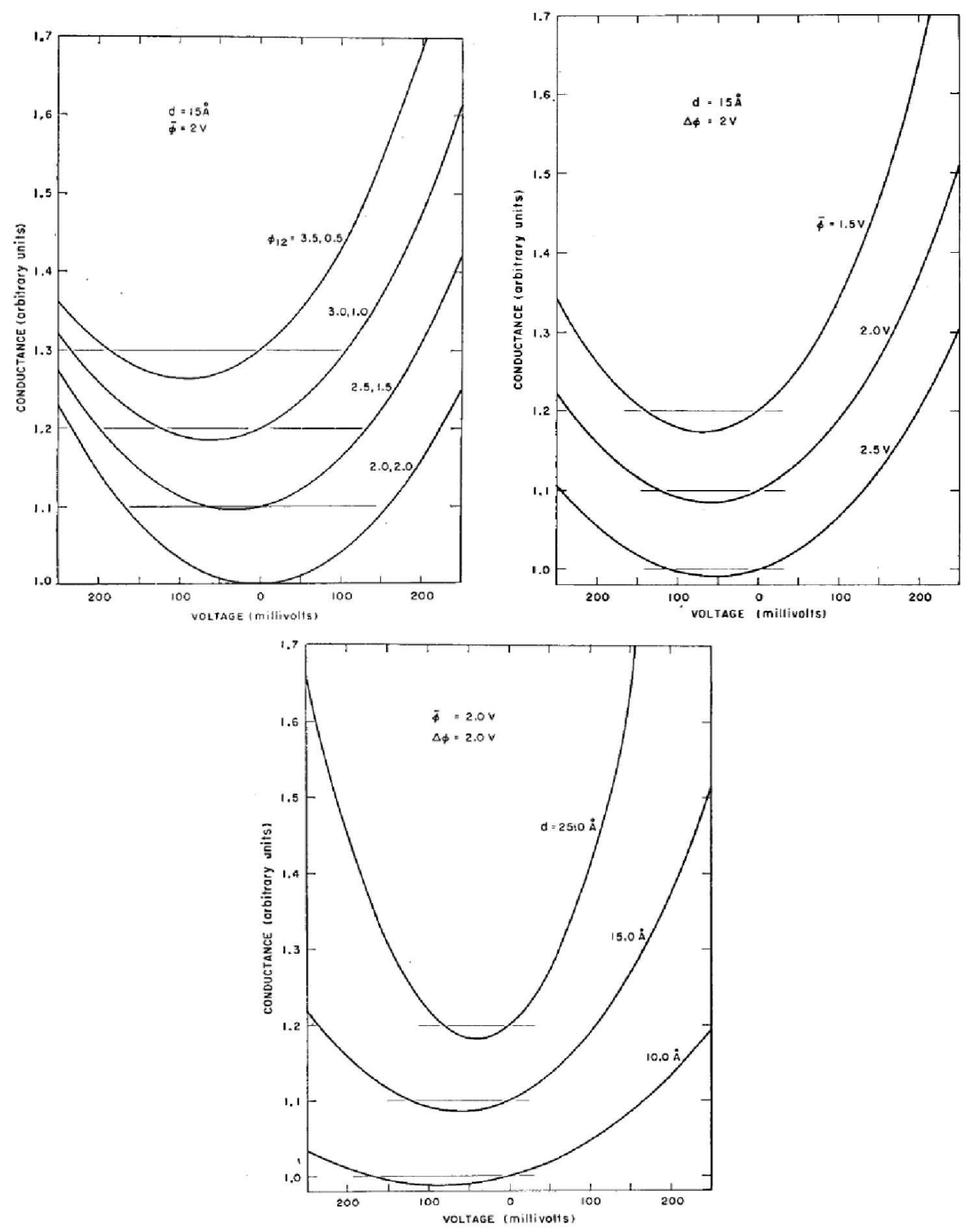

Figure 2.6: Fitted curves for the barrier conductance assuming a trapezoidal barrier. The fixed values for each plot are given above the curves. The conductances are normalized to 1.0 at zero voltage (Brinkman et al., 1970). 
THEORETICAL BACKGROUND

\subsection{Investigations of tunnel barrier structures}

Although the TMR phenomenon has been known since the experiments by Jullière in 1975, the interest for these devices was not very high and only a modest number of investigations have been done until the mid-1990s. The reason is that only low TMR values have been achieved in most experiments. Furthermore, the fabrication of such thin and pinhole-free oxide barriers seemed to be quiet complicated. The situation changed after experiments performed by Miyazaki and Tezuka in 1995 showed higher TMR values in $\mathrm{Fe} / \mathrm{Al}_{2} \mathrm{O}_{3} / \mathrm{Fe}$ junctions, and Moodera et al. (1995) investigated new fabrication technologies which made thin pinhole-free oxide barriers possible.

Oxide tunnel barriers are currently of interest in TMR devices, for use in non volatile storage and sensor applications (Meservey et al. (1970), Slonczewski (1989), Moodera et al. (1995)). TMR devices deliver a base resistance more than two magnitudes higher than those based on the giant magneto resistance (GMR) effect and are therefore potentially usable in CPP arrangements (Parkin et al., 1999b). They typically consist of an oxide barrier with a thickness of $1-2 \mathrm{~nm}$ embedded between two ferromagnetic electrodes of different coercitivity. By changing the relative orientation of the magnetizations of the electrodes, one can vary the spin dependent current through the barrier and by that the resistance of the device by several tens of percent. These so-called spin-valve setups could be organized in arrays, in which the bottom and the top electrode are contacted to word and bit lines in order to change the orientation of the magnetization of the soft magnetic component and to read out the status of a given memory element.

For any technical application, a sufficient thermal and electrical stability is required as these parameters have a direct influence on the lifetime. Therefore, it is important to study the structural modification of the oxide barrier after thermal or electrical stress. Experiments have shown the TMR effect to depend on annealing treatments subsequent to layer deposition. Sousa et al. (1999) e.g. have presented data for heat treatments at temperatures ranging from $30^{\circ} \mathrm{C}$ up to $260^{\circ} \mathrm{C}$. Remarkably, the TMR at first increases with annealing, revealing a maximum after annealing at $210^{\circ} \mathrm{C}$. Schmalhorst et al. (2000) have investigated the voltage stability of $\mathrm{Co} / \mathrm{Al}_{2} \mathrm{O}_{3} / \mathrm{Co}$ setups as a function of the annealing temperature. Samples in as-prepared state show typical breakthrough voltages between 1.3 and $1.5 \mathrm{~V}$. The breakthrough itself happens in a single discontinuous fatal increase of the current. Annealed samples $\left(T=380^{\circ} \mathrm{C}\right)$ show a very different behavior: their breakthrough voltage has decreased to $\sim 0.9 \mathrm{~V}$ and damage appears with a sequence of steps in a quasi-continuous manner.

In order to understand these processes, one needs to investigate the internal chemical and physical structure of the oxide barriers at nearly atomic resolutions. A question of primary importance is the homogeneity in thickness. Another question is the distribution of oxygen inside the spin-valve setup. The schematics in Fig. 2.9 illustrates four possible situations which might be realized: in the ideal case (a), distinguished by a rectangular composition profile, the oxygen is located exactly inside the barrier and the stoichiometry of $\mathrm{Al}_{3} \mathrm{O}_{2}$ is fulfilled (thick solid line). Other 


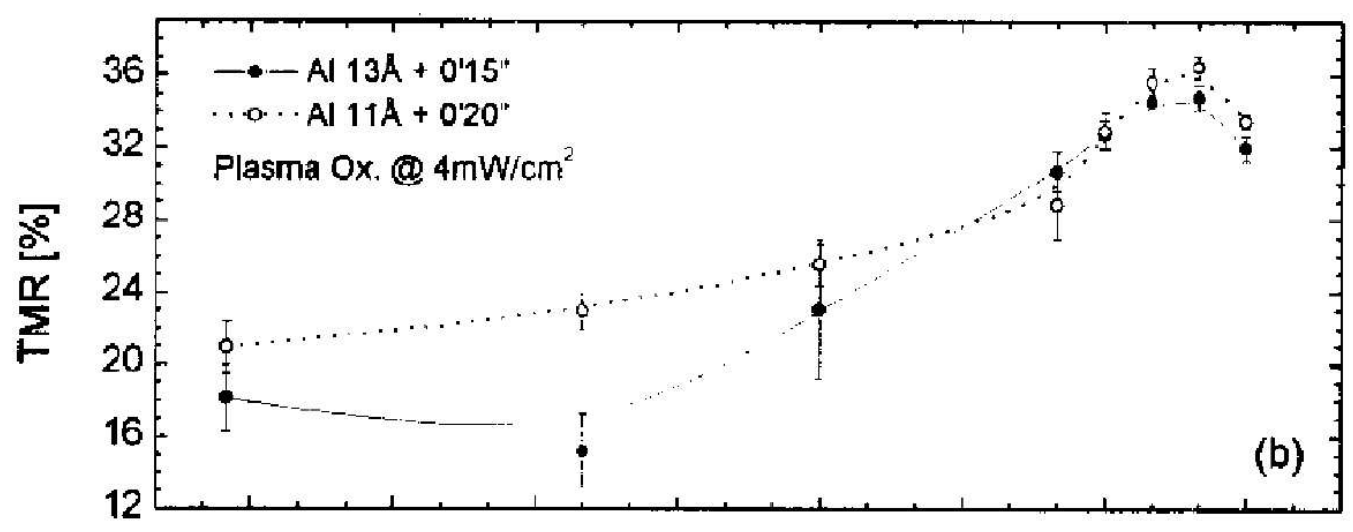

Figure 2.7: Thermal treatment of TMR setups with different barrier thicknesses. Both regimes show a TMR maximum at $\sim 210^{\circ} \mathrm{C}$ (Sousa et al., 1999).

possibilities, which might be the more realistic ones, are an under-abundance (b) or overabundance (c) of oxygen, finally interfacial segregation of oxygen may occur (d).

To optimize potential spin valve structures, it is very important to distinguish among these situations and to clarify the detailed distribution of oxygen and the metal species and its variation after different treatments. In this work TMR spin valve structures are investigated using the 3D tomographic atom probe (TAP). For revealing detailed chemical information, the use of a TAP is expected to be a very suitable method (see section 3.3.2). This all the more as it delivers real 3D data, which may get important to detect local barrier breakthroughs and to characterize interfacial roughness.

The influence of oxygen impurities on the growth of GMR devices has been investigated in a recent study by atom probe tomography (Larson et al., 2003). Since the oxygen content was always below 1 at $\%$, the investigated specimens were completely metallic, ensuring sufficient conductivity to perform field ion microscopy. In contrast, the oxide barriers of interest here, are per definition made of a non-conductive material. Following a general rule of field ion microscopy, this should prevent one from obtaining reasonable measurements. However, in contradiction to this general statement, it will be shown that a real measurement is possible if the thickness of the insulating barrier is only 1 to $2 \mathrm{~nm}$.

Different deposition methods may be applied to produce oxide barriers and the related ferro-magnetic electrodes. For mass production, metallic thin films are usually deposited by magnetron sputtering. The required oxide is then obtained by subsequent plasma oxidation on a previously sputtered Al layer, due to the deposition and implantation of dissociated low energy $(30-80 \mathrm{eV}$ ) oxide ions (see e.g. Moodera et al. (1998), Roos et al. (2001)). Aluminum oxide barriers with a thickness varying from 0.5 to $2 \mathrm{~nm}$ depending on the initial ion energy, are obtained on planar substrates. Unfortunately this method can not be applied to tip-shaped field-ion microscopy samples due to plasma charging effects at the tip apex. Alternative procedures are 


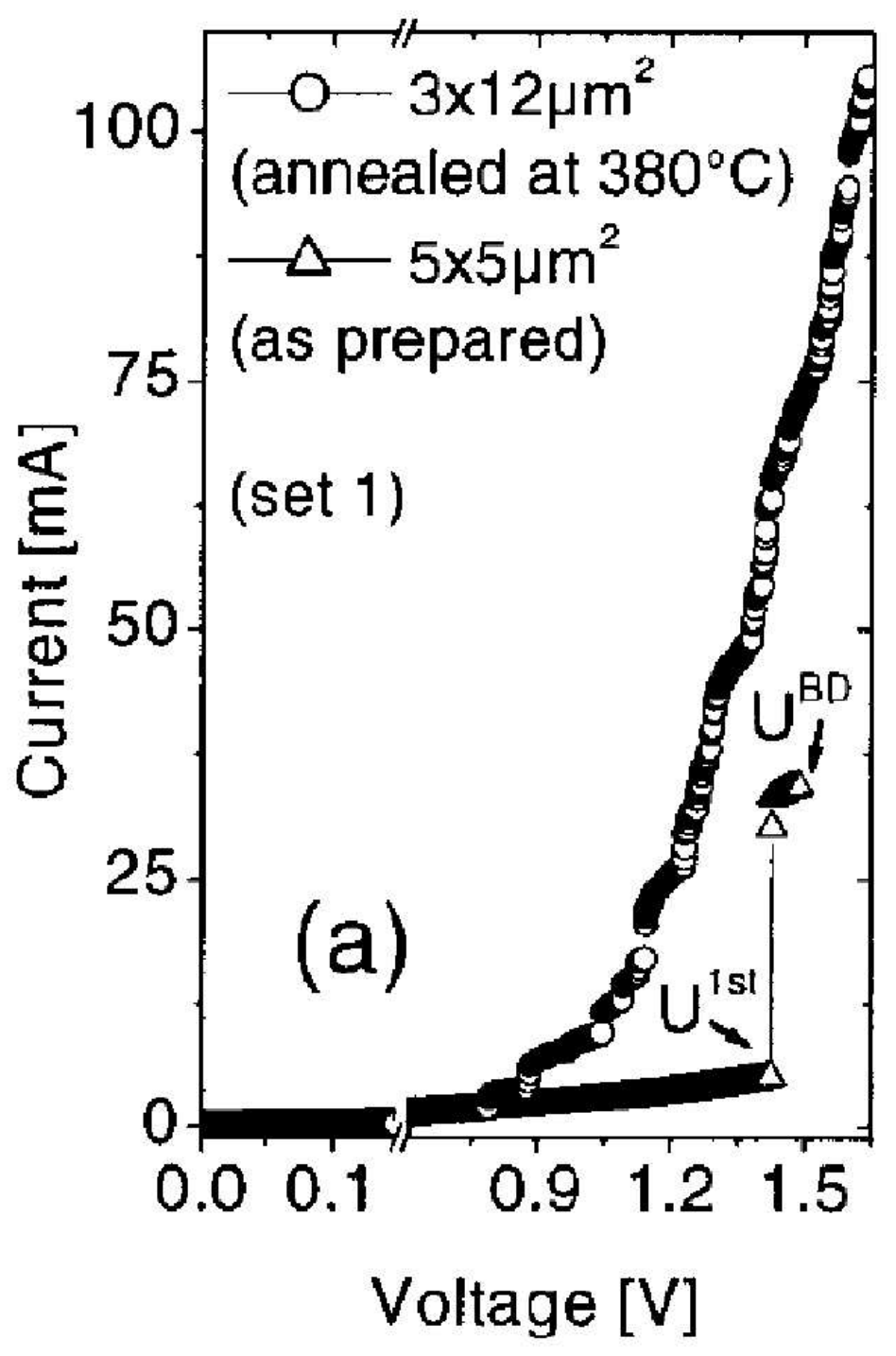

Figure 2.8: Breakthrough voltages for two different sample treatments. One is in as-prepared state while the other was annealed at $380^{\circ} \mathrm{C}$ for $60 \mathrm{~min}$. (Schmalhorst et al. 2000). 


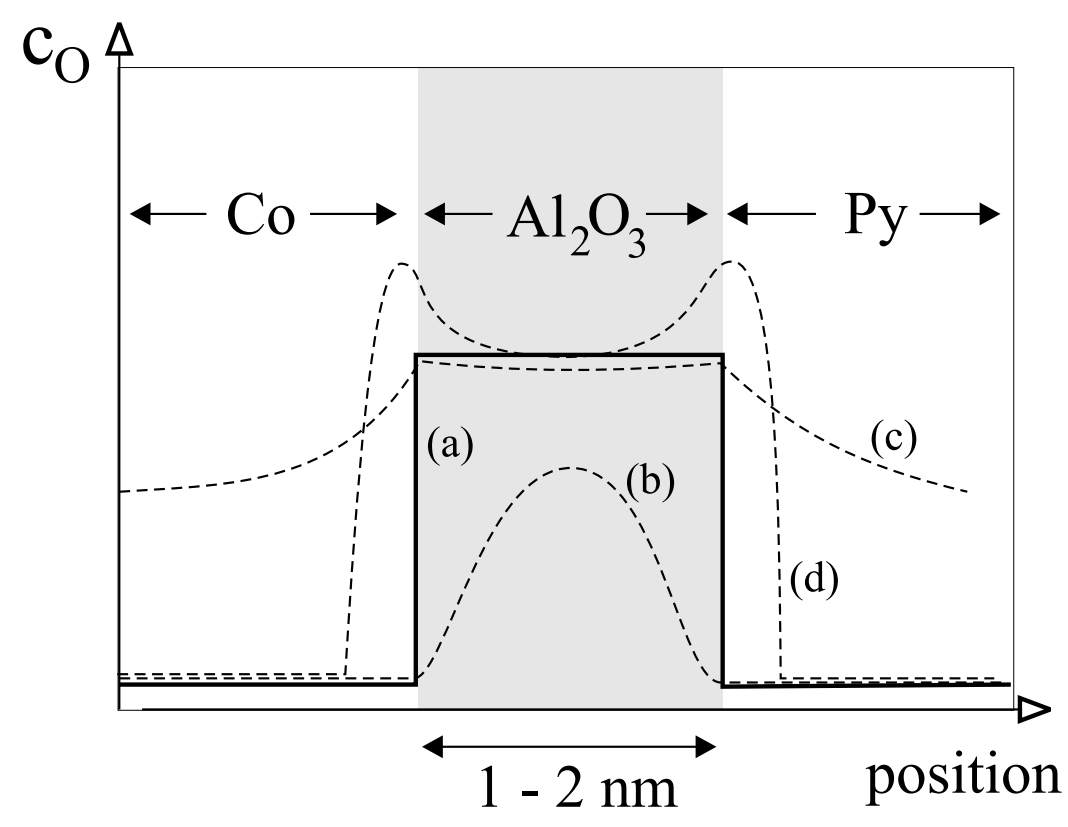

Figure 2.9: Possible distribution of oxygen around the barrier. Solid line (a): ideal distribution. Dashed lines: (b) oxygen under-abundance, (c) oxygen overabundance and (d) segregation at the interfaces.

ozone-assisted oxidation (Park et al. 2002) as well as natural thermal oxidation performed in a stepwise process to obtain sufficient barrier thicknesses (Moon et al. 2002).

In contrast to conventional practices, the model specimens for this investigation have been prepared in an ion beam sputter chamber dedicated to the preparation of thin film specimens for field ion microscopy. Preparation parameters must be optimized to obtain a real insulating barrier and sufficiently stable specimens, able to withstand the considerable field-induced stress during the measurement. The details of preparation will be discussed in the next section. 


\section{Chapter 3}

\section{Experimental methods}

\subsection{Sample preparation}

\subsubsection{Samples for FIM- and TAP-applications}

The layers to be investigated must be deposited on a substrate suitable for measurements by analytical field ion microscopy. At first a $0.1 \mathrm{~mm}$ thick Tungsten wire substrate is thinned by electro-polishing. For that, the wire (with an approximate length of $1.5 \mathrm{~cm}$ ) is first crimped in a copper tube and afterwards inserted into $2 \mathrm{~mol}$ $\mathrm{NaOH}$ solution. Using an $\mathrm{AC}$ voltage of $8 \ldots 12 \mathrm{~V}$ the wire is then thinned to a very low tip radius of approximately $20 \ldots 40 \mathrm{~nm}$.

After electro-polishing, the specimens are inserted into a standard field ion microscope, where the tip voltage is continously raised up to $14 \mathrm{kV}$ in order to create specimen with a reproducible tip radius of approximately $30 \mathrm{~nm}$. These tips are finally coated with a TMR spin-valve stack using ion beam deposition (see section 3.2.

\subsubsection{Planar samples}

In addition specimens were characterized by Transmission-Electron-Microscopy (TEM), Secondary-Ion-Mass-Spectrometry (SIMS) and electrical contact experiments. For these techniques, spin valve structures were deposited on planar substrates.

\section{Specimen for TEM-analysis}

For Transmission Electron Microscopy (TEM) analysis the layers to be investigated are deposited on a glass substrate using ion beam deposition (see section 3.2). As substrate, glass object carriers for conventional optical microscopy are used. The layers are deposited under the same parameters as the tip-like samples for TAP analysis in order to make an accurate comparison. 


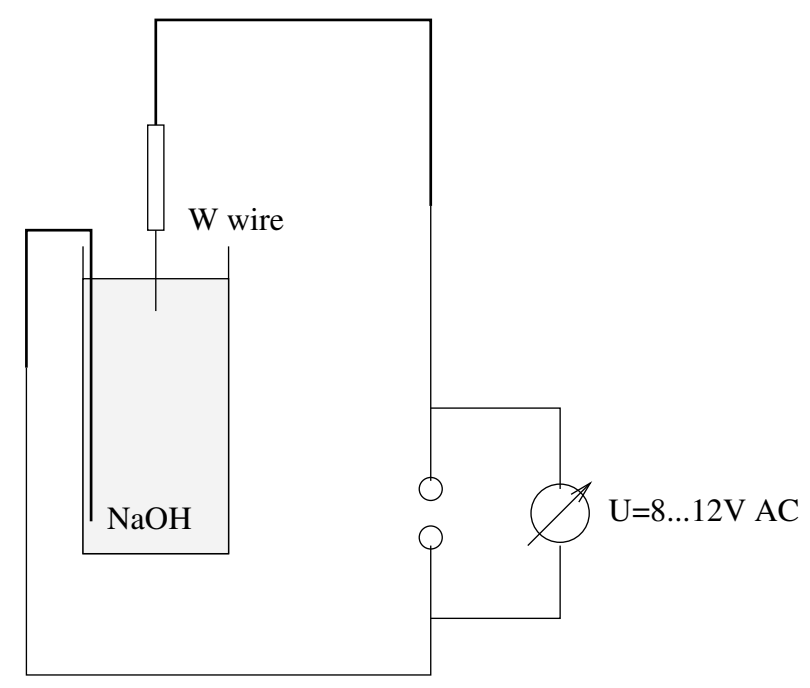

Figure 3.1: Schematic drawing of the electro-polishing process.

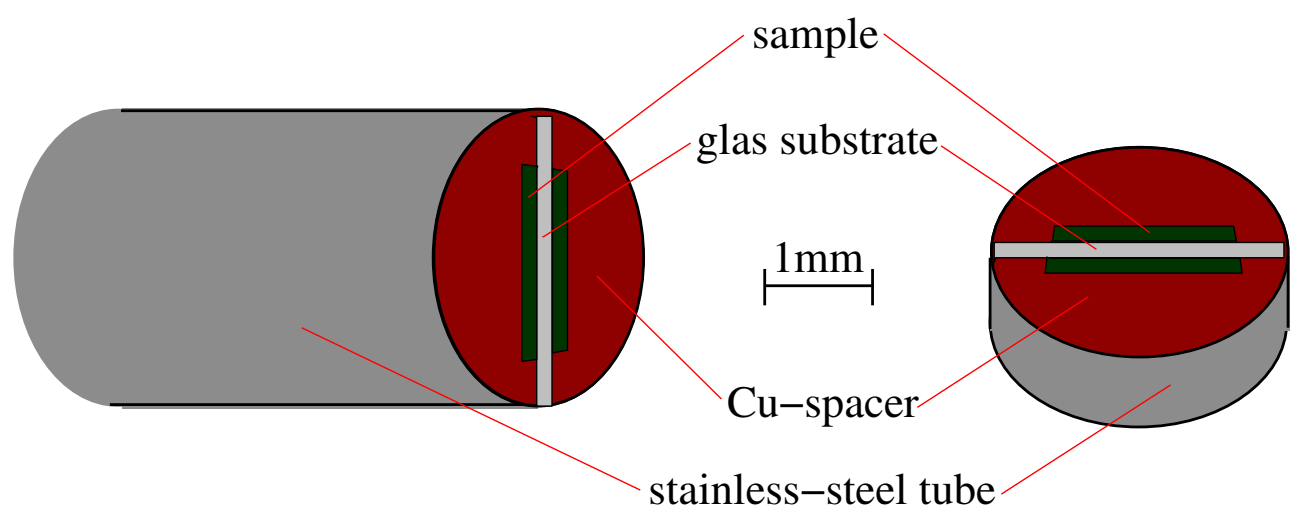

Figure 3.2: Schematic drawing of a prepared sample suitable for TEM analysis. The left figure shows the whole tube, while the right figure illustrates a $0.5 \mathrm{~mm}$ thick disc cut normal to the tube axis. One tube delivers up to 5 useful samples.

The preparation was made using standard procedures for cross-section sample preparation. The substrate with the deposited layers is fitted between two halves of a Copper cylinder into a tiny tube with $\sim 2.3 \mathrm{~mm}$ in diameter using a special glue, to fix the components together. From that tube, small slices of $0.5 \mathrm{~mm}$ in thickness are cut away and subsequently ground manually to a thickness of $\sim 100 \mu \mathrm{m}$. Using dimple grinding the sample is further thinned to a thickness of $\sim 30 \mu \mathrm{m}$. Dimple grinding provides a smooth thinned surface in the center of the specimen. This area of the sample must then be finally thinned to a thickness of $\sim 50 \mathrm{~nm}$, to obtain electron-beam transparency. This is achieved by an ion-polishing device, in which two ion guns alternately emit an Argon ion beam with energies of $2.5-6.0 \mathrm{keV}$ and a low angle to the sample's surface of $2.0-4.5^{\circ}$. 


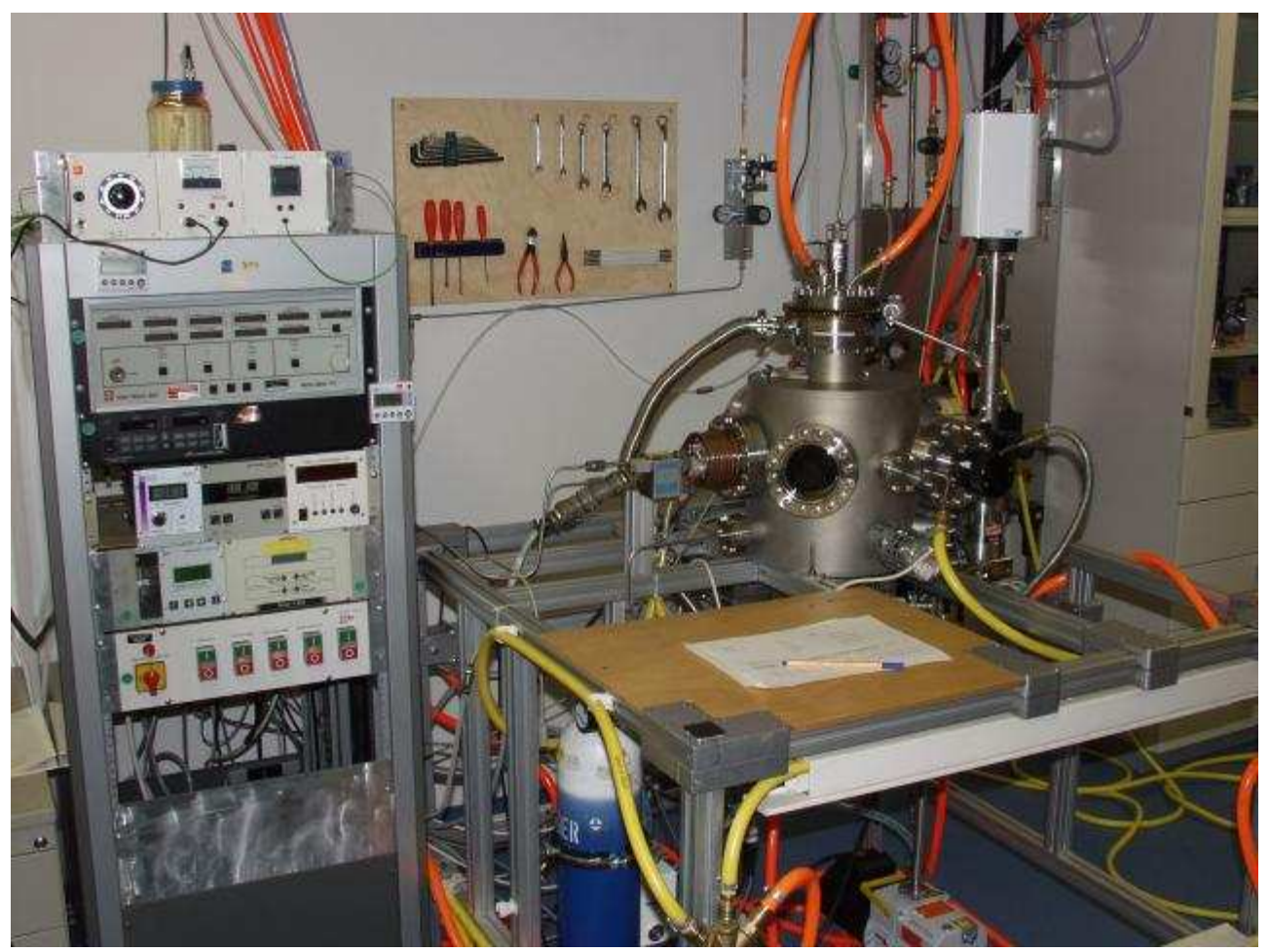

Figure 3.3: The ion beam deposition device at the Institute for Material Physics of the University of Göttingen. The system runs with two separate pumping sections: the smaller one for the mass-spectrometer (white box in the right top); and the larger pumping system providing UHV conditions within a half of a day.

\section{Specimen for SIMS- and electrical characterization}

Secondary Ion Mass Spectroscopy (SIMS) provides a detailed chemical analysis of materials deposited on a planar substrate. For that deposition has been performed using the same parameters as for the TAP samples. As substrate a Silicon wafer is used, in order to avoid charging of the sample by the primary ion beam. A short description of the SIMS technique is given in section 3.5.2.

Spin-valve stacks like on the tip-like samples are additionally deposited on a substrate of silicon oxide. A $40 \mathrm{~nm}$ thick Cu layer is deposited beneath and on top of the stack for contacting the spin-valve system. The purpose of this sample is a direct measurement of the electric properties including the TMR. Three different types of samples are prepared, differing in barrier thickness $(1.0 \mathrm{~nm}, 1.5 \mathrm{~nm}, 2.0 \mathrm{~nm})$. These measurements have been obtained at the Department for Experimental Physics at the University of Bielefeld. 


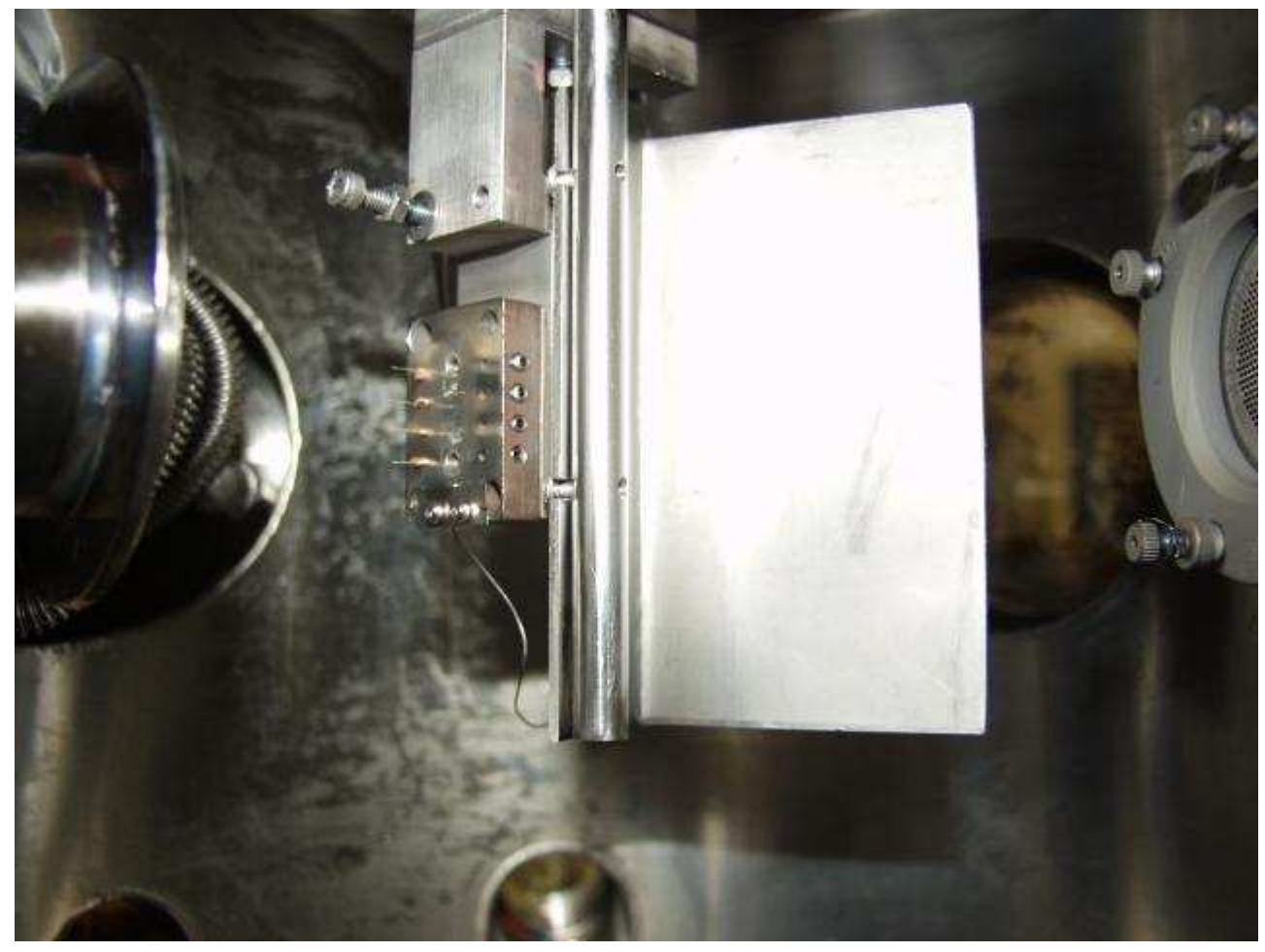

Figure 3.4: The specimen stage inside the ion beam sputter deposition chamber. The samples mounted on the heater are visible in the middle. The sheet nearby is the rotatable shutter that isolates the sample from the cloud of sputtered atoms, if necessary. The ion-source (right) and one of the targets (left) are identifiable. 


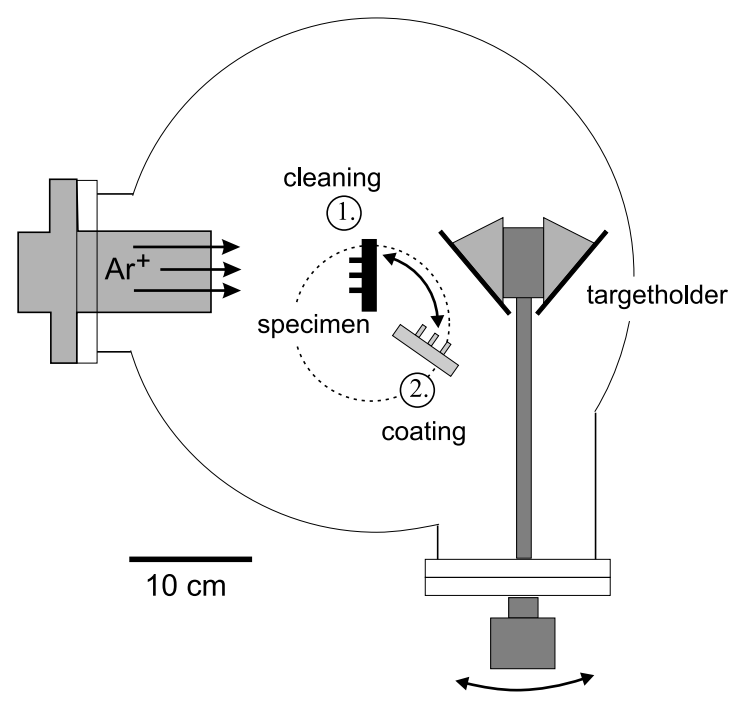

Figure 3.5: Geometry of the sputter deposition device (Schmitz, 2001). Switching between tip cleaning and coating is achieved by simple rotation of the specimen stage.

\subsection{Ion beam sputter deposition}

The layers to be investigated are deposited on prepared substrates using ion beam sputter deposition. The principle of ion beam sputtering is the removal of material from certain areas of the targets. These removed (sputtered) atoms are deposited on the surrounding materials including the tips situated in front of the target. The whole process takes place at room temperature and under vacuum conditions with a background pressure roughly $8 \cdot 10^{-8}$ mbar. A Kaufmann-source emitting Argon ions with energies of $500-600 \mathrm{eV}$ is used to remove atoms from the targets. The ion source, the targets and the specimens are water cooled.

As seen in Fig. 3.5, there are two different positions for the specimens. Position (1.) is required for tip-cleaning: without the cleaning of the tips right before deposition, the deposited material is not stable enough to withstand the conditions during the TAP measurement (Schleiwies and Schmitz, 2002). The cleaning process results in a slight roughening of the tip's surface.

Position (2.) represents the position for deposition. The Argon beam removes atoms from the target. The cloud of atoms expands uniformly in front of the target and is deposited on the specimen's surface. The specimen itself can be isolated with a shutter. This is important at the beginning of the deposition process, where contamination or oxides, adsorbed on the target surface must be removed ${ }^{1}$.

The complete sputtering process takes place in three steps. The first step is the cleansing of the targets and specimen (position (1.). Finally the deposition process is initiated (position (2.).

Pure metal layers are sputtered directly as described before. The preparation of

\footnotetext{
${ }^{1}$ the target holder is rotatable from outside, so that at most four targets of different materials can be inserted
} 
EXPERIMENTAL METHODS

Table 3.1: Overview of the parameters used for sputter deposition including deposition times and layer thicknesses $\left(d_{\text {layer }}\right)$.

\begin{tabular}{lccccc} 
Process & $\begin{array}{c}\text { Beam Current } \\
{[\mathrm{mA}]}\end{array}$ & $\begin{array}{c}\text { Beam Voltage } \\
{[\mathrm{V}]}\end{array}$ & $\begin{array}{c}\text { Ar pressure } \\
{[\mathrm{mbar}]}\end{array}$ & $\begin{array}{c}\text { Time } \\
{[\mathrm{s}]}\end{array}$ & $\begin{array}{l}d_{\text {layer }} \\
{[\mathrm{nm}]}\end{array}$ \\
\hline cleaning of target & 600 & 25 & $10^{-4}$ & 120 & - \\
cleaning of substrate & 500 & 10 & $10^{-4}$ & 45 & - \\
Py depos. & 550 & 12 & $10^{-4}$ & 360 & 10.0 \\
$\mathrm{Al}$ depos. & 550 & 12 & $10^{-4}$ & 120 & 2.5 \\
$\mathrm{Al}_{2} \mathrm{O}_{3}$ depos. & 550 & 12 & $10^{-4}$ & 300 & 2.0 \\
$\mathrm{Co}$ depos. & 550 & 12 & $10^{-4}$ & 400 & 15.0 \\
\hline Annealing & & - & & & 600 \\
@150...500 & - & - & - & & - \\
\hline
\end{tabular}

oxide layers is a bit more complicated; there are two possibilities. The first one is based on a subsequent oxidation process: after a layer of pure $\mathrm{Al}$ is deposited, the ion beam is shut off and oxygen at a certain partial pressure is introduced into the chamber for a few minutes? The other possibility is a reactive in-situ deposition: during the deposition of $\mathrm{Al}, \mathrm{O}$ is introduced into the chamber and the $\mathrm{Al}$ is oxidized right away. A mass-spectrometer provides monitoring of the partial O-pressure during the deposition. Tab. 3.1 shows that the deposition time of pure Al differs from that of $\mathrm{Al}_{2} \mathrm{O}_{3}$; the reason is the presence $\mathrm{O}$. It is presumably the Kaufmann-source has a lower ion output level in an oxygen atmosphere. Therefore the deposition rate decreases and the deposition time has to be increased to achieve a sufficient layer thickness.

The ion beam sputter deposition setup used in this work offered furthermore the ability to perform annealing during and after the deposition. For the annealing experiments, the deposited specimen was annealed right after the deposition. The heater has the ability to heat the specimen up to $700^{\circ} \mathrm{C}$ within approximately $5 \mathrm{~min}$.

\subsection{Field Ion Microscopy and 3D atom-probe to- mography}

The principles of field ion microscopy (FIM) were developed in the fifties of the last century. Thus the method is a well-known and very well understood in general. It was Erwin Müller, a german physicist working and living in Berlin, who invented these principles in 1951, 15 years after Müller invented the principle of field emission microscopy (FEM), a method that is rather similar to FIM, but not as effective.

\footnotetext{
${ }^{2}$ here a $\mathrm{O}$ pressure of $1 \cdot 10^{-4} \mathrm{mbar}$ and a duration of $\sim 10 \mathrm{~min}$ is applied for the subsequent oxidation
} 


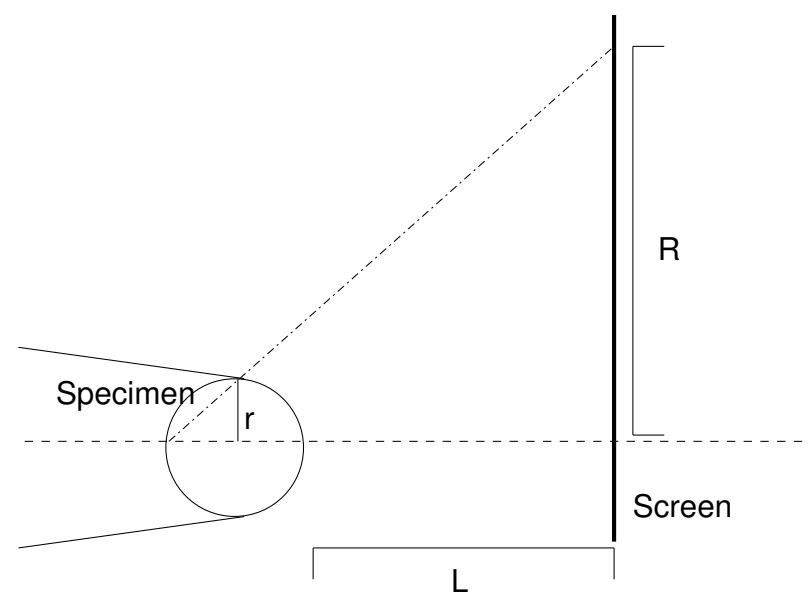

Figure 3.6: Schematic illustration of the magnification using the intercept theorems.

\subsubsection{Field ion microscopy (FIM)}

The basic principle of a field ion microscope is the application of an electric field between the sample and a phosphorescent screen, with a separating length $L=4$ $-20 \mathrm{~cm}$, where the sample is the anode. Directly in front of the screen Chevrontype micro-chanel-plates $(\mathrm{MCP})^{3}$ are adapted, in order to amplify the incoming events. The electric field is provided by a positive high base voltage $\left(U_{\text {base }}=3-\right.$ $15 \mathrm{kV}$ ) applied to the tip. The sample is a tiny tip with a curvature radius of 10 $-50 \mathrm{~nm}$ resulting in an enormous electric field of approximately $50 \mathrm{~V} / \mathrm{nm}$ upon the tips surface. The electric field can be derived using the relation

$$
E=\frac{U_{t i p}}{\beta \cdot r}
$$

where $\beta$ represents an image compression factor (normally $\sim 5-7$ ), which depends on the geometrical shape of the tip ${ }^{4}$ and $r$ is the curvature radius of the tip.

The magnification can be derived from (Fig. 3.6):

$$
\frac{R}{r}=\frac{L+2 r}{r}
$$

where $R$ is the radius of the impact point on the screen. Since $R>>r$, Eq. (3.2) can be rewritten as

$$
\frac{R}{r}=\frac{L}{r}
$$

Finally the magnification $M$ is given by

$$
M=\frac{L}{\kappa \cdot r}
$$

\footnotetext{
${ }^{3}$ secondary-ion-multiplier

${ }^{4}$ for an ideal spherical structure: $\beta=1$
} 
EXPERIMENTAL METHODS

where $\kappa$ is a compression factor $(\sim 0.7)$, which takes into account the influence of the tip's shaft.

The process takes place inside an evacuated chamber under UHV conditions. The pressure lies within the range of $10^{-9}$ mbar in order to prevent interaction of the evaporated atoms from the specimen with the rest gas. The temperature of the specimen lies in the range of $20 \mathrm{~K}$ to $50 \mathrm{~K}$ in order to reduce the thermal motion of the atoms and hence get a sharp image.

The image is not generated directly with evaporation of electrons from the specimen, but using an inert image gas like $\mathrm{He}$ or Ne. The imaging gas leaks into the chamber with a partial pressure of approximately $1 \cdot 10^{-5}$ mbar. When the tips positively charged by the application of a high voltage, the atoms or molecules of the image gas become polarized and are attracted to the tips surface (field induced adsorption).

In a free atom, the electrons are trapped in a potential with a symmetrical structure. To ionize an atom, it is necessary to expend ionization-energy. In the immediate area of the tips surface, the potentials of the imaging gas atoms are deformed asymmetrically. Therefore, the probability is higher that an electron tunnels through the potential barrier, but the electron can only tunnel to a free level above the Fermilevel of the tip material.

Field ionization directly upon the tip's surface is not possible: there must be a certain distance between the surface and the atoms (approximately $0.5 \mathrm{~nm}$ ). Therefore a first layer of He atoms is trapped on the tip's surface (ad-atoms). These atoms rest in this position even when a higher voltage is applied to the tip. The next atoms which lay on the ad-atoms reach the requested distance from the tip's surface and could be ionized when a sufficient field strength is reached. These ionized atoms are accelerated approximately along the electric field trajectories towards the MCP. The atoms impinging upon the MCP generate electron clouds, which illuminate a phosphorous screen. At protruding atoms on the tip's surface the electric field strength is increased relative to the remaining surface of the tip; these prominent areas are visible as brighter dots on the screen. To get an adequate image of the tip, it is necessary to apply a sufficient voltage to the tip. The value of this voltage depends on the tip's geometry, especially on the curvature radius and the shaft angle of the tip. Using the "best image voltage" $\left(U_{\text {best }}\right)$, the best possible image of the tip is achieved, i.e. one that represents a two dimensional projection of the three dimensional tip structure with optimum contrast. Fig. 3.7 shows an example of such a FIM-image representing the $\{011\}$-pole of a thinned $\mathrm{W}$ wire at $U_{\text {best }}$.

When the applied voltage is increased beyond $\left(U_{\text {best }}\right)$, the electrical field at the tip gets high enough to remove atoms at the tip's surface. The process is called fieldevaporation. The atoms are removed layer by layer. Due to the fact that the shaft angle of the sample is limited, the radius of curvature increases with increasing voltage. The magnification decreases simultaneously. Thus, the FIM principle is also usable for developing specimens to a specific geometry. The substrate tips used for this work have been processed up to $14 \mathrm{kV}$ in order to develop tips with a curvature radius of approximately $30-40 \mathrm{~nm}$.

The main difference between FIM and FEM is the fact, that in FIM the specimen is an anode and an imaging gas is necessary for imaging while in FEM, the specimen 


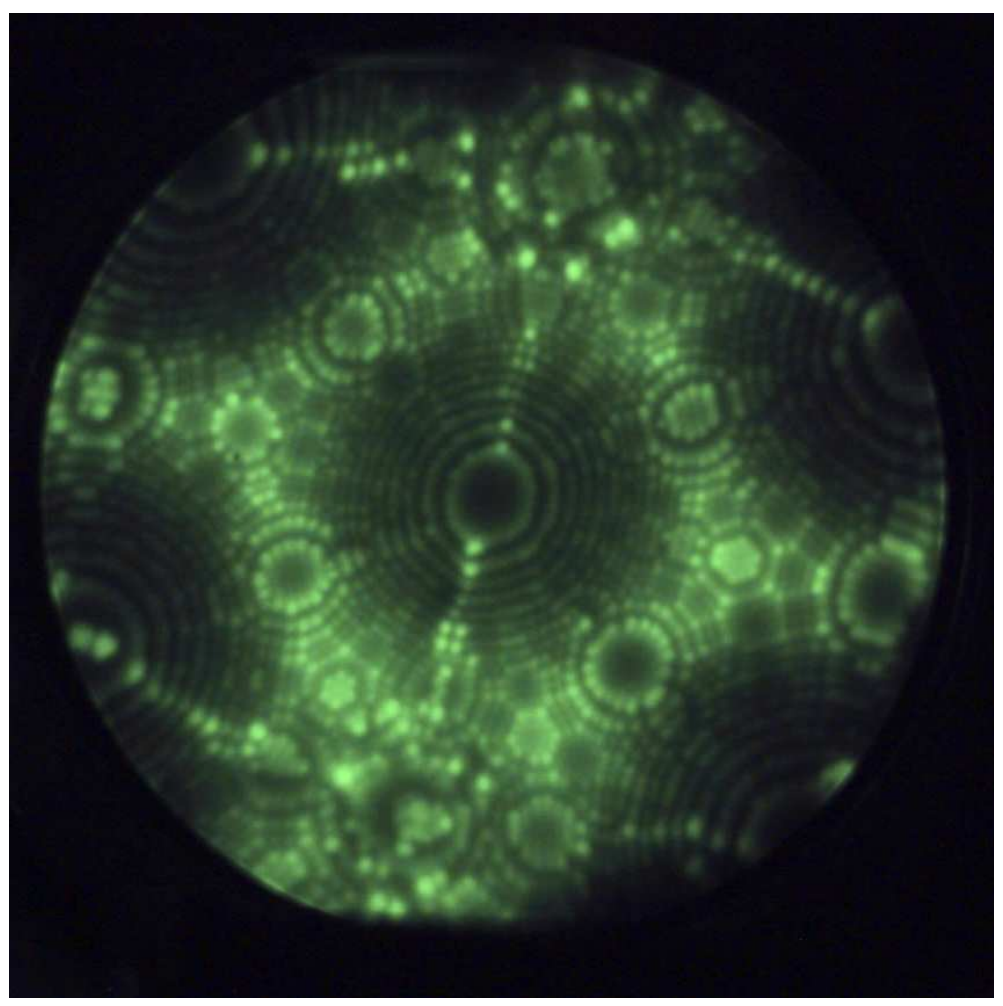

Figure 3.7: FIM image of the $\{011\}$-pole of $\mathrm{W}$ at $U=5.6 \mathrm{kV}$ and $T=20 \mathrm{~K}$.

is the cathode and the imaging is achieved by emitting electrons directly from the specimen. The main disadvantage of FEM compared to FIM is the lower resolution. Hence the electrons have a relatively high proper motion, only diffuse imaging can be achieved and therefore no atomic resolution is possible in FEM.

Further aspects about the theory of field ion microscopy can be found in Miller (1989).

\subsubsection{D atom-probe tomography (TAP)}

A tomographic atom probe (TAP) is based on the principle of field ion microscopy, therefore the technique is able to detect atoms with atomic resolution and chemical accuracy. The TAP is additionally equipped with a two-dimensional detection system developed at Rouen university, France (Blavette et al., 1993). The detector consists of 96 anodes mounted behind a set of MCP. Using high voltage pulses $U_{\text {pulse }}$ added to the base voltage $U_{\text {base }}$, atoms can be removed from the tip of the specimen. These atoms are accelerated along the electric field towards the MCP and the detector. The time of flight, which is measured between the pulse and the hit onto the detector, provides information about the mass of the atom by using the following relation based on the conservation of energy:

$$
E=\frac{m}{2} \cdot v^{2}=q \cdot U
$$




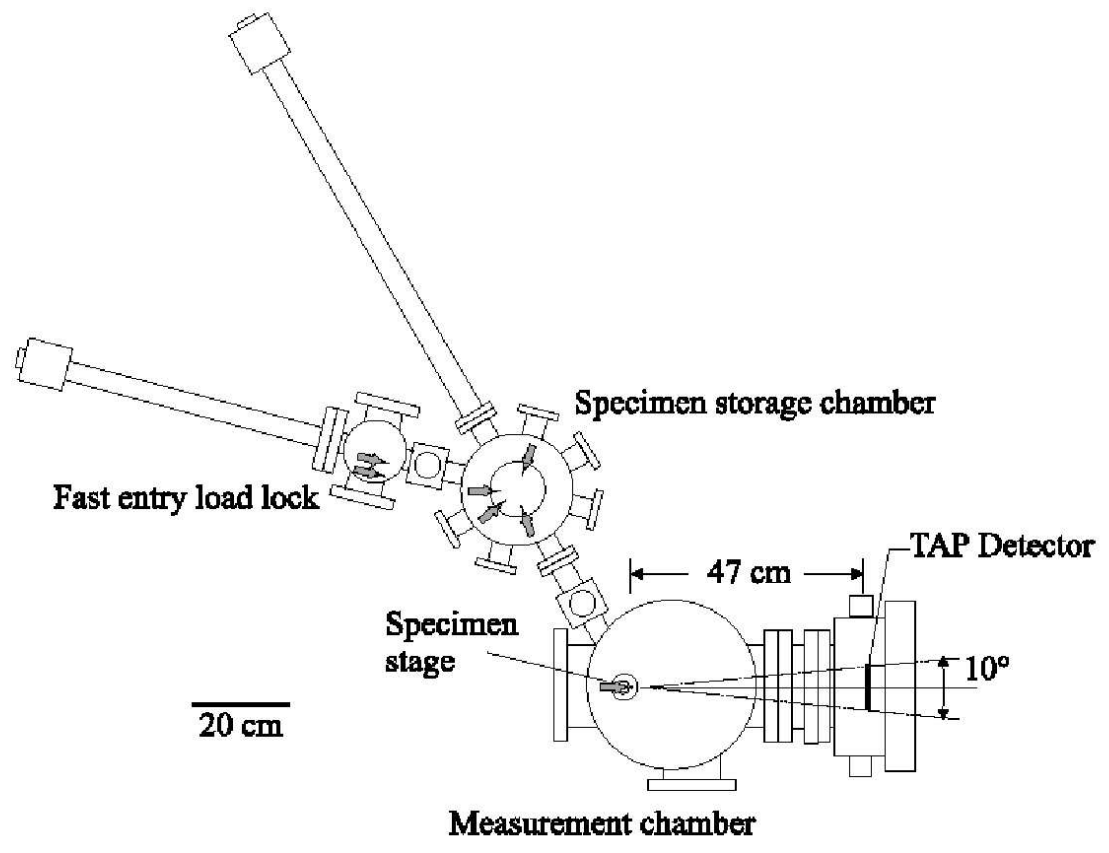

Figure 3.8: Sketch of the TAP setup in the Institute for Material Physics of the University of Göttingen.

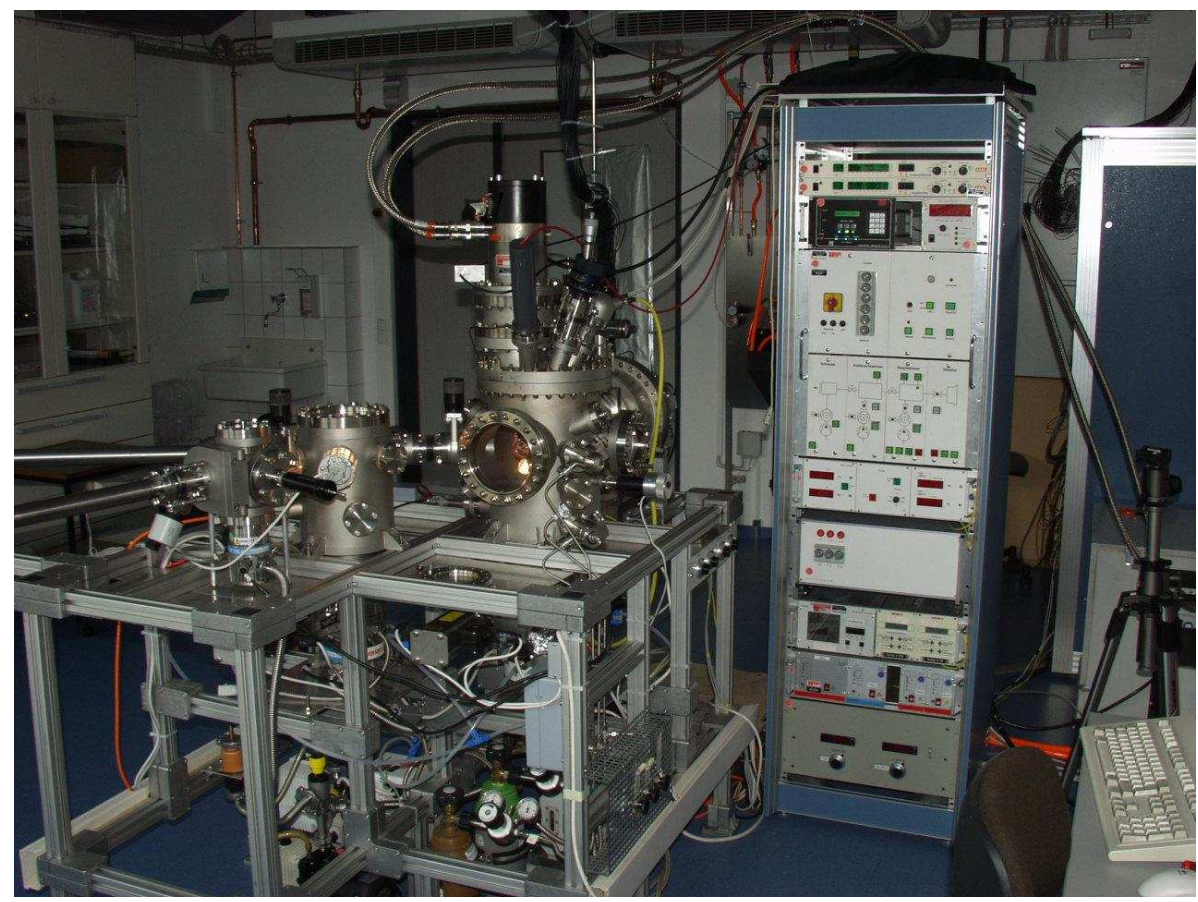

Figure 3.9: The TAP setup in the Institute for Material Physics of the University of Göttingen. 
where $t$ is the time of flight, $U$ the applied voltage to the tip (consisting of the sum of $U_{\text {base }}$ and $\left.U_{\text {pulse }}\right), L$ the flight length which is the length between the tip and the detector and $q$ the charge of each ion. Therefore (3.5) can be written as

$$
\frac{m}{q}=2 \cdot\left(U_{\text {base }}+U_{\text {pulse }}\right) \cdot\left(\frac{t}{L}\right)^{2} .
$$

By considering the impact position on the detector, the origin of each atom on the tips surface can be derived. This technique represents a major advantage compared with the conventional atom-probe technique introduced by Miller (1989). Using an MCP (see section 3.3.1), an electron cloud is generated on the detector's surface with each incoming ion and triggers at at least 3 to 5 anodes. From the distribution of the charge of the triggered anodes, the centered position $\left(x_{a}, y_{a}\right)$ of the electron cloud is derived. The origin of the ion is derived afterwards using the magnification:

$$
x=\frac{x_{a}}{M}, \quad y=\frac{y_{a}}{M} .
$$

The information concerning the depth in $z$-direction of the analysis is provided by deriving the number of evaporated atoms $N$ within a certain volume $V_{a t}$. For one atom, equations (3.1) and (3.4) yield

$$
\Delta z_{0}=\frac{M^{2}}{S_{D} \cdot A} \cdot V_{a t}=\frac{K}{U^{2}}
$$

where $V_{a t}$ the atomic volume, $S_{D}$ the detector efficiency, $A$ the detector's surface and

$$
\begin{aligned}
K & =\frac{L^{2}}{S_{D} \cdot Q} \cdot \frac{(E \beta)^{2} V_{a t}}{\kappa^{2}} \\
U & =U_{\text {base }}+U_{\text {pulse }} .
\end{aligned}
$$

A further correction is necessary because of the fact that the sample's surface does not have a planar structure but a spherical one. For example, if the sample's surface would have a planar structure, the consideration $z=N \cdot \Delta z_{0}=z_{0}$ would be adequate. The depth $z$ can now be refined by (Bas et al. 1995)

$$
z=z_{0}+z^{\prime}
$$

with

$$
z^{\prime}=R \cdot\left(1-\sqrt{1-\frac{\left(x^{2}+y^{2}\right)}{R^{2}}}\right)=R \cdot\left(1-\sqrt{1-\frac{\left(x_{a}^{2}+y_{a}^{2}\right)}{M^{2} R^{2}}}\right)
$$

Using the approximation $\frac{x^{2}+y^{2}}{R^{2} M^{2}}<<1, z^{\prime}$ can be written as

$$
z^{\prime}=\frac{\left(x_{a}^{2}+y_{a}^{2}\right)}{2 R M^{2}}
$$

Including all these corrections in the reconstruction algorithm, high atomic resolution in the $z$ direction is possible. The lateral resolution of the measurement 


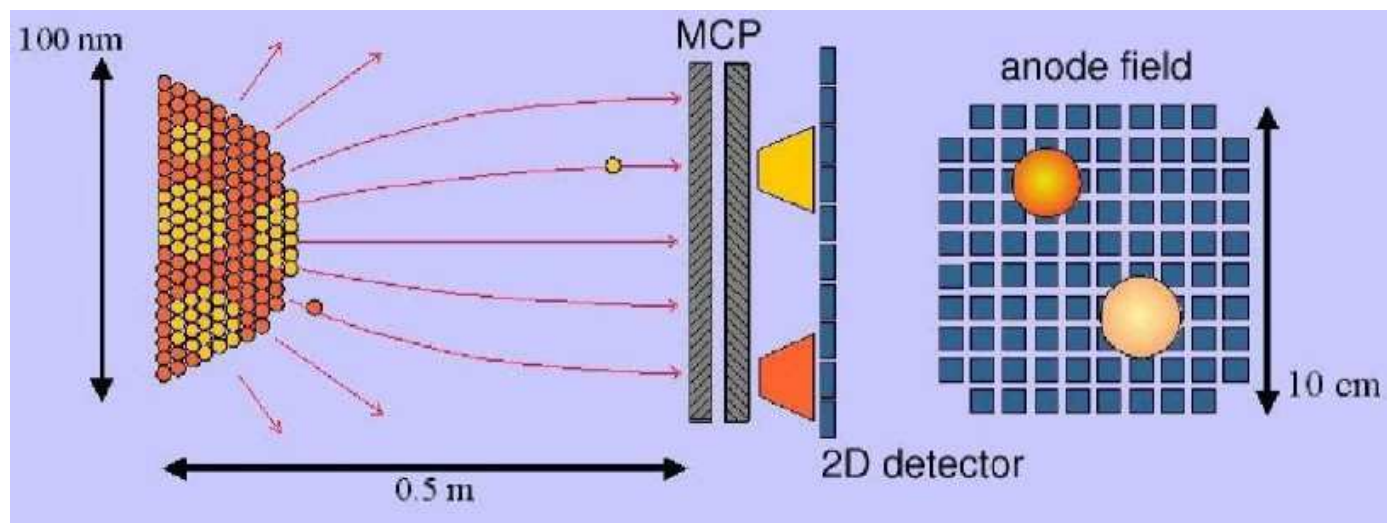

Figure 3.10: Schematic drawing showing the principles behind the tomographic atom-probe (TAP) (Schmitz, 2001).

technique is limited to $\sim 0.5 \mathrm{~nm}$ due to inaccuracies in the back-projection of the atom position on the sample's surface. A detailed description of the TAP installed at the Institute for Material Physics of the University of Göttingen can be found in Al-Kassab et al. (2003).

Measurements for the results in this work were performed under UHV-conditions $\left(\sim 5.0 \cdot 10^{-10} \mathrm{mbar}\right)$ at a temperature of $T \approx 75 \mathrm{~K}$. Atoms were removed by highvoltage pulses with a pulse frequency of $2000 \mathrm{~Hz}$. A pulse fraction of $22.5 \%$ pulse to base voltage, and a base voltage of $4-12 \mathrm{kV}$ delivered the best results. Approximately $80 \%$ of the prepared samples could be measured successfully.

\subsection{Data analysis}

The TAP measurement software provides basic geometry data which have to be analyzed in the next step. For this analysis, the software package AVS5 running on Unix workstations was used. AVS5 is an extensive visualization program which has been adapted for TAP analyses by specific routines, developed by the TAP working group of the University of Rouen/France and the group in Göttingen.

\subsubsection{Reconstruction procedure for layered structures}

The basic relation for the standard reconstruction procedure is given in equation (3.1). In this case, $\beta E$ is assumed to be constant during the whole measurement. This assumption is a good approximation for samples consisting of only one material and including small amounts of precipitates. In this work however, the measurement of thin layers is of interest, for which the field evaporation rate changes from layer to layer significantly (Jeske, 2001). Therefore, the standard routine is not useful and so an alternative procedure is applied for reconstruction, that assumes a constant shaft angle (Schmitz, 2001). The evolution of the curvature radius $R$ with increasing 


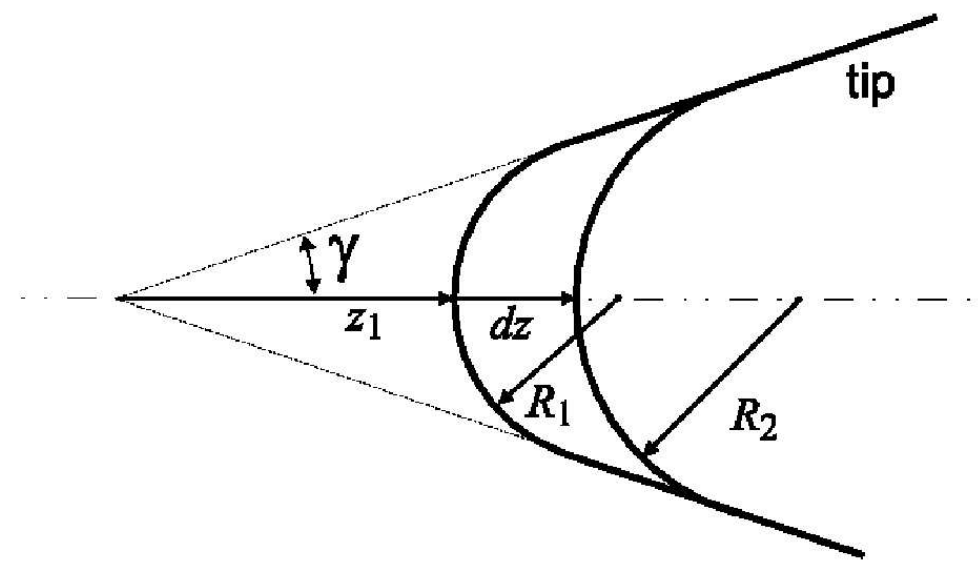

Figure 3.11: Geometrical structure of the reconstruction procedure based on initial $R$ and $\gamma$ (Schmitz, 2001).

depth $z$ can be derived using the relation

$$
\frac{d r}{d z}=\frac{\sin \gamma}{1-\sin \gamma}
$$

In order to derive $r(z)$ it is necessary to specify $\gamma$ and the initial value of $r$ and $\gamma$. These two parameters are adjusted, so that the calculated field evaporation rates correspond to the measured materials. Since radius and tip voltage are determined independently, the evaporation field strength can be calculated. Fig. 3.12 shows an example of a multilayer stack consisting of $\mathrm{Co}, \mathrm{Al}_{2} \mathrm{O}_{3}$ and $\mathrm{Py}$. It is obviously that the $\mathrm{Al}_{2} \mathrm{O}_{3}$ rich region has a different value than the surrounding elements.

\subsubsection{Concentration profiles}

To determine values for the concentration of atoms in a TAP measurement, an appropriate volume has to be specified in which the local composition is determined. This volume is moved through the measurement in specially defined steps. This way, a concentration profile can be derived on positions of interest inside the totally analyzed volume. The size of the evaluation volume and the step-size has to be chosen in accordance to the scientific problem. In order to get relevant statistics, every volume has to contain a minimum number of atoms to be analyzed and hence a minimum size, which depends on the concentration. The statistical error of concentration determination in time-of-flight mass spectrometry corresponds to the standard deviation $\sigma$ of a binomial distribution. Two concentration values can be interpreted as being different from each other, if the $2 \sigma$ intervals of each value do not overlap. The $2 \sigma$ boundary can be expressed in case of a binary system as a 


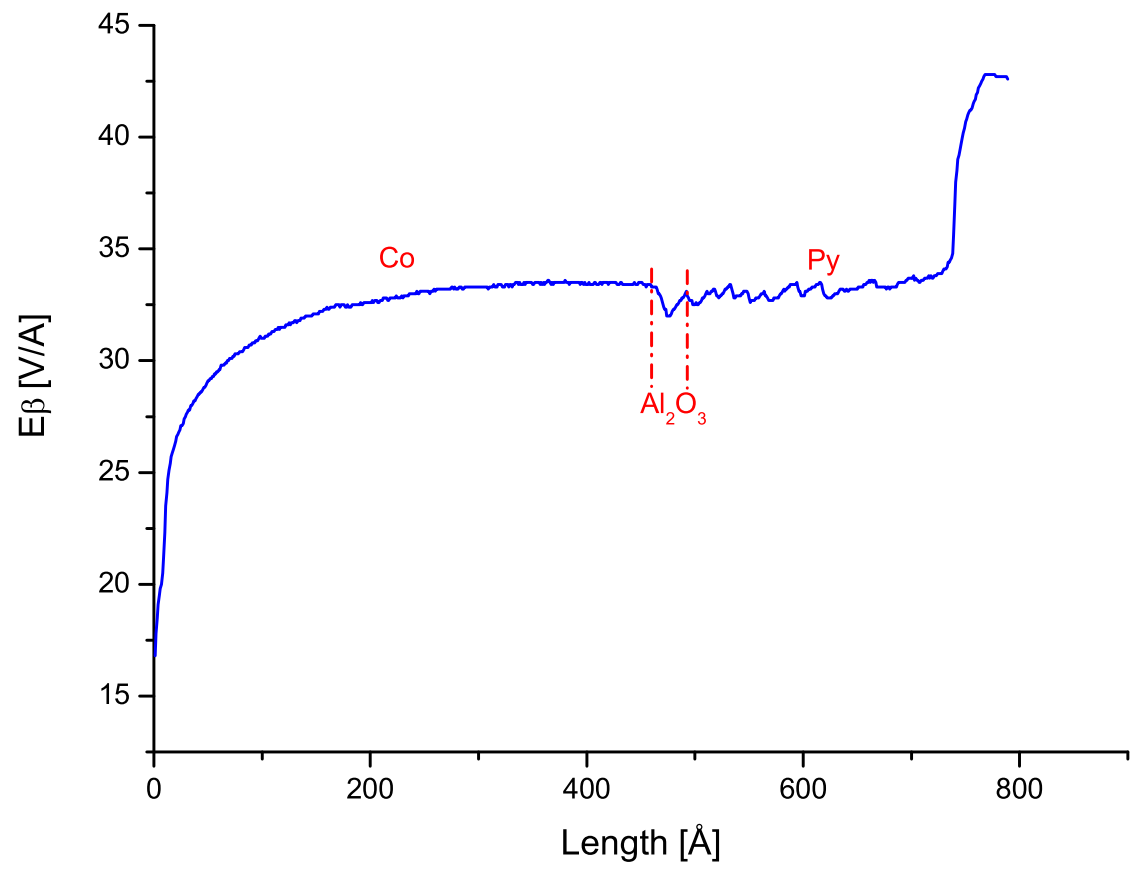

Figure 3.12: Field evaporation curve in a multilayer stack with a barrier consisting of Al-oxide. $E \beta$ varies with the properties of the material. The oscillations in the area of Py are an effect due to the adjustment of the field evaporation. 
function of the concentration $c$ and the number of atoms $N$ :

$$
2 \sigma=2 \cdot \sqrt{\frac{c \cdot(1-c)}{N-1}} .
$$

The evaluation volumes used in this work are cylindrical with a diameter of $3 \mathrm{~nm}$ and are aligned with their axis perpendicularly to the deposited layers. Local compositions are derived by a moving average along the cylinder axis. For that, the cylinder is subdivided into overlapping, disk-shaped boxes spaced by $1 \AA$ and a typical thickness of $3 \AA$.

\subsubsection{Derivation of the Gibbsian excess}

In order to classify interfacial segregation processes, it is necessary to investigate the quantity of segregated material along the segregation interface. The applied software can be used to derive the number of atoms within a certain volume using the same method as described in the previous section. A concentration profile is obtained by aligning a cylindrical evaluation volume perpendicular to the segregation plane. The result is a concentration profile in which the segregated material shows up as a peak. The integration of the corresponding peak after subtraction of the noise background yields the number of atoms $N_{a t}$ segregated to the interface. From the radius $r_{z}$ of the cylindrical evaluation volume, the surface can be derived from which the Gibbsian excess $\Gamma_{0}$ can then be calculated:

$$
\Gamma_{0}=\frac{N_{a t}}{\pi \cdot r_{z}}
$$

Eq. 3.13 has to be adapted to the measurement conditions, where the detector efficiency and the dimensions have to be considered. In addition the detector of the tomographic atom probe has a proven efficiency of 0.5 , i.e. is capable of detecting only half of the total number of atoms that hit the detector. Hence, the number of atoms has to be multiplied by a factor of 2 .

\subsection{Additional analysis methods}

\subsubsection{Transmission-electron-microscopy (TEM)}

The TEM used in this work is a Hitachi H-800 at the Institute for Material Physics at the University of Münster. The TEM is equipped with a La $\mathrm{B}_{6}$ cathode and operated with an acceleration voltage of $200 \mathrm{kV}$.

Although the structures considered in this work are near to the lower resolution limit of the instrument, the resolution is appropriate.

The device is used for magnification in order to measure the thickness of the layers. Images have been obtained with magnifications up to 150000 times. Additionally to planar samples, FIM tips were also investigated with TEM. 


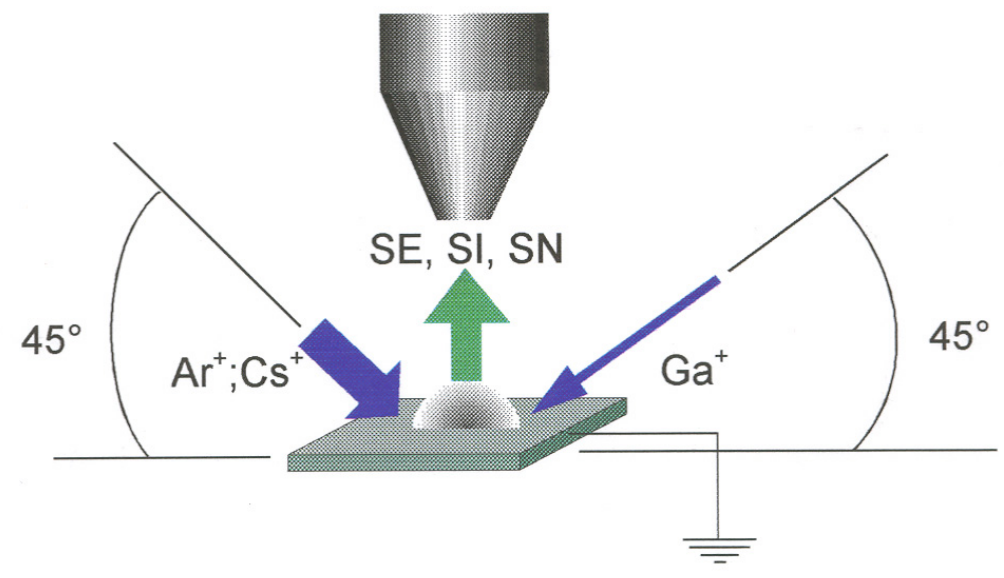

Figure 3.13: The principle of SIMS. The Ar beam erodes the sample while the Ga beam is responsible for sputtering of the ions which can be analyzed with TOF detector (Bankmann, 1998).

\subsubsection{Secondary-ion-mass-spectrometry (SIMS)}

SIMS uses a time-of-flight (TOF) analysis with a depth resolution of $2-5 \mathrm{~nm}$ and a lateral resolution of $\sim 1 \mu \mathrm{m}$. The deposited material is sputtered away by an $\mathrm{Ga}$ ion beam. In order to investigate deeper areas of the sample, an additional Ar ion beam is applied. In comparison to the Ar beam, the Ga beam can be focused to a tiny spot. Hence the analysis area can be well defined. This is not possible with the Ar source due to the fact that Ar is not liquid.

The ions of both beams hit the sample's surface with an angle of $45^{\circ}$. The Ga beam is applied only within a short timescale $(\sim 100 \mathrm{~ns})$. The removed ions create a cloud above the surface of the sample with a diameter in the range of $\mu \mathrm{m}$. Right after the Ga shot an extraction field is applied between the sample and the TOF detector. The removed ions are accelerated towards the detector, where the mass is derived by taking into account the time of flight of the ions. In order to analyze deeper areas of the sample, the Ar beam is applied to remove certain layers. During that time, the extraction field is aligned in the opposite way to prevent the sputtered ions from being accelerated towards the detector. The Ar beam has only a function of a "drilling system". After the application of the Ar beam the procedure is repeated. Fig. 3.14 shows the process sequence of iteration step during a SIMS measurements (the "Buncher" is an additional acceleration device, not in use during these measurements). The extraction bias represents the opposite field between the sample and the detector during the drilling by the Ar ions.

One decisive disadvantage of SIMS analysis is that it is not possible to give exact values concerning concentration and profile depth. Particularly, if samples consist of several different elements like it is the case in this work, the involved elements can influence the ionization probability of the secondary ions. These effects are called "matrix-effects". 


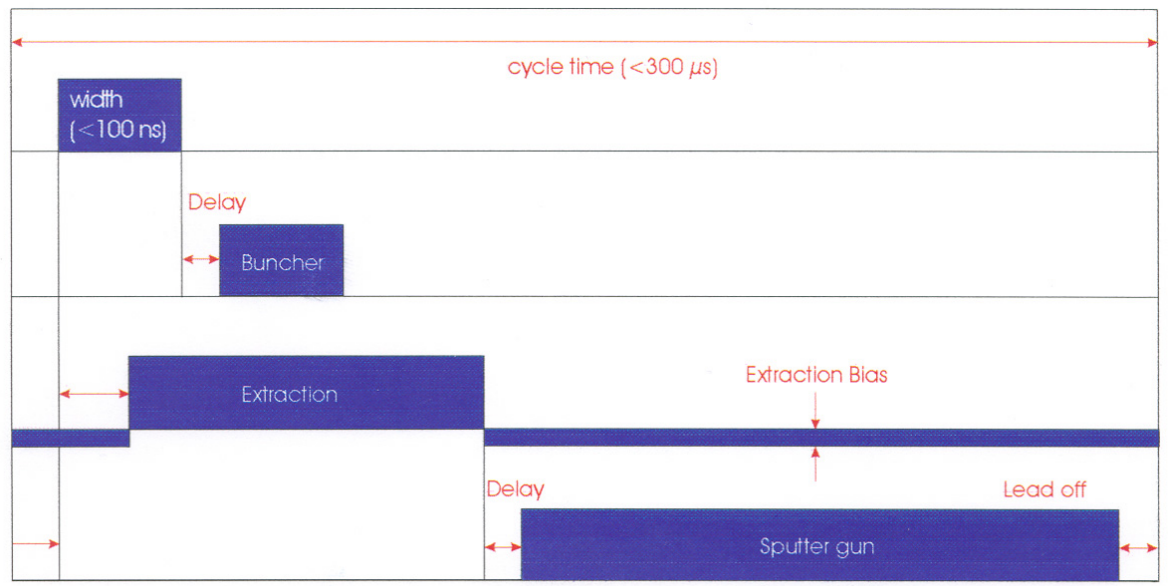

Figure 3.14: The principle of SIMS in chronological order. This diagram shows the order of process steps of one iteration during the SIMS measurement (Ion-TOF, 1996).

Table 3.2: Overview of the parameters used for SIMS measurement.

\begin{tabular}{lcccl}
\hline Ion-Source & $\begin{array}{c}\text { Beam Current } \\
{[\mathrm{A}]}\end{array}$ & $\begin{array}{c}\text { Beam Energy } \\
{[\mathrm{keV}]}\end{array}$ & $\begin{array}{c}\text { Primary Dose } \\
{\left[\text { Ions } / \mathrm{cm}^{2}\right]}\end{array}$ & Purpose \\
\hline $\mathrm{Ga}$ & $0.07 \cdot 10^{-12}$ & 25 & $1.43 \cdot 10^{13}$ & Analysis \\
$\mathrm{Ar}$ & $9.50 \cdot 10^{-9}$ & 1 & $1.43 \cdot 10^{17}$ & Sputtering \\
\hline
\end{tabular}



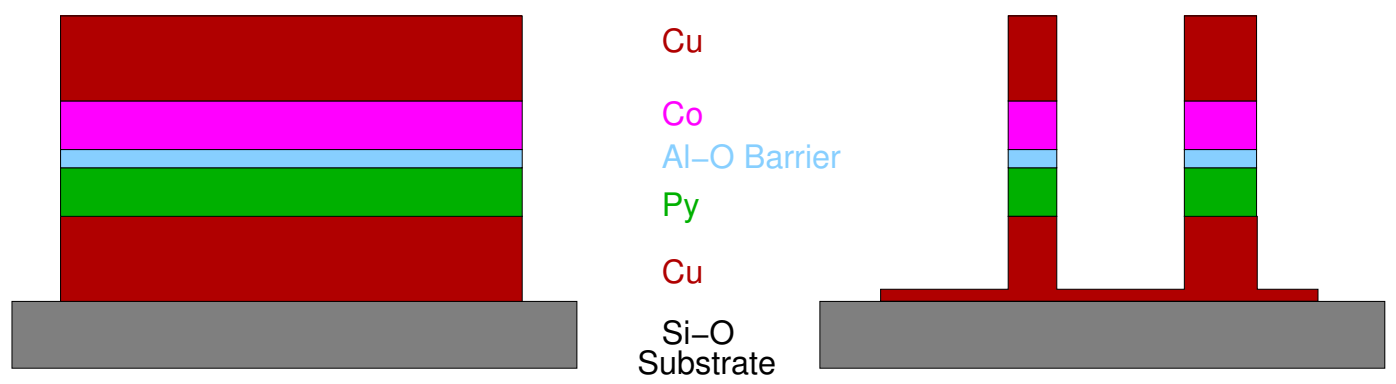

Figure 3.15: By etching a planar substrate prepared by ion beam sputter deposition (left, see section 3.2) several columns are prepared which are connected to the measurement stage by Au-electrodes at the top and at the bottom of the columns onto the $\mathrm{Cu}$ layers.

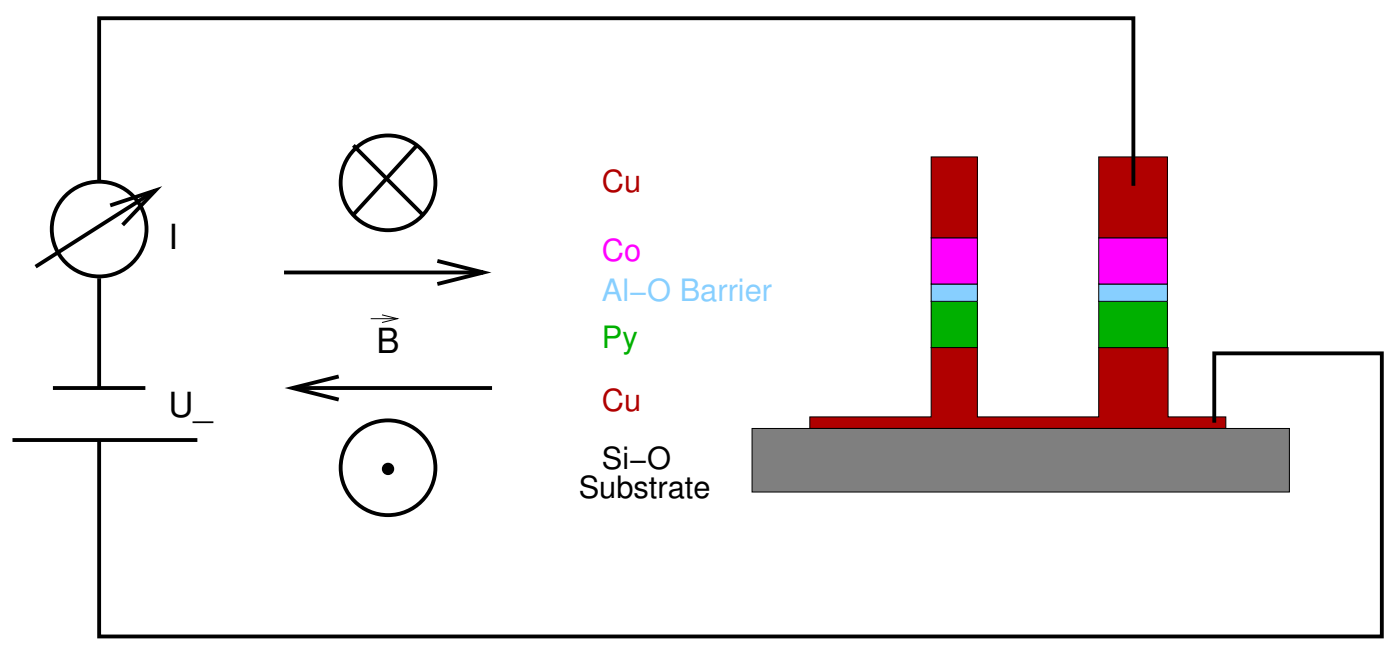

Figure 3.16: Principle of the measurement for obtaining data concerning the $U / I$ characteristic curves and TMR of the prepared spin-valve stacks. Direction and intensity of the magnetic field can be varied as required.

\subsubsection{Measurement of electrical properties and TMR}

The measurement of electrical properties like $U / I$ characteristic curves and TMR was obtained at the Physics Department of the University of Bielefeld. The samples were prepared as described in section 3.1.2. Several columns with a top surface of $200 \times 200 \mu \mathrm{m}$ were etched out of the planar sample (see Fig. 3.15) and afterwards contacted at the top and at the bottom with a simple Au-electrode (see Fig. 3.16). The measurements are controlled via a computer-program. The data obtained are fitted directly to the model by Brinkman et al. (1970) (see section 2.2) in order to characterize the barrier properties. 


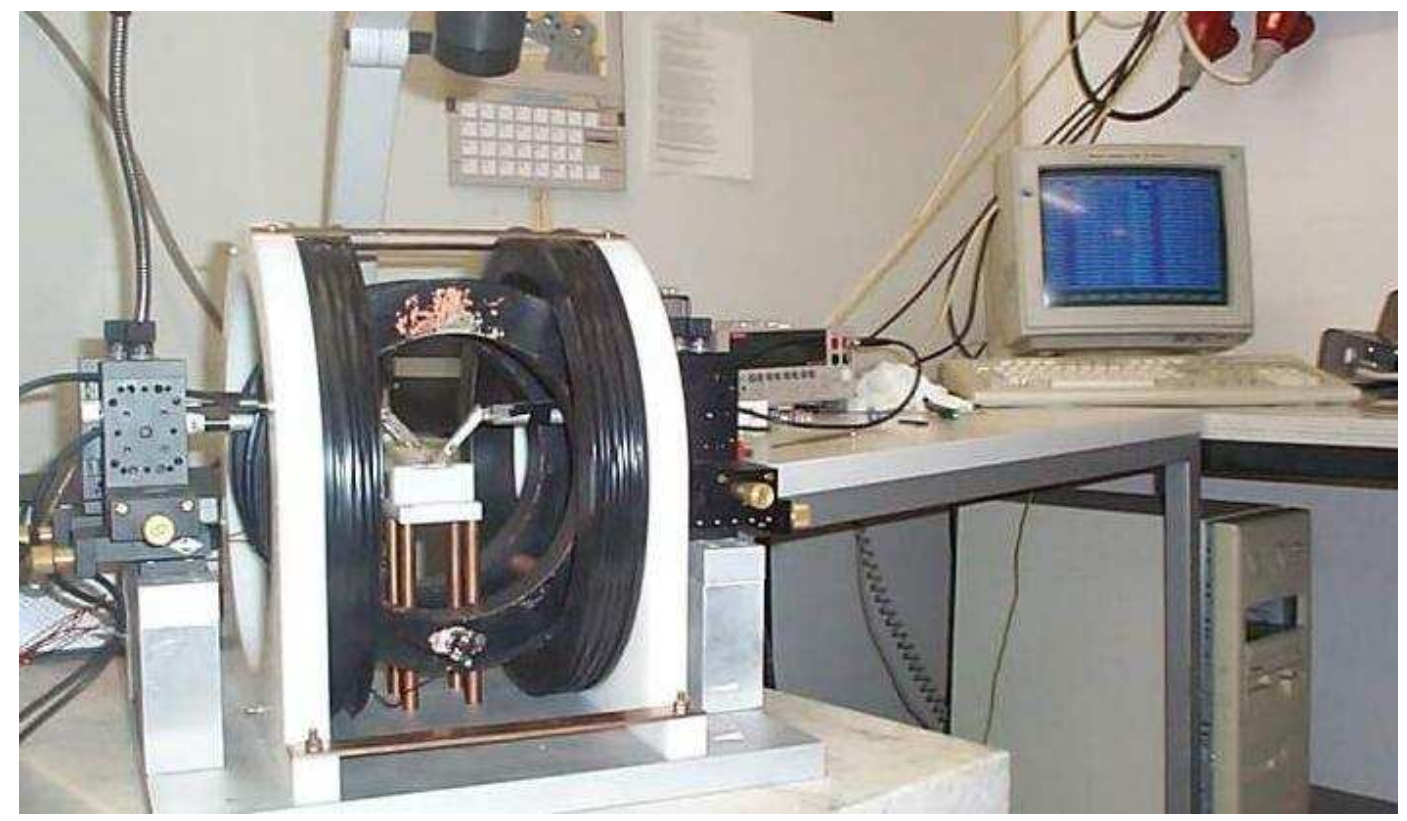

Figure 3.17: TMR measuring stage. The sample is positioned in the middle within the coils with which the magnetic field is generated (image taken from the web-site of Bielefeld University). 


\section{Chapter 4}

\section{Results}

\subsection{TAP results}

The first most important step in this work was to prepare samples containing oxide barriers for which a measurement with the TAP is possible. After optimizing the deposition process, successful measurements of the as-prepared state and later measurements of the annealed state were obtained that reveal changes in the system induced by the thermal treatment of the samples. The experiments done in this work have shown for the first time that TAP can be applied to non-conductive materials in a controlled manner.

\subsubsection{As-prepared state}

A typical reconstruction of the atom distribution inside the tri-layer is shown in Fig. 4.1, as obtained by the TAP measurement. The corresponding mass-spectrum is presented in Fig. 4.2. The mass spectrum reveals the expected peaks for single and doubly charged $\mathrm{Al}$ at $\sim 27 \mathrm{amu}$ and $\sim 13.5 \mathrm{amu}$, the peaks of doubly charged $\mathrm{Fe}$ at $\sim 27 \mathrm{amu}$, Co at $\sim 30 \mathrm{amu}$ and the various peaks of the Ni-isotopes surrounding those of Co. Since the high mass side of the Co peak overlaps with two of the $\mathrm{Ni}$ peaks in a range of $\sim 0.9 \mathrm{amu}$, it is not possible to identify single events of these atoms with $100 \%$ confidence. However, reliable composition data are obtained by correcting concentration profiles for this peak overlap. The correction is done by measuring the ${ }^{58} \mathrm{Ni}^{2+}$ isotope separately from the ${ }^{59} \mathrm{Co}^{2+}$ and the other isotopes and calculating the concentration values using (see Fig. 4.3):

$$
\begin{aligned}
& C_{(N i)}=I_{N i}^{[[I]}-I_{C o}^{0} \cdot \frac{\eta_{1}}{\eta_{2}}+I_{N i}^{[[I]} \cdot \frac{\eta_{3}+\eta_{4}}{\eta_{4}} \\
& C_{(C o)}=I_{C o}^{0} \cdot \frac{\eta_{1}+\eta_{2}}{\eta_{2}}-I_{N i}^{[[I]} \cdot \frac{\eta_{3}}{\eta_{4}}
\end{aligned}
$$

Similarly, the peak at $27 \mathrm{amu}$ must be evaluated carefully, since it is a result of both 


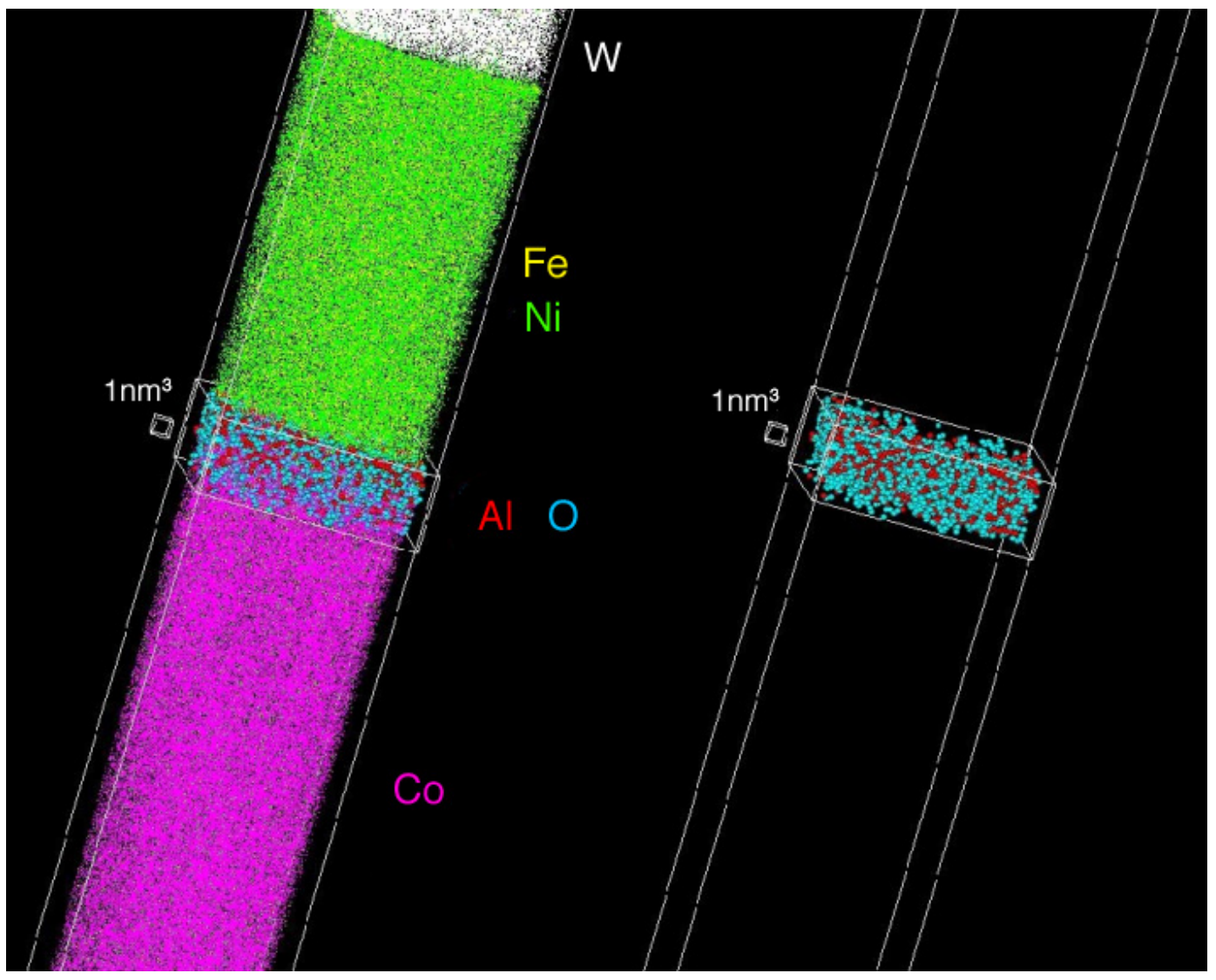

Figure 4.1: TAP reconstruction of a layered system with an oxide barrier. Each dot represents an atom of its kind. The atoms of $\mathrm{Al}$ and $\mathrm{O}$ are enlarged for better illustration. In the right figure only the $\mathrm{Al}$ and $\mathrm{O}$ atoms are visible. The lightgrey dots on top of the left figure represent $\mathrm{W}$ atoms, belonging to the top of the substrate. 

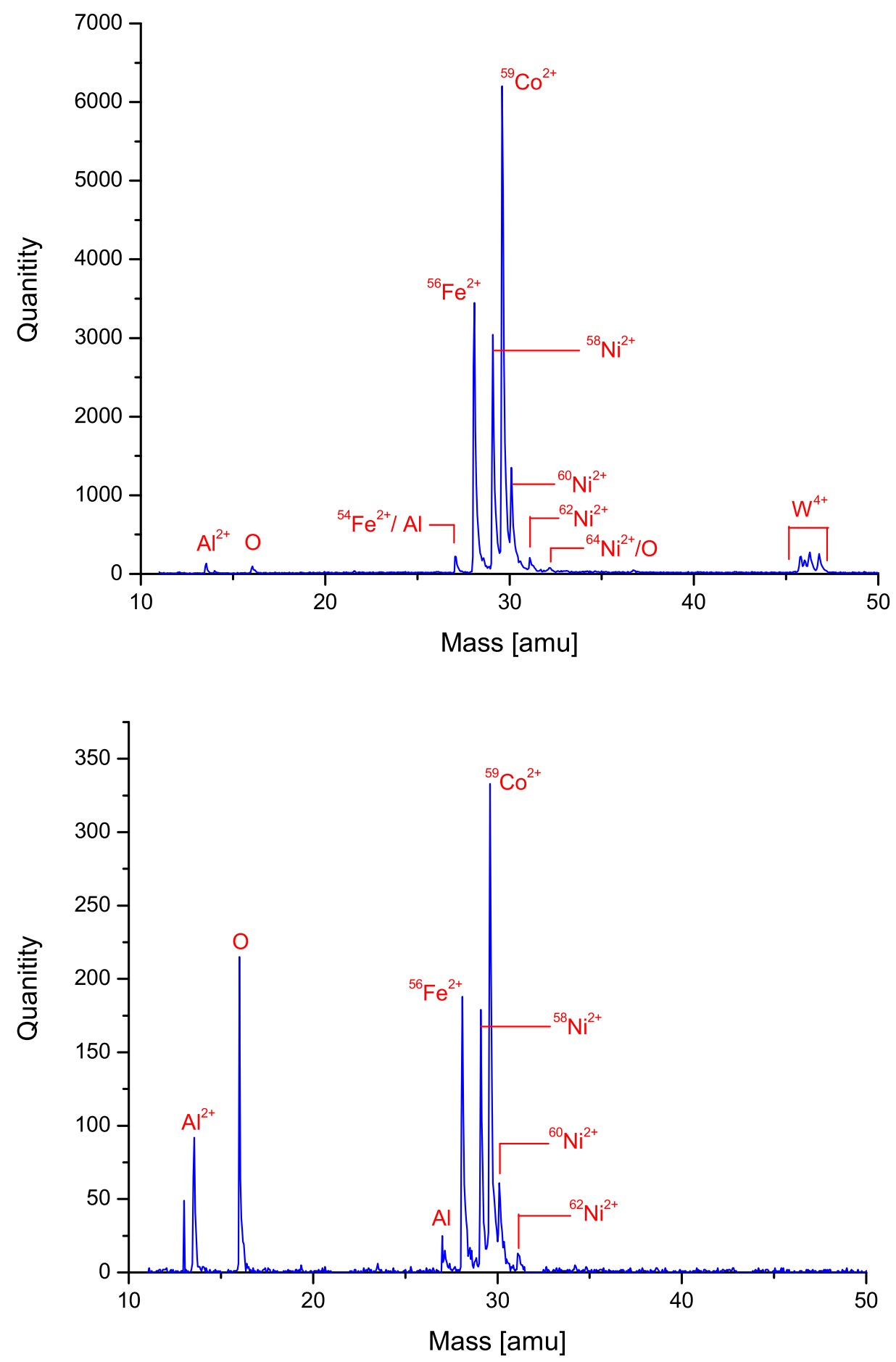

Figure 4.2: Mass spectrum of the TAP measurement shown in Fig. 4.1. Original spectrum of the total measurement (up) and corrected spectrum of the barrier region (bottom). Blank regions at about $22 \mathrm{amu}$ and $32 \mathrm{amu}$ are a result of the mass correction (see text). 


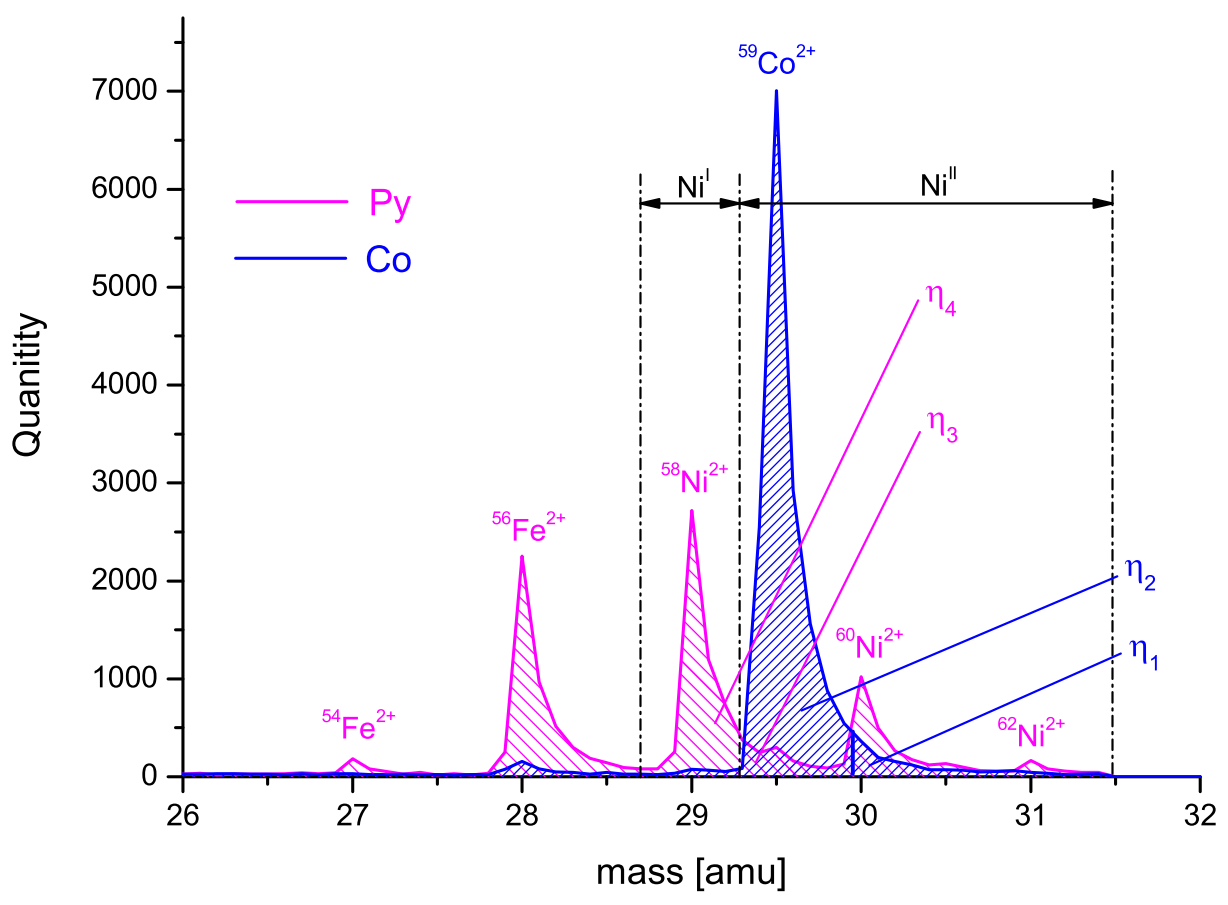

Figure 4.3: Correction areas and variables to correct the mass overlap of Co and the involved $\mathrm{Ni}$ isotopes.

$\mathrm{Al}^{+}$and ${ }^{54} \mathrm{Fe}^{2+}$ events. Taking into account the abundance of the Fe isotopes, the fraction of $\mathrm{Al}^{+}$and $\mathrm{Fe}^{2+}$ can be derived from

$$
\begin{aligned}
C_{\left(A l^{+}\right)} & =I_{\left(A l^{+}\right)}-\frac{0,06}{0,94} \cdot I_{\left(56 F e^{2+}\right)} \\
C_{\left(F e^{2+}\right)} & =\left(1+\frac{0.06}{0.94}\right) \cdot I_{\left(56 F e^{2+}\right)} .
\end{aligned}
$$

$\mathrm{Al}$ and $\mathrm{O}$ atoms are not always evaporated as individual ions, as indicated by events with a mass of $\sim 21.5 \mathrm{amu}$ or $\sim 43 \mathrm{amu}$. These events represent Al-O ions that are one- and two-fold ionized, respectively. Inside the barrier, a peak at $\sim 32 \mathrm{amu}$ indicates the appearance of molecular oxygen. To provide a useful analysis and reconstruction, these molecular events are split into two events of the respective atoms and assigned to the related mass peaks of the individual species. In the case of the peak at $\sim 32 \mathrm{amu}$, this correction was only done inside the barrier defined by a significant amount of oxygen. Outside the barrier, the peak is dominated by the $\mathrm{Ni}$-isotope ${ }^{64} \mathrm{Ni}^{2+}$ so that the events are assigned to the local $\mathrm{Ni}$ fraction. This reasonable correction procedure produces "blind" zones in the corrected mass spectrum, where the molecular events have been deleted and redistributed to other ranges of the mass spectrum (see second plot in Fig. 4.2).

Fig. 4.4 presents concentration profiles determined through a barrier, produced by in-situ oxidation at a partial pressure of oxygen of $1 \cdot 10^{-4}$ mbar. According to the width at half maximum of the oxygen profile, the barrier has a thickness of $\sim 2 \mathrm{~nm}$. 


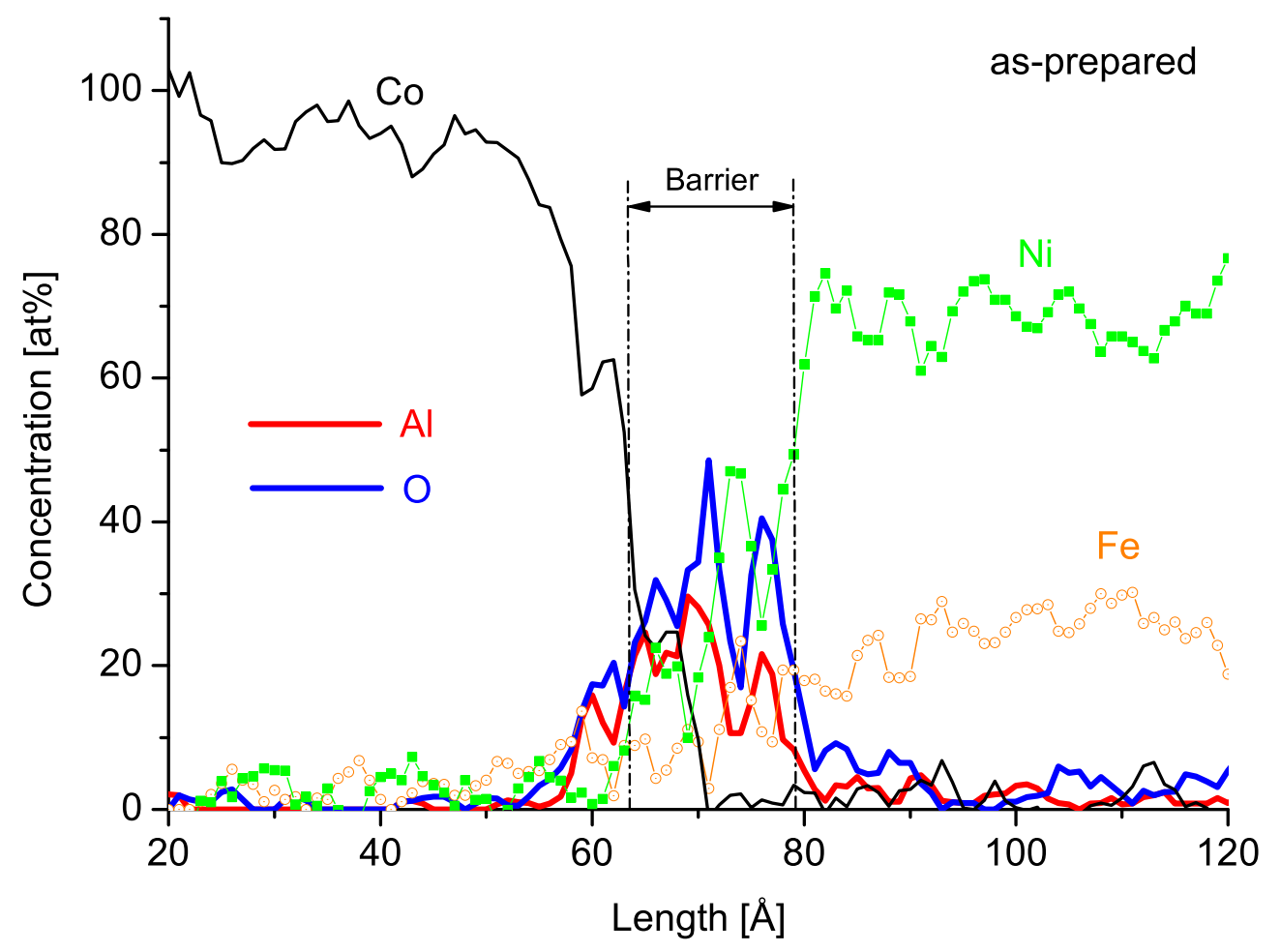

Figure 4.4: Concentration profiles across a barrier formed by Al deposition under a partial oxygen pressure of $1 \cdot 10^{-4}$ mbar (moving average, box length $0.7 \mathrm{~nm}$, i.e. about 250 atoms evaluated per data point). 


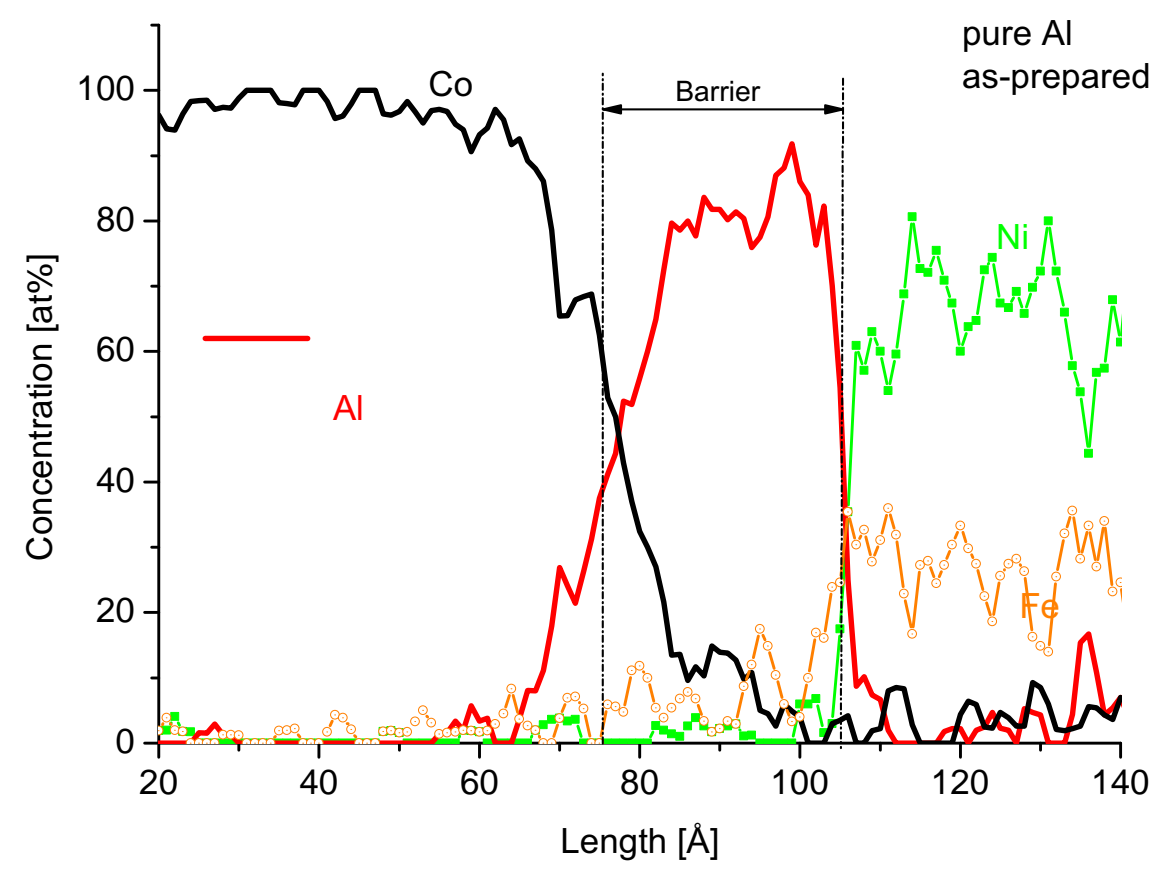

Figure 4.5: Concentration profile of a measurement through a pure Al "barrier" (moving average, box length $0.5 \mathrm{~nm}$, i.e. about 200 Atoms evaluated per data point).

Integration of the $\mathrm{O}$ and the $\mathrm{Al}$ content inside this region yields 1.65 times more $\mathrm{O}$ than $\mathrm{Al}$, so that the barrier composition closely matches the stoichiometry of $\mathrm{Al}_{2} \mathrm{O}_{3}$ under these conditions.

The significant contribution of $\mathrm{Ni}$ and $\mathrm{Fe}$ to the barrier is surprising: when $\mathrm{Al}$ is sputtered without any $\mathrm{O}$, the intermixed area between Py and the "barrier" is very sharp $(\sim 0,5 \mathrm{~nm})$ and no $\mathrm{Ni}$ or $\mathrm{Fe}$ is found inside the Al layer as shown in Fig. 4.5. The significant asymmetry between the two interfaces of the Al layer reflects the order of deposition. Depositing Al on top of Py causes less intermixing than depositing $\mathrm{Co}$ on the already grown Al layer. Prepared samples with the inverted layer sequence $\left(\mathrm{Co} / \mathrm{Al}_{2} \mathrm{O}_{3} / \mathrm{Py}\right.$ ) show a similar amount of $\mathrm{Ni}$ and $\mathrm{Fe}$ inside the barrier, so that the increased level of $\mathrm{Ni}$ and $\mathrm{Fe}$ in presence of oxygen cannot be explained by an artefact of field evaporation or by ion beam mixing during layer deposition. Some chemical reaction inside the setup caused by the presence of $\mathrm{O}$ must occur which makes $\mathrm{Ni}$ and $\mathrm{Fe}$ move from the Py layer into the oxide barrier.

Additional measurements were performed with specimens produced with different partial pressures of oxygen during the in-situ deposition and with a-posteriori oxidized specimens. Tab. 4.1 summarizes the investigated structures and the obtained barrier compositions.

Comparing the results of different deposition conditions, it is obvious that the oxy- 
Table 4.1: Measured oxygen/aluminum ratio inside the barrier.

\begin{tabular}{lclc} 
preparation method & $\begin{array}{c}\text { Oxygen pressure } \\
{[\mathrm{mbar}]}\end{array}$ & \multicolumn{1}{c}{ O/ Al ratio } \\
& $1 \cdot 10^{-4}$ & $1.65,1.51,1.81,1.33,1.49,1.55$ & $\mathbf{1 . 5 6} \pm 0.16$ \\
in-situ oxidation & $6 \cdot 10^{-5}$ & $1.33 ; 1.55 ; 1.63$ & $\mathbf{1 . 5 0} \pm 0.16$ \\
in-situ oxidation & $3 \cdot 10^{-5}$ & 1.51 & $\mathbf{1 . 5 1} \pm 0.10$ \\
in-situ oxidation & $1 \cdot 10^{-5}$ & $0.93 ; 0.97 ; 1.13$ & $\mathbf{1 . 0 0} \pm 0.10$ \\
in-situ oxidation & $1 \cdot 10^{-4}$ & $0.57 ; 0.76$ & $\mathbf{0 . 6 7} \pm 0.10$ \\
a-posteriori oxidation & & &
\end{tabular}

gen content of the barrier decreases significantly with decreasing partial pressure of oxygen. Given a sufficient oxygen pressure, the measured composition closely matches the expected stoichiometry, which is noteworthy, since the atom probe is a calibration free technique. Obviously, a quantitative measurement by atom-probe tomography is possible even with the non-conductive oxide material.

An important observation is furthermore, that oxygen rests inside the barrier and does not diffuse into the related metals. Obviously, the in-situ oxidation with a maximum partial pressure of $10^{-4}$ mbar is useful for the preparation of oxide-barriers and doesn't affect the chemistry of the surrounding electrode materials: no overcharging with oxygen occurs under these conditions. On the other hand, the aposteriori natural oxidation at room temperature with a partial oxygen pressure up to $\sim 10^{-4}$ mbar after the deposition of $\mathrm{Al}$ is by far not sufficient to reach the equilibrium stoichiometry.

Another question which needs to be addressed is the lateral integrity of the oxide layer. The 3D-analysis of the TAP shows that the oxide barriers are laterally homogeneous on the length scale of the analyzed volume. This is demonstrated by two-dimensional composition maps generated from the atomic reconstructions as shown in Fig. 4.6. In the sequence of the maps spaced by $8 \AA$ a concentric ring structure is observed, which is the natural consequence of the curved layer geometry on top of the substrate tips. The systematic growth of the oxide ring and its rather dense structure confirms that the oxide layer is indeed compact without significant pinholes weakening the isolating barrier. Furthermore - and more important in this context - it indicates that preferential field evaporation and thus local magnification effects have no severe impact on the analysis. Indeed, if the evolution of the oxide ring with the analysis depth is compared with that of an aluminum ring in a purely metallic specimen (see Fig. 4.7), it is found that the evaporation proceeds very similar in both cases. Furthermore the ring radius $r$ agrees well with a spherical tip shape as indicated by the solid lines calculated according to

$$
r=\sqrt{R^{2}-(R-\triangle z)^{2}}
$$

where $R$ and $\triangle z$ denotes the curvature radius of the layer and the increment of analysis depth, respectively. Thus, the oxide layer in the reconstructed volume 


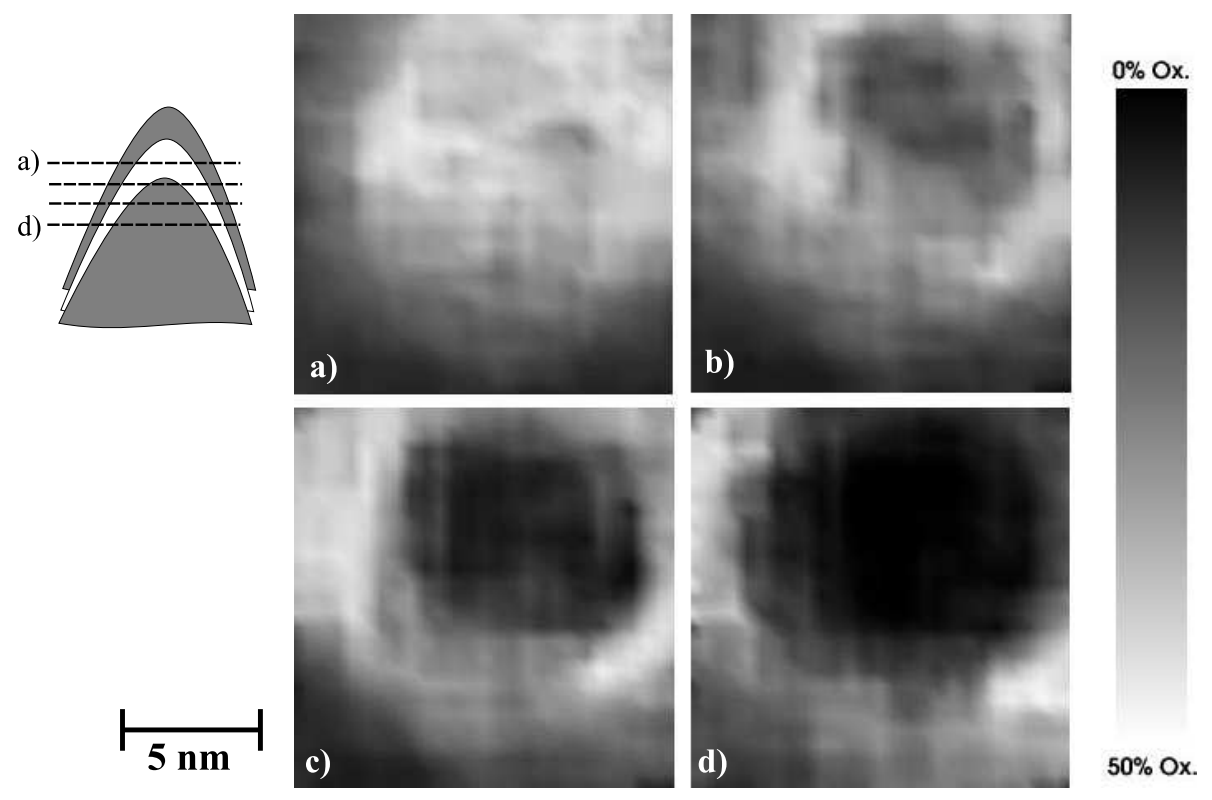

Figure 4.6: 2D-composition maps of the oxygen concentration of a sample prepared by in-situ oxidation with $\sim 10^{-4}$ mbar partial pressure of oxygen. Each of the four images represents a planar cut through the analyzed volume spaced by $0.8 \mathrm{~nm}$ to each other.

closely follows the shape of the Py-covered substrate tip, which makes clear that the evaporation sequence of the atoms is not disturbed by the pronounced difference in the theoretically expected evaporation fields.

\subsubsection{Annealed state}

Several important phenomena were observed in annealed state showing a clear dependence on the annealing temperature. The first effects showing up due to annealing have been observed at temperatures of about $200^{\circ} \mathrm{C}$. Samples annealed at $150^{\circ} \mathrm{C}$ show the same features as samples in the as-prepared state, suggesting that the first effects of thermal treatment occur within the range of $150^{\circ} \mathrm{C}$ and $200^{\circ} \mathrm{C}$.

One essential observation is a segregation of mostly Fe and additionally some Ni at the interface of the barrier towards the Co side and the other one is a significant reduction of $\mathrm{Ni}$ and $\mathrm{Fe}$ impurities inside the barrier. Both effects are demonstrated by the composition profiles in Fig. 4.8 determined after annealing at $200^{\circ} \mathrm{C}$ and $400^{\circ} \mathrm{C}$ for $10 \mathrm{~min}$ at a time in comparison to the as-prepared state (see Fig. 4.4). A natural explanation is that at least part of the $\mathrm{Fe}$ and $\mathrm{Ni}$ segregated to the Co interface were previously located within the barrier. Two-dimensional composition maps of the barrier region (Fig. 4.9) reveal that the segregating component $\mathrm{Fe}$ is (apart from statistical fluctuations) distributed homogeneously along the interface. The segregation amplitude increases significantly with the annealing temperature. This is demonstrated by a plot of the experimentally determined Gibbsian interfacial 


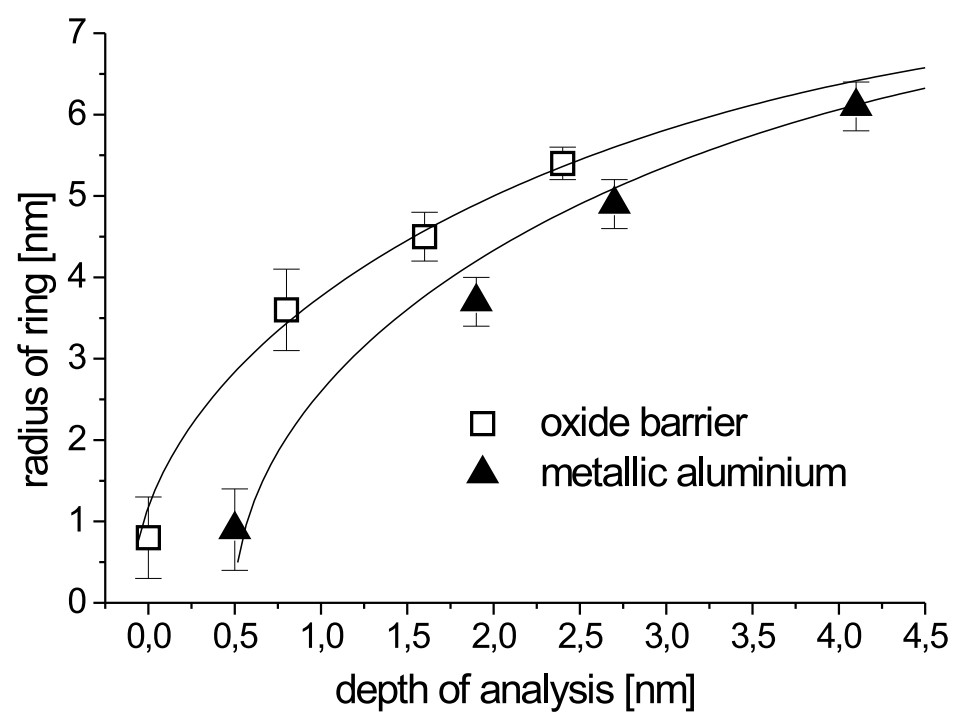

Figure 4.7: Evolution of the size of the oxide ring with advancing analysis depth in comparison to a specimen with purely metallic layers. Solid lines are calculated by Eq. 4.5

excess of iron versus the annealing temperature as presented in Fig. 4.10, The linear increase with temperature can not be explained by a physical model yet. It has to be seen as an experimental fact. The linear behavior of the excess as shown in Fig. 4.10 can be described by

$$
\Gamma_{0}(T)=(1.36 \cdot T-16.14) \cdot 10^{13} \mathrm{~cm}^{-2}
$$

and is only to be seen as a phenomenological description. Up to a temperature of $150^{\circ} \mathrm{C}$ no appreciable modification is seen. Thus, the onset of thermal reaction must occur in the temperature range between $150^{\circ} \mathrm{C}$ and $200^{\circ} \mathrm{C}$.

Beside the segregation at the barrier/Co interface, local breakthrough structures develop during heat treatments at temperatures of at least $300^{\circ} \mathrm{C}$. At the same time the segregation at the interface tends to become discontinuous. This is illustrated in Fig. 4.11, which represents an iso-concentration surface at 15 at $\%$ Fe. Its light face is directed towards decreasing Fe content. The upper nearly horizontal surface approximately marks the position of the barrier/Py interface. Vertical Fe enriched channels are seen, crossing the barrier towards the segregation zone at the bottom. Additionally to these local defects inside the barrier, grain boundary segregation of $\mathrm{Ni}$ to $\mathrm{Co}$ grain boundaries and $\mathrm{Co}$ to $\mathrm{Py}$ is observed in other samples, if grain boundaries are present in the analyzed volume (see Fig. 4.15).

In the temperature range beyond $400^{\circ} \mathrm{C}$ the structure of the samples gets more and more mixed up, while the atoms that form the barrier rest in their initial position. The atoms of the electrode layers move through the barrier layer and get completely mixed while the Fe segregation structure at the barrier/Co interface vanishes. This effect is first observed at annealing temperatures of $450^{\circ} \mathrm{C}$. At this temperature 

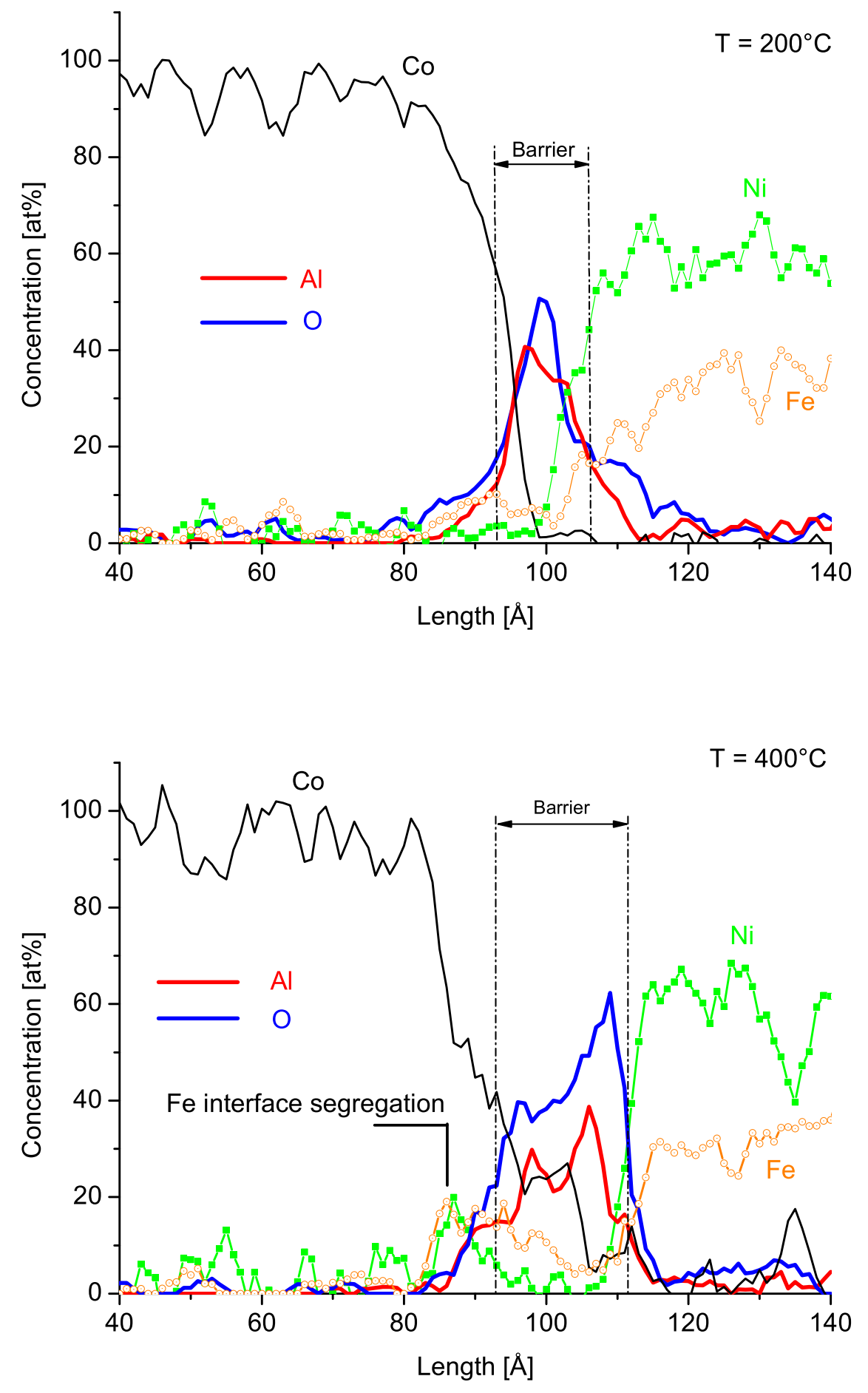

Figure 4.8: Concentration profiles of annealed samples at $200^{\circ} \mathrm{C}$ and $400^{\circ} \mathrm{C}$. Both samples show $\mathrm{Fe}$ and $\mathrm{Ni}$ segregation at the barrier/Co interface and reduced content of $\mathrm{Ni}$ and $\mathrm{Fe}$ within the barrier. 

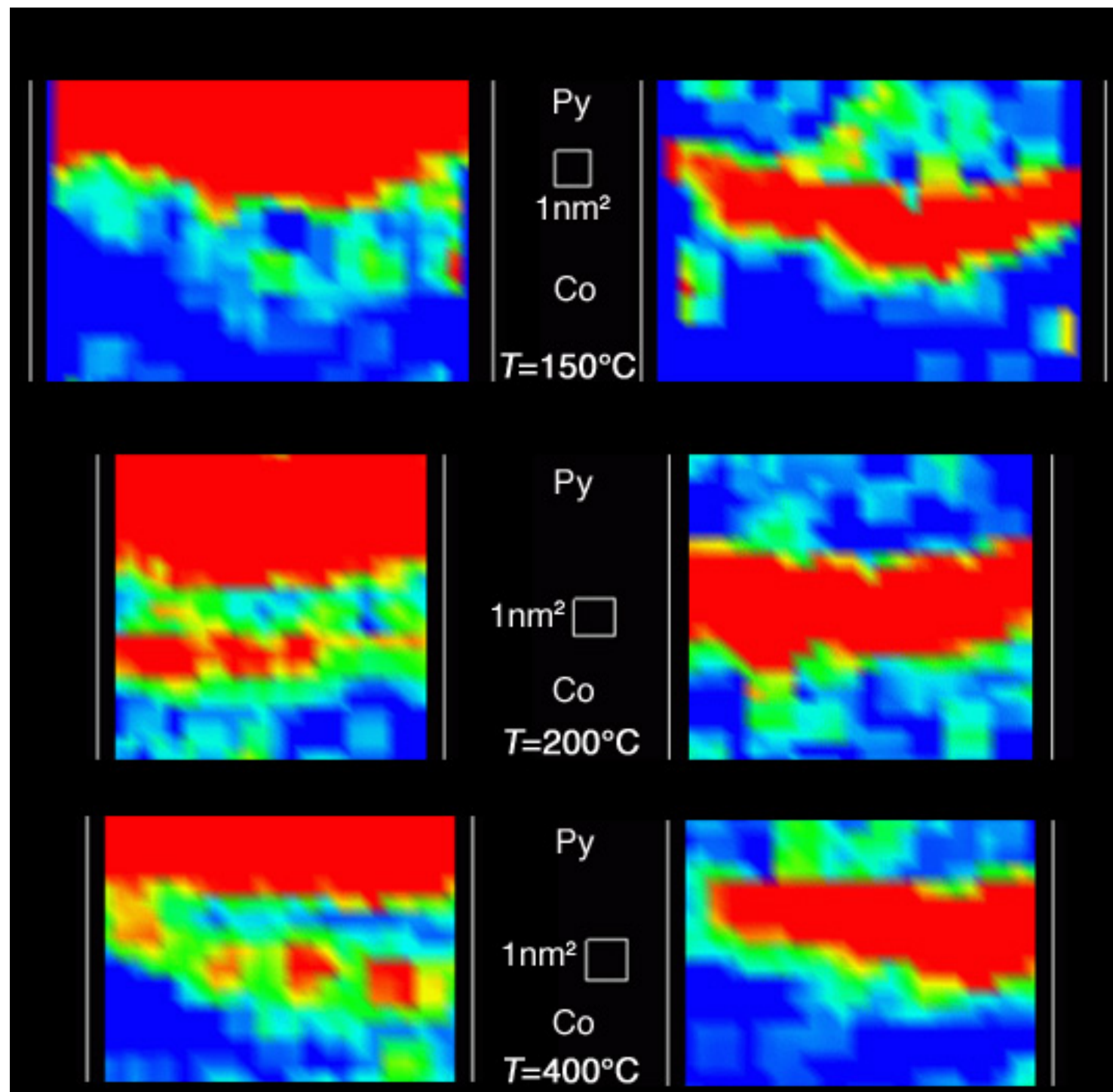

Fe concentration

\section{Al concentration}
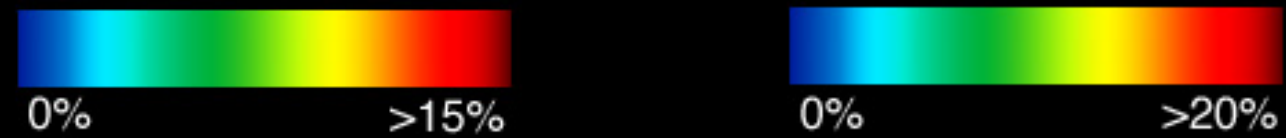

Figure 4.9: Two-dimensional $\mathrm{Al}$ and Fe composition maps of the barrier region after annealing at $T=150^{\circ} \mathrm{C}, T=200^{\circ} \mathrm{C}$ and $T=400^{\circ} \mathrm{C}$. The area of high Al content marks the position of the barrier. At its bottom interface (towards the Co layer) a thin layer of iron enrichment is located after annealing at temperatures of $T=200^{\circ} \mathrm{C}$ and above. Apart from statistical fluctuations the segregation layer appears to be continuous. 


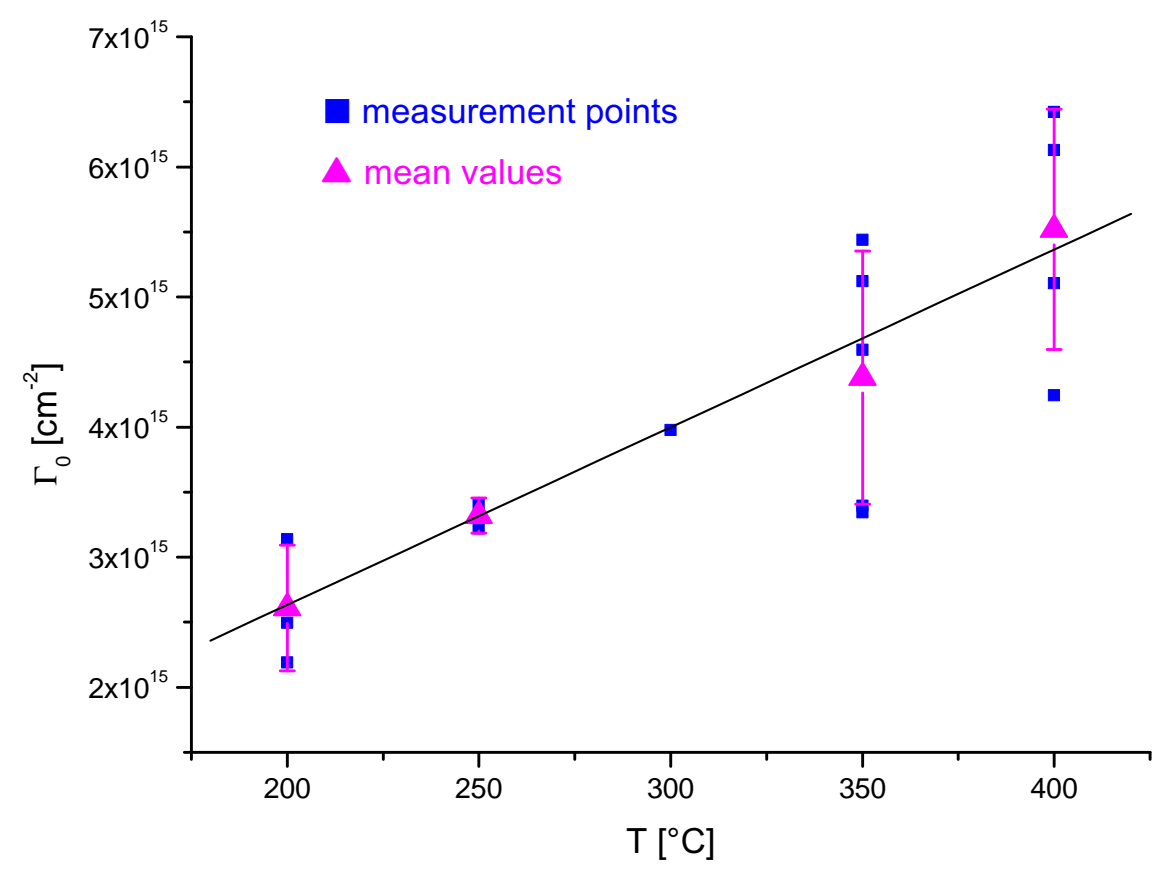

Figure 4.10: The Gibbsian interfacial excess of Fe atoms in dependence of the annealing temperature. The blue dots show individual measurements while the magenta triangles mark the mean values of the measurements.

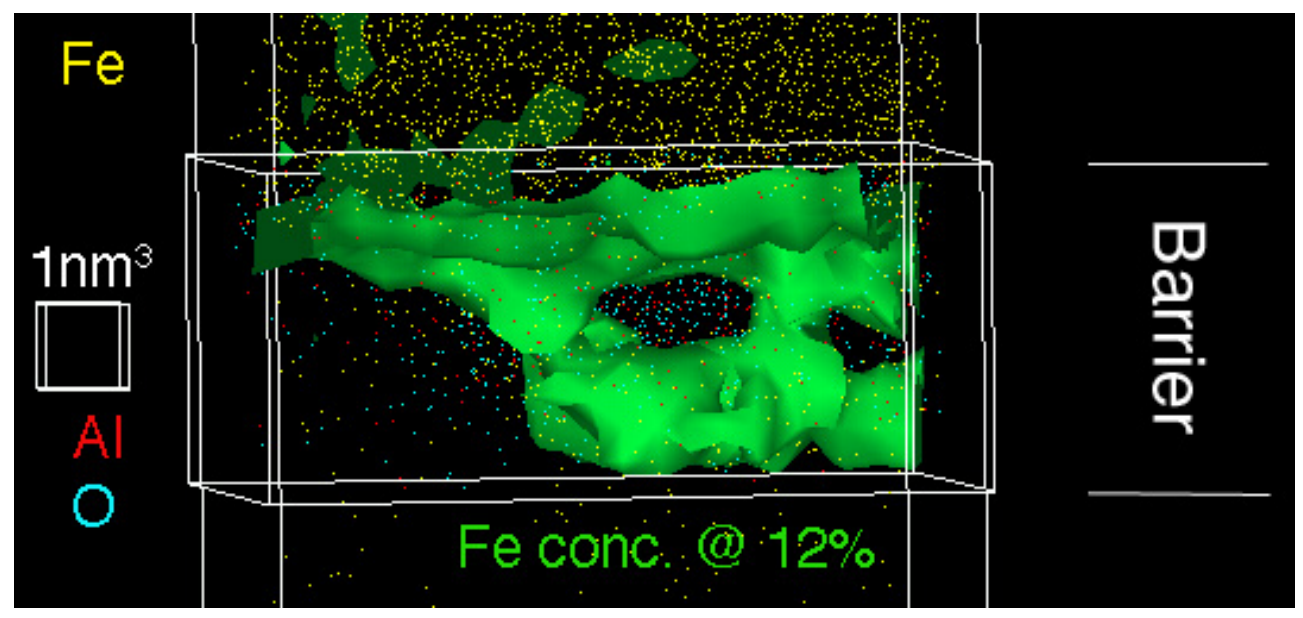

Figure 4.11: Iso-concentration surface at 12 at\% of Fe. This representation clearly shows the breakthrough channels of iron oxide. 
quantities of Co are found inside the Py and also quantities of $\mathrm{Ni}$ and Fe from the Permalloy layer are found inside the Co layer. There seems to be a dependence between the amount of Fe interface segregation and the amount of Co visible inside the Py layer. If the mass-spectrum shows a clear Co peak in the spectrum of the Co layer, the concentration profile shows a quiet low Fe peak at the barrier/Co interface (Fig. 4.12) and vice versa (Fig. 4.13). At annealing temperatures of about $T=500^{\circ} \mathrm{C}$ the rate of successful measurements decreases rapidly due to the degeneration of the sample structure: Only $5 \%$ of the samples could be measured successfully. In these cases $\mathrm{Al}$ atoms rest in their initial position while all other elements are completely redistributed within the stack. As it can be seen in the concentration profile in Fig. 4.14, O migrates into the electrodes. Presumably this effect of electrode oxidation makes the measurements more unstable and hence, decreases the rate of successful measurements.

The processes occurring in annealed states and their dependence on increasing annealing temperature can be summarized as follows:

1. Up to $T=150^{\circ} \mathrm{C}$, the setup is not influenced at all by the annealing. The system remains in as-prepared state.

2. From $T=200^{\circ} \mathrm{C}$ on, Fe interface segregation is observed. The intermixing of $\mathrm{Ni}$ and Fe within the barrier decreases, while the number of Fe atoms at the barrier/ Co interface increases.

3. Samples annealed at $T=300^{\circ} \mathrm{C}$ show breakthrough channels across the barrier structure enriched with Fe.

4. At $T=400^{\circ} \mathrm{C}$ the Fe segregation layer becomes more and more discontinuous. The number of Fe atoms at the interface increases. The intermixing of $\mathrm{Ni}$ and Fe within the barrier completely vanishes.

5. Samples annealed at $T=450^{\circ} \mathrm{C}$ show a certain amount of Co inside the Py layer. The quantity of Co inside Py correlates with the amount of Fe atoms at the barrier/ Co interface.

6. At $\mathrm{T}=500^{\circ} \mathrm{C}$ additionally to the occurrence of Co inside $\mathrm{Py}$, Fe and $\mathrm{Ni}$ atoms are detectable inside the Co layer. The Fe segregation between the barrier and the Co layer completely vanishes. Except for the Al atoms which formed the former barrier, all other atoms are completely redistributed within the whole stack. Only $5 \%$ of the samples can be measured successfully.

Normally, a certain annealing temperature is required to allow the migration of certain atoms by a volume diffusion mechanism. However, in some cases, a migration is possible at even lower annealing temperatures. This occurs whenever there are grain boundaries or any other kind of weak links within the structure and short circuit transport and segregation is possible. A measurement showing such a segregation of Co along a grain boundary inside the Py layer as presented in Fig. 4.15: this sample was annealed at $T=400^{\circ} \mathrm{C}$. This example shows that migration of atoms through the barrier may be accelerated by the presence of a grain boundary. 

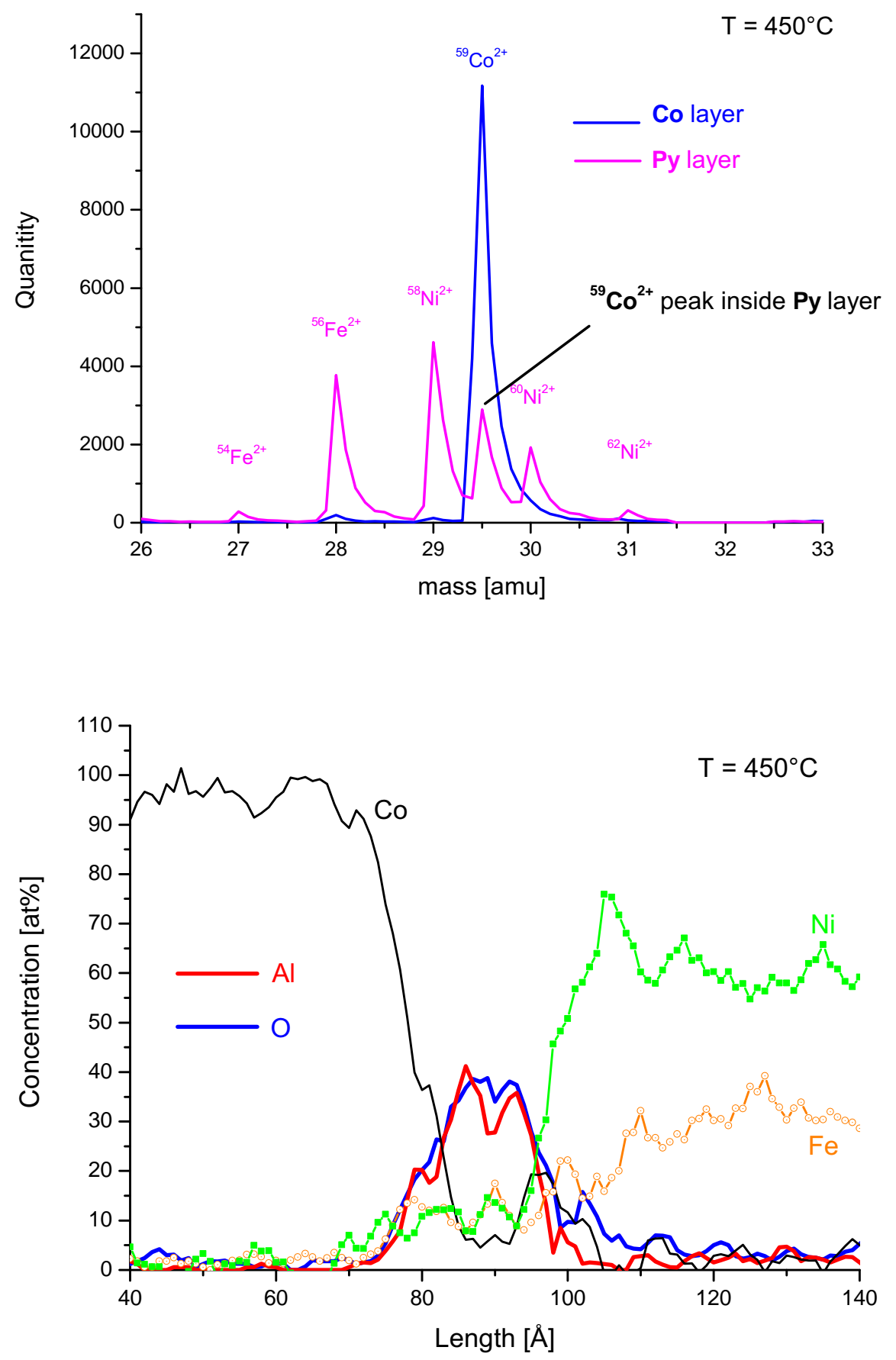

Figure 4.12: Mass spectrum and the corresponding concentration profile of a sample annealed at $T=450^{\circ} \mathrm{C}$. The Fe interface segregation has vanished while the amount of Co inside the Py layer has increased. 

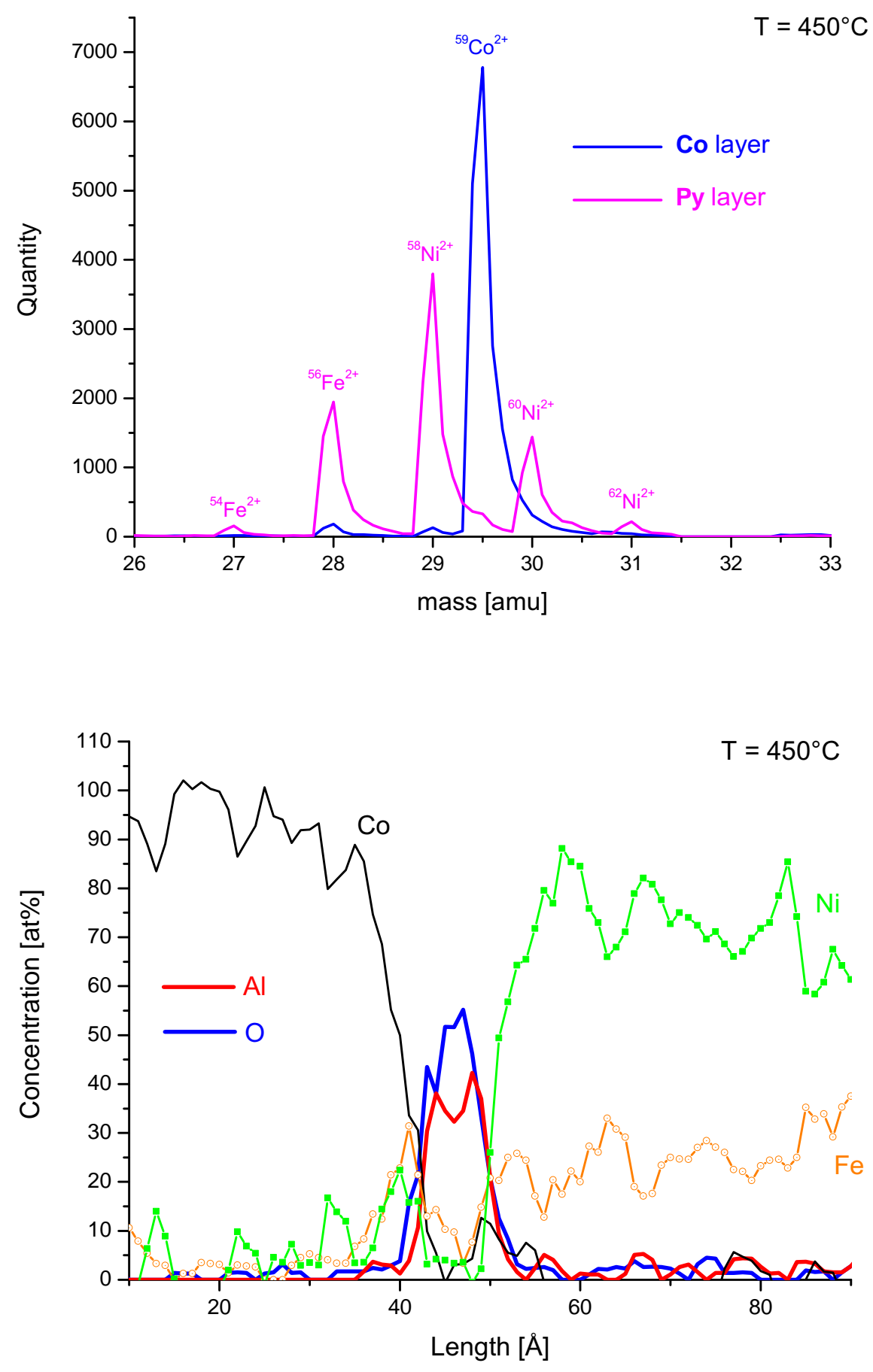

Figure 4.13: Mass spectrum and the corresponding concentration profile of a sample annealed at $T=450^{\circ} \mathrm{C}$. In comparison to Fig. 4.12, the Fe interface segregation is still present. There is no significant amount of Co inside the Py layer. 

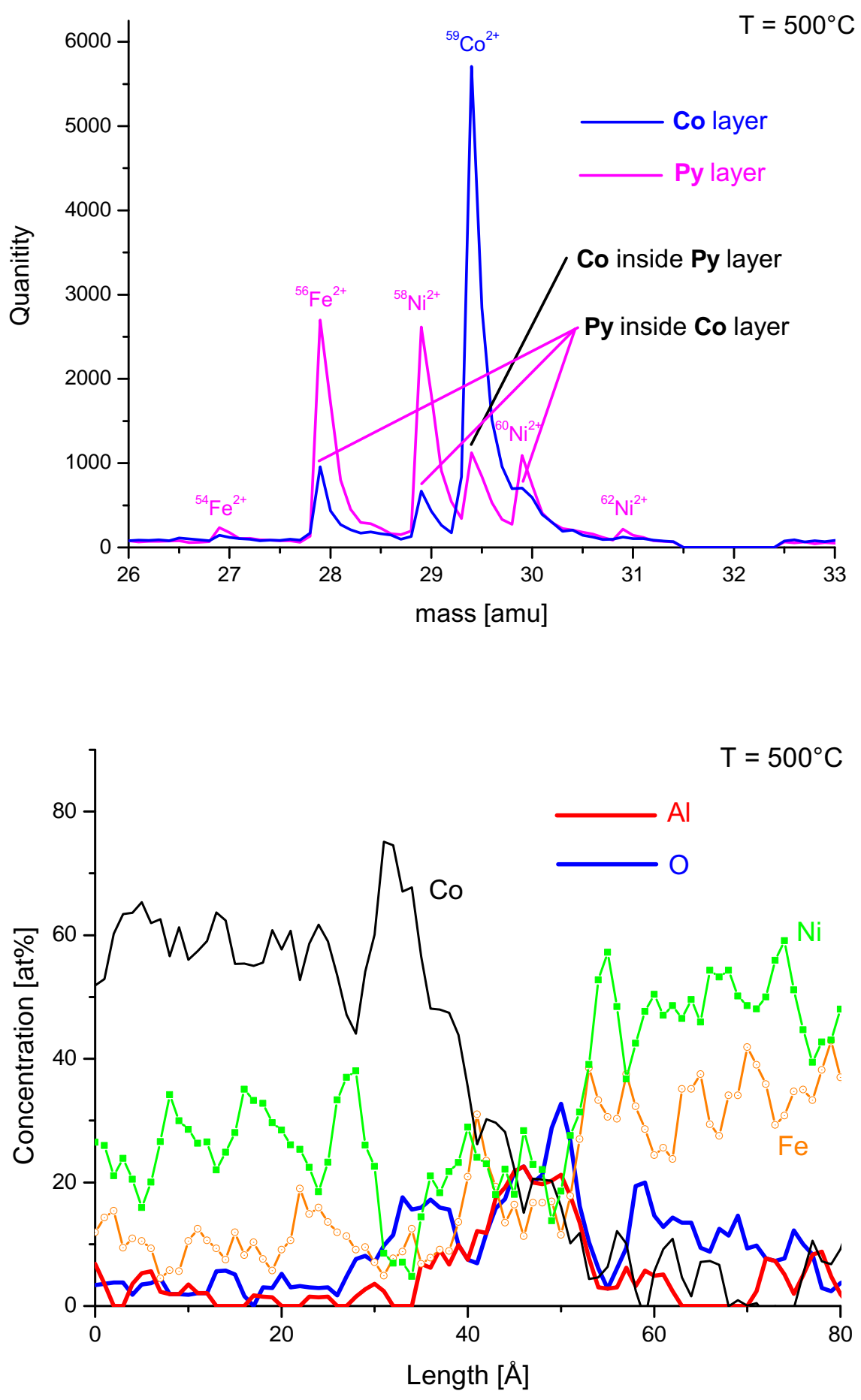

Figure 4.14: Mass spectrum and the corresponding concentration profile of a sample annealed at $T=500^{\circ} \mathrm{C}$. The Al remains in its initial position while the other elements are redistributed within the whole stack. 


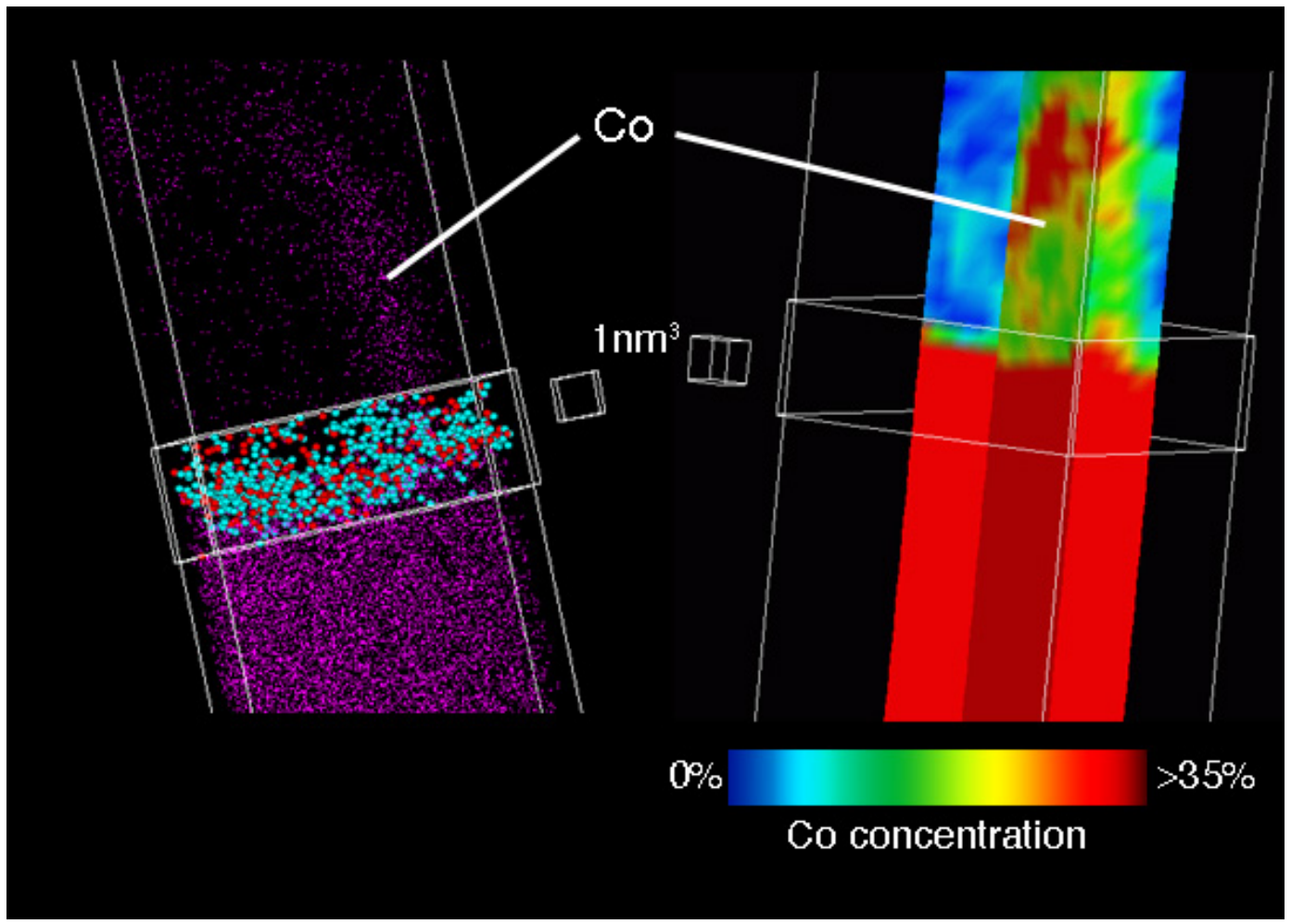

Figure 4.15: Grain boundary segregation of Co along a Py grain boundary after annealing at $T=400^{\circ} \mathrm{C}$. 


\subsection{TEM results}

The length scale in TAP measurements must be derived from the reconstruction parameters. As described in section 3.4.1, the reconstruction is based on the estimation of the curvature radius and the shaft angle of the sample. These parameters deviate from sample to sample, hence the reconstruction has to be done carefully for each measurement.

In order to confirm the derived thickness of the barrier and its surrounding layers, additional TEM analyses were performed. For that, planar samples were prepared simultaneously together with FIM tips in the sputter deposition chamber (see sections 3.1 .2 and 3.2). In order to guarantee the FIM tips' electron transparency, the final Co layer was deposited for only $30 \mathrm{~s}$, that corresponds to a layer thickness of $\sim 2 \mathrm{~nm}$. These samples were observed afterwards in a TEM system. Results are shown in Fig. 4.16. The thicknesses of the layers correspond to those derived by the TAP measurements. Therefore, one can assume the TAP reconstruction parameters to be adequate.

\subsection{SIMS results}

As it was shown in section 4.1.1, $\mathrm{Ni}$ and Fe have been detected within the oxide barrier. In order to prove these results and to be sure that this is not a measurement artefact of TAP at the non-conduction layer, complementary SIMS measurements were obtained. SIMS derives its data by time-of-flight measurements of sputtered atoms (see section 3.5.2). The SIMS profiles shown in Fig 4.17 show the results of measurements performed with samples where the "barriers" consist of either pure $\mathrm{Al}$ or $\mathrm{Al}_{2} \mathrm{O}_{3}$. The height of the curves of each element contains no direct information about the local content, since the different heights are influenced by severe matrix effects. Due to the fact that the deposited layers are relatively thin, these matrix effects occur quite often. Nevertheless the profiles show, that solution of $\mathrm{Ni}$ and $\mathrm{Fe}$ in the barrier only occurs, if oxygen is present. The barrier interface must be defined, where the height of the $\mathrm{Al}$ curve rises above that of the curve of Co. In the sample with pure $\mathrm{Al}$, neither $\mathrm{Ni}$ nor $\mathrm{Fe}$ is present there, while in the sample containing the oxide barrier, the amount of $\mathrm{Ni}$ and Fe rises. Hence, the results obtained by TAP

concerning the impurities of $\mathrm{Ni}$ and Fe inside the barrier are confirmed by the SIMS results

A SIMS measurement of an annealed sample at $T=400^{\circ} \mathrm{C}$ was also obtained. In this measurement, an additional Fe peak is detectable at the barrier/Co interface (see Fig 4.18) which proves the barrier/Co interface segregation independently.

\subsection{Electrical properties and TMR}

The electrical properties and the TMR of the planar samples were measured as described in section 3.1 .2 and 3.5.3. The samples were characterized by different 

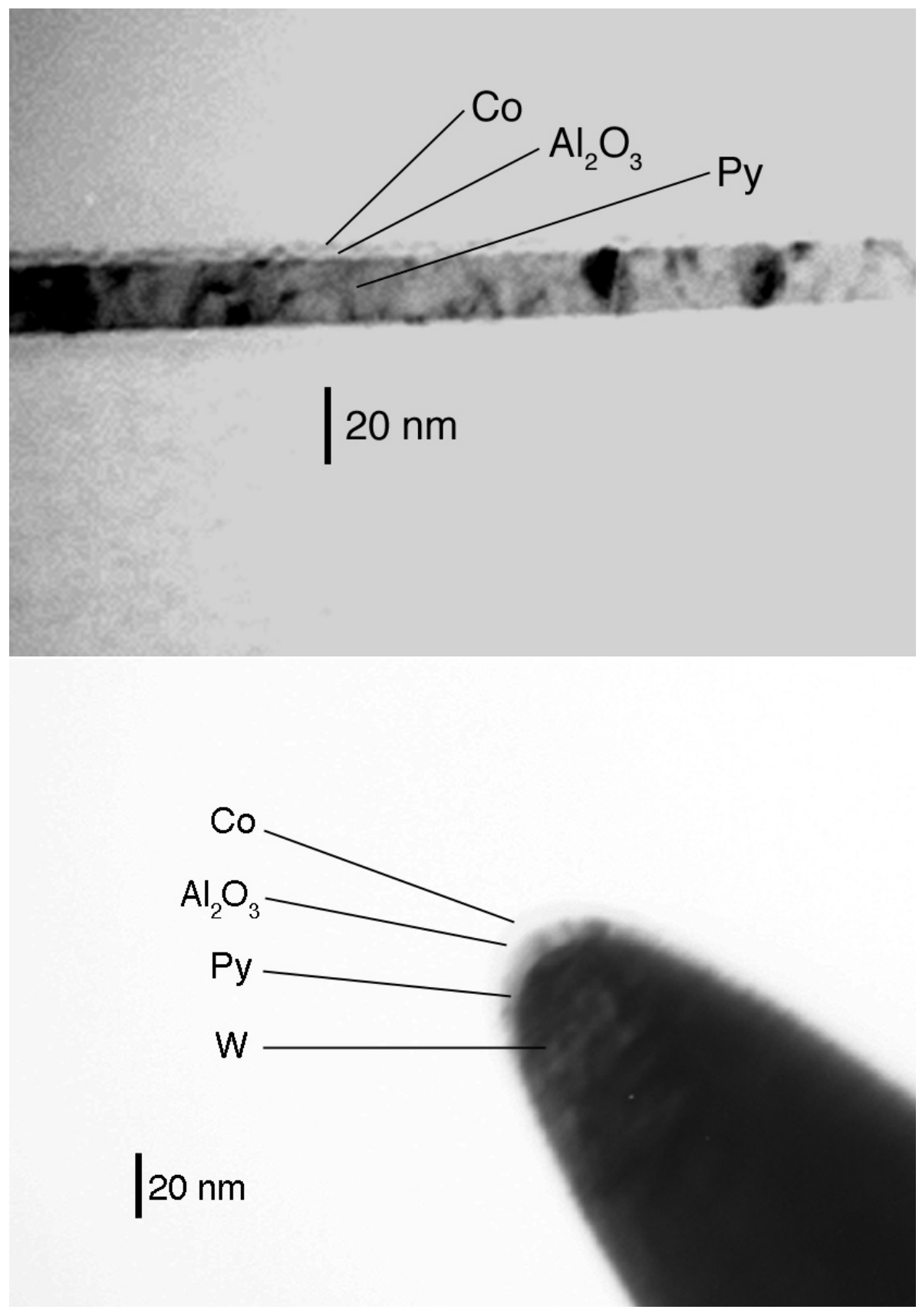

Figure 4.16: Images of a planar sample (above) and a FIM tip (bottom) observed in a TEM. One can identify the deposited layers directly. The tiny bright layer between the thick crystalline Py layer and the tiny dark Co is the $\mathrm{Al}_{2} \mathrm{O}_{3}$ barrier. The thicknesses correspond to those, derived by TAP measurements. 

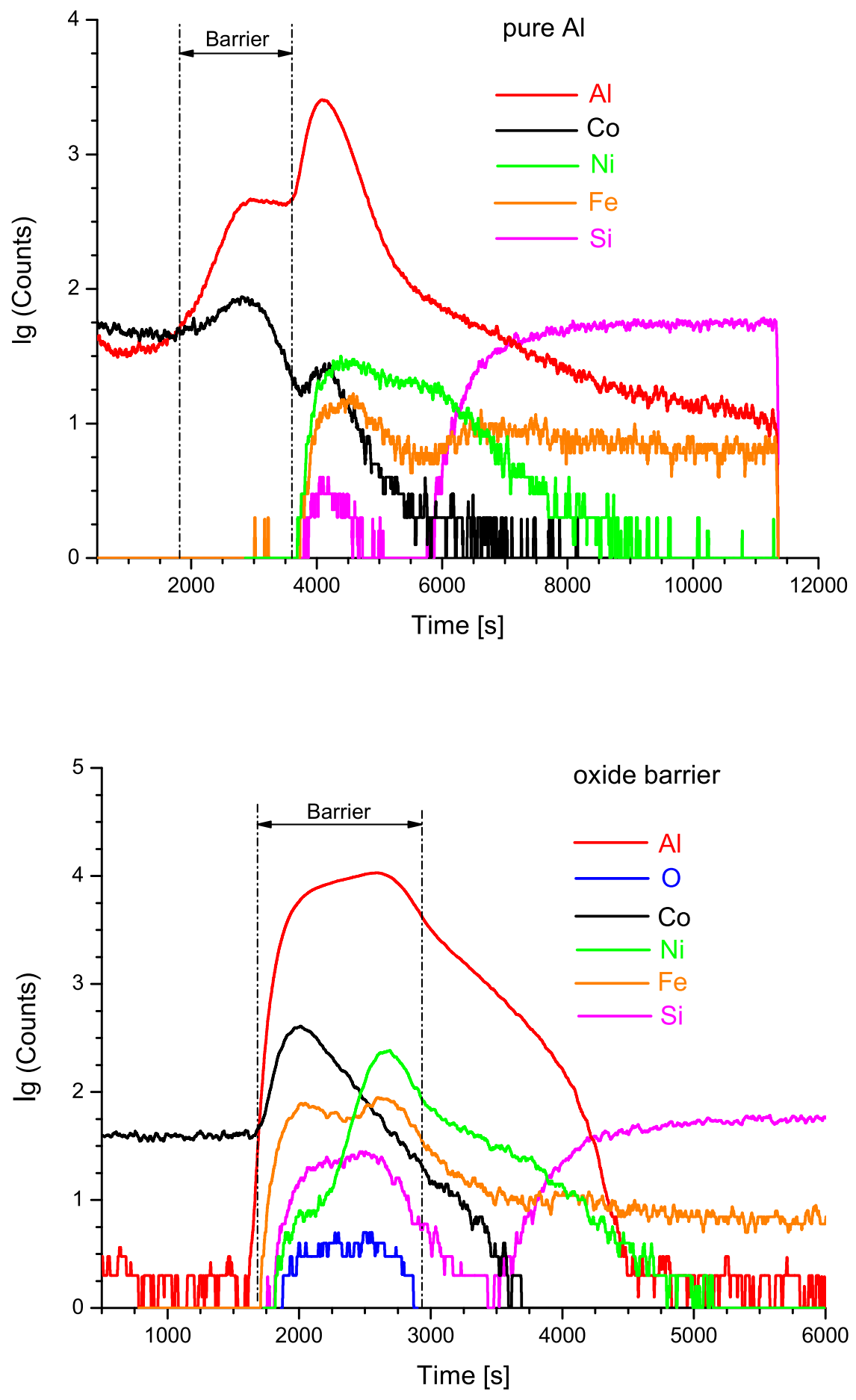

Figure 4.17: SIMS profiles of a barrier consisting of pure AI (up) and with an oxide barrier in annealed state (bottom). The SIMS data confirm the TAP results. 


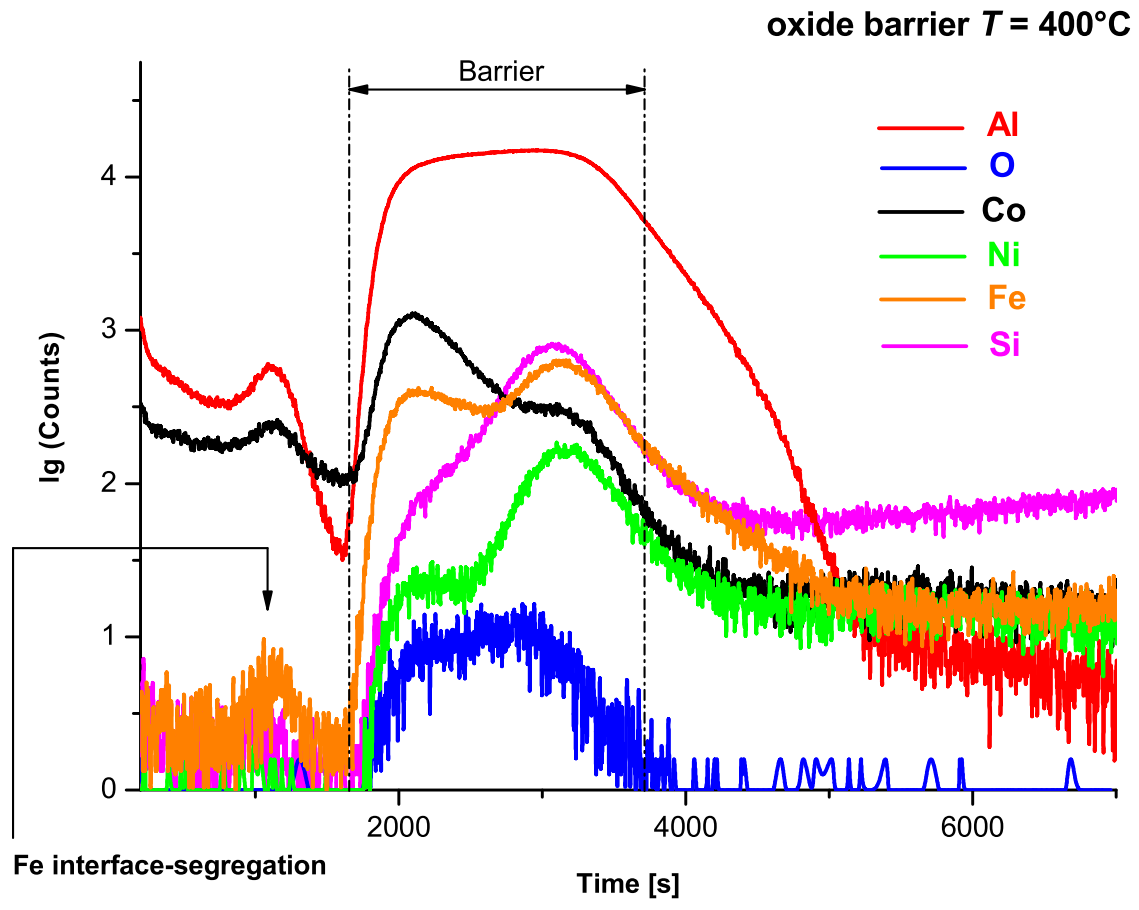

Figure 4.18: SIMS profile of a measurement with a barrier consisting of an oxide barrier annealed at $T=400^{\circ} \mathrm{C}$. Compared to the as-prepared state (see Fig. 4.17), an additional peak of $\mathrm{Fe}$ is visible at the barrier/ Co interface. 
Table 4.2: Overview of the samples used for electrical characterization

\begin{tabular}{lcl}
$\begin{array}{l}\text { Sample } \\
\text { No. }\end{array}$ & $\begin{array}{c}\text { deposition time } \\
\text { time }\end{array}$ & $\begin{array}{l}\text { expected barrier thickness } \\
\text { thickness }\end{array}$ \\
\hline 1 & $7.0 \mathrm{~min}$ & $2.0 \mathrm{~nm}$ \\
2 & $5.0 \mathrm{~min}$ & $1.5 \mathrm{~nm}$ \\
3 & $3.0 \mathrm{~min}$ & $1.0 \mathrm{~nm}$ \\
\hline
\end{tabular}

thicknesses of the $\mathrm{Al}_{2} \mathrm{O}_{3}$ barrier. The deposition was performed to produce thicknesses of $2.0 \mathrm{~nm}, 1.5 \mathrm{~nm}$ and $1.0 \mathrm{~nm}$. An overview is given in Tab. 4.2. All samples show magnetic properties as shown in Fig. 4.19. That means that in principle TMR is possible. The next step is to investigate whether the deposited $\mathrm{Al}_{2} \mathrm{O}_{3}$ barriers have properties of a tunnel-barrier. In a tunnel-barrier the $U / I$ characteristic curve is expected to follow a non-linear behavior while in a conventional ohmic resistor the behavior is linear. The samples 1 and 2 show a clear separation of the top and bottom electrode by the $\mathrm{Al}_{2} \mathrm{O}_{3}$ barrier which is represented by a non-linear behavior of the $U / I$ characteristic curve (see Fig. 4.20), that in principle allows a TMR effect. The $U / I$ characteristic curve of sample 3 shows a linear behavior. This suggests that the barrier does not has a closed structure and hence shortcuts occur and prevent the device from representing a real tunnel-barrier. This can be explained by the relatively low thickness 1 , according to the low deposition time.

TMR measurements were obtained for samples 1 and 2 . Only the sample with the largest thickness (sample 1) showed a measurable TMR effect. Sample 2 did not show a significant change in resistance during application of a magnetic field to the sample, although the $U / I$ characteristics indicates a closed barrier structure. The reason might be a relatively high roughness of the barrier. Sample 1 showed a TMR effect of $\sim 3.5 \ldots 4.5 \%$ (see Fig. 4.21). This relatively low value can also be explained by the high surface roughness, that has to be expected on samples prepared by ion beam deposition. The data points in the middle of the graph in Fig. 4.21 with TMR of $1 \%$ at 0 Oe occur due to the low difference in magnetization of the two electrodes. The change in magnetization of the electrodes occurs only with a very short delay during variation of the external magnetic field. In order to avoid this effect, in technical applications one electrode is "pinned" by an additional ferromagnet attached on top of one of the electrodes. This makes one electrode change its magnetization after a higher delay compared to the other electrode.

The $U / I$ characteristic curves of the samples were obtained at several locations of the samples and then adapted to the model given by Brinkman et al. (1970) as described in section 2.2. The results are given in Tab. 4.3. The investigated barrier structures deviate from the "ideal barrier" values in several ways: Especially the asymmetry of the barriers is partially much higher than $0 \mathrm{eV}$, and all values fluctuate with the position on the sample's surface. This can also be interpreted as a

\footnotetext{
${ }^{1}$ expected thickness $1.0 \mathrm{~nm}$, compare to Tab. 4.2
} 

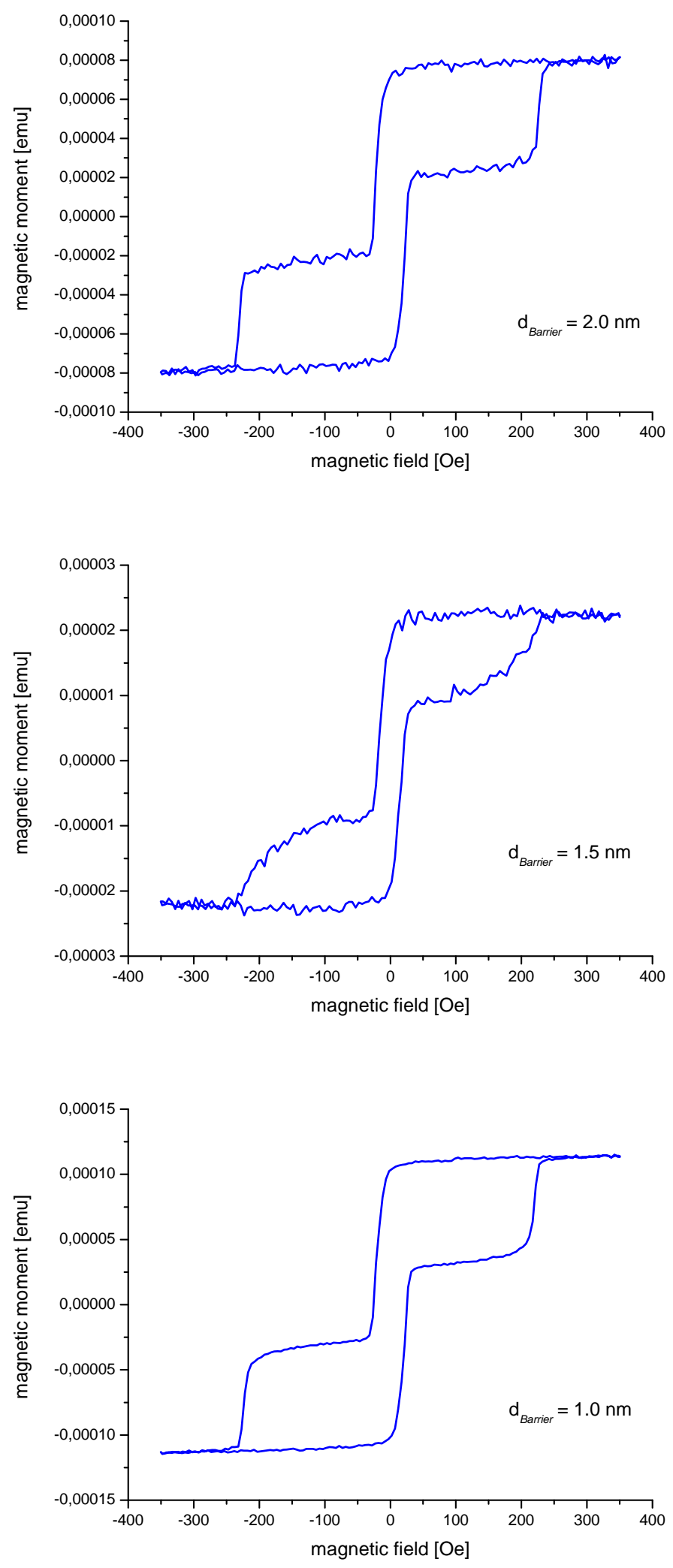

Figure 4.19: Characteristic magnetic curves for each of the three samples. All samples show ferromagnetic properties. 

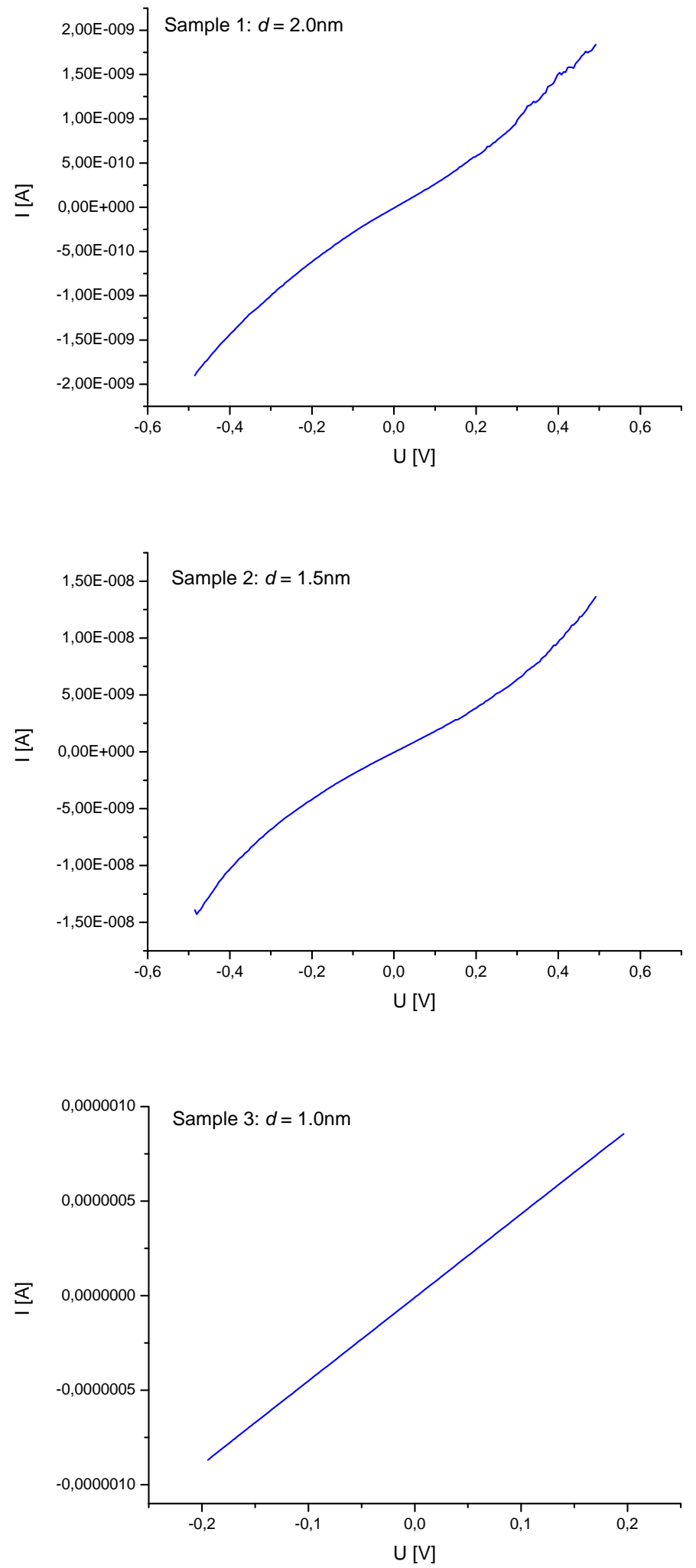

Figure 4.20: $U / I$ characteristic curves for each of the measured samples. The sample with the lowest thickness $(d=1 \mathrm{~nm})$ shows a linear behavior that does not represent a tunnel-barrier but a conventional ohmic resistor. 


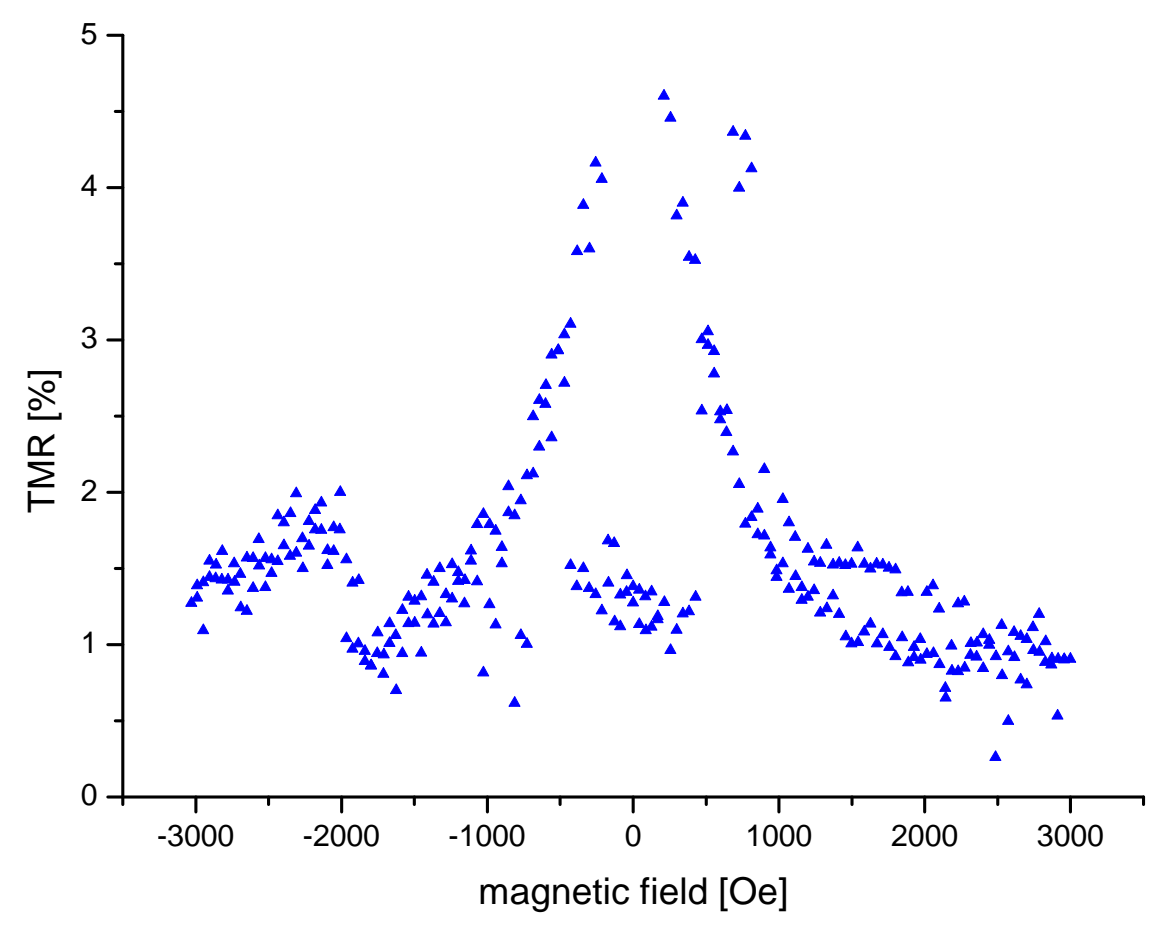

Figure 4.21: TMR characteristic curve of sample 1, the only sample which showed a significant TMR effect. The values of $1 \%$ TMR at 0 Oe occur due to the low difference in magnetization of the electrodes. Pinning of one of the electrodes avoids this effect.

Table 4.3: Overview of the results of the fit to the $U / I$ characteristic curves according to the model by Brinkman et al. (1970) in comparison to an ideal barrier.

\begin{tabular}{lccc}
$\begin{array}{l}\text { Sample } \\
\text { No. }\end{array}$ & $\begin{array}{c}\text { Thickness } \\
{[\AA]}\end{array}$ & $\begin{array}{c}\text { Asymmetry } \\
{[\mathrm{eV}]}\end{array}$ & $\begin{array}{c}\text { Barrier Hight } \\
{[\mathrm{eV}]}\end{array}$ \\
\hline 1 & 29.74 & 1.50 & 1.00 \\
1 & 26.98 & 0.93 & 1.30 \\
1 & 26.56 & 0.11 & 1.35 \\
\hline 2 & 18.52 & 5.18 & 2.75 \\
2 & 14.40 & 21.18 & 6.05 \\
2 & 24.28 & 0.52 & 1.97 \\
\hline \hline ideal barrier & 18.00 & 0.00 & 3.00 \\
\hline
\end{tabular}


sign of a relatively high barrier roughness. Nevertheless, the deposited stack with a barrier thickness identical to that of the TAP specimens has TMR properties and in consequence the results derived by TAP may be transfered to TMR devices in general. 


\section{Chapter 5}

\section{Discussion}

$\mathrm{Py} / \mathrm{Al}_{2} \mathrm{O}_{3} /$ Co spin valve structures produced by ion beam sputtering of metals in an oxygen atmosphere or by subsequent natural oxidation, have been chemically characterized by 3D atom probe tomography (TAP). The experimental results presented, demonstrate that oxide tunnel barriers can be field evaporated in a sufficiently controlled manner to permit a reasonable chemical analysis and reconstruction. This conclusion is supported by the quantitative data, which yielded the correct stoichiometry and a reasonable dependence of the determined oxygen content on the conditions of formation of the oxide layer.

At first sight, the possibility of investigating isolating ceramics by field ion microscopy is rather astounding. However, one has to remember the successful analysis of small, oxidic precipitates embedded in a metallic matrix, which has been reported by Rüsing et al. (2000), and Kluthe et al. (2002) in recent years. According to the present experience obtained in this work, measurements were successful only up to an oxide thickness of $2.5 \mathrm{~nm}$, that corresponds to a thicknesses chosen in spin valve devices to permit a certain amount of conductivity via electron tunneling. Thus, the most important limitation of the material with respect to field ion microscopy the low conductivity is circumvented in nano-scaled devices. The results show a noteworthy parallelism to scanning tunneling microscopy. Although also the latter method is in principle restricted to conductors, thin surface films of non-conductive organic materials on metallic substrates can nevertheless be investigated.

\section{$5.1 \quad$ As-prepared state}

One of the aims of this work has been to test the feasibility of the atom probe technique to analyze oxidic tunnel barriers. However, a few definite statements to the chemistry of the oxidic barriers are already possible. Regarding in-situ oxidation, $\mathrm{Al}_{2} \mathrm{O}_{3}$ barriers with the right stoichiometry are only achieved with a partial pressure of at least $3 \cdot 10^{-5} \mathrm{mbar}$. On the other hand, a pressure up to $1 \cdot 10^{-4} \mathrm{mbar}$ does not lead to a partial oxidation of the ferromagnetic electrodes. The natural subsequent 
Discussion

oxidation of the already deposited Al layer at oxygen pressures up to $1 \cdot 10^{-4}$ mbar produces severely hypo-stoichiometric oxides. This result is in good agreement with the observations described in Moon et al. (2002) and furthermore with a recent quantitative study by Jeurgens et al. (2002) on the oxidation rate of coarse grained Al. Using a slightly lower partial pressure of oxygen but an elevated temperature of $373 \mathrm{~K}$, the latter authors determined an apparent maximum oxidation depth of $0.4 \mathrm{~nm}$, which is much less than the thickness of the initial Al in our metallic trilayer. Thus, it is no surprise to detect a severe deficit of oxygen. However, as revealed by the nano-analysis, this oxygen is distributed almost homogeneously throughout the barrier which may be caused by the nano-crystallinity of the Al layers studied here. As a consequence, the one step natural oxidation is not applicable for the preparation of TMR oxide barriers thicknesses of the order of $1.5 \mathrm{~nm}$.

A quite remarkable feature of the oxide barriers produced is an appreciable content of the components of the Py electrode. This effect is represented by a mixing of $\mathrm{Ni}$ and Fe into the barrier. Measurements with a 'barrier' consisting of pure Al do not show such an intermixing. In that case the interface is quiet sharp with a width of $\sim 5 \AA$. These results are confirmed by Secondary Ion Mass Spectrometry (SIMS) which shows the same behavior of $\mathrm{Ni}$ and $\mathrm{Fe}$ as in the TAP analysis.

One might expect this phenomenon to occur due to ion-mixing during the deposition: the deposited Al atoms have enough energy to provide an ion-mixing at the tips surface so that $\mathrm{Fe}$ and $\mathrm{Ni}$ atoms from the lower Py layer are mixed up with the $\mathrm{Al}_{2} \mathrm{O}_{3}$. To clarify this effect, a SRIM20031 simulation has been performed (Ziegler et al., 1985). First the energy of the Al atoms sputtered from the target by the $\mathrm{Ar}$ beam (see section 3.2 was calculated. Knowing this energy, the application of the $\mathrm{Al}$ atoms with the in the first step calculated energy to the $\sim 5 \AA$ thick $\mathrm{Al}_{2} \mathrm{O}_{3}$ layer was simulated. The results do not show any significant intermixing affected by the incoming $\mathrm{Al}$ atoms.

The reason for the intermixing of the Py elements might result from the properties of the $\mathrm{Al}_{2} \mathrm{O}_{3}$ barrier: $\mathrm{Al}_{2} \mathrm{O}_{3}$ is usually assumed to have an amorphous structure (e.g. Parkin et al. (1999a)), but there is also the possibility that it has a nano-crystalline structure with such tiny grains, that it appears in all standard analysis methods as an amorphous layer. Assuming that $\mathrm{Al}_{2} \mathrm{O}_{3}$ has nano-crystalline properties, the intermixing of $\mathrm{Ni}$ and $\mathrm{Fe}$ can be explained by grain boundary segregation, where $\mathrm{Ni}$ and $\mathrm{Fe}$ is aligned along the $\mathrm{Al}_{2} \mathrm{O}_{3}$ grain boundaries. Thus the the intermixing of $\mathrm{Ni}$ and Fe inside the $\mathrm{Al}_{2} \mathrm{O}_{3}$ barrier observed in this work indeed suggest that a nano-crystalline structure of the $\mathrm{Al}_{2} \mathrm{O}_{3}$ barrier is present.

\footnotetext{
${ }^{1}$ The $\mathbf{S}$ topping and $\mathbf{R}$ ange of $\mathbf{I}$ ons in $\mathbf{M}$ atter, a free software for simulation of stopping and range of ions in matter. It is a further development of the TRIM software (The $\operatorname{Tr}$ ansport of $\mathbf{I}$ ons in $\mathbf{M}$ atter)
} 


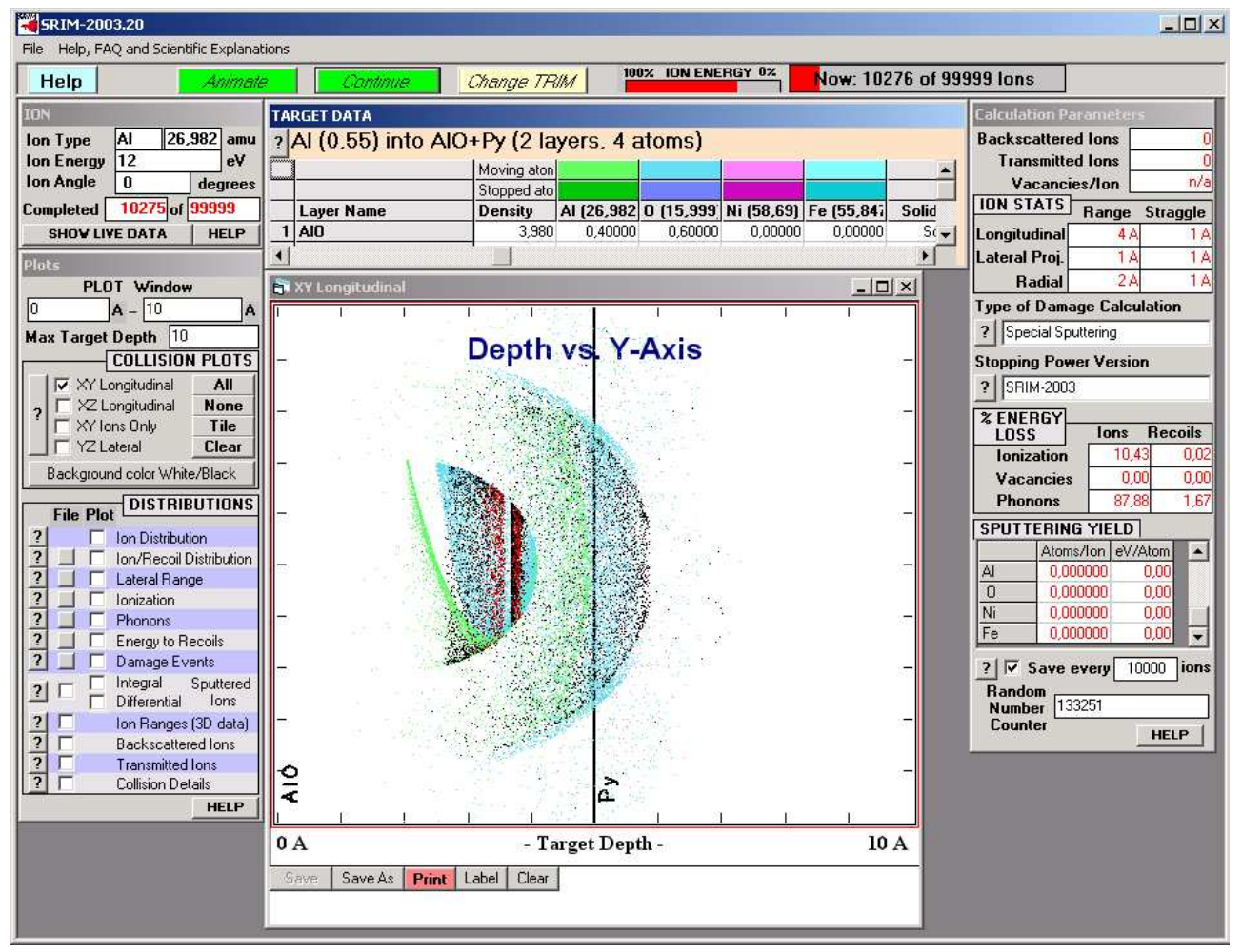

Figure 5.1: SRIM simulation window. The values in the lower right show that no sputter yield is expected according to the simulation. 


\subsection{Annealed state}

\subsubsection{Phenomenological description}

After the as-prepared samples were successfully analyzed, they were annealed at temperatures from $T=150^{\circ} \mathrm{C}$ up to $T=500^{\circ} \mathrm{C}$ and afterwards investigated by TAP. The observed thermally induced reactions on a $\mathrm{Co} / \mathrm{Al}_{2} \mathrm{O}_{3} / \mathrm{Py}$ tri-layer seen in these measurements indicate two mechanisms by which the TMR amplitude may be optimized applying moderate heat treatments. Furthermore the analysis permits a better understanding of the degradation of desired properties at higher temperatures. In spite of the high stability and therefore low diffusivity of the aluminum oxide barrier, appreciable diffusion of electrode material takes place across the barrier even at low temperatures without damaging the clear layer structure. A similar effect has been observed recently for Co/ Cu/ Py GMR systems by Schleiwies et al. (2001). Segregation at the electrode interfaces may improve the spin polarization if suitable electrode components are selected. The maximum of TMR at approximately $240^{\circ} \mathrm{C}$ shown by Sousa et al. (1999) has been observed in a different stack system, but may be explained by the same kind of interface segregation transfered to the results presented in this work: the transport of ferromagnetic material to a different region of the stack, in case of the present results $\mathrm{Fe}$, changes the magnetic properties resulting in an improvement of the TMR of the $\mathrm{Py} / \mathrm{Al}_{2} \mathrm{O}_{3} / \mathrm{Co} \mathrm{TMR}$ system to the barrier/Co interface. At higher temperatures $\left(T \approx 300^{\circ} \mathrm{C}\right)$ metallic species are locally enriched inside the barrier, forming impurity channels of different conductivity, that introduce weak links, which may explain the cascade-like electrical failure described in Schmalhorst et al. (2000).

At annealing temperatures of more than $T=400^{\circ} \mathrm{C}$, the system gets more and more intermixed. While at $T=450^{\circ} \mathrm{C}$ only Co diffusion is observed in some of the samples, at $T=500^{\circ} \mathrm{C}$ all elements forming the electrodes show diffusion throughout the whole stack.

\subsubsection{Diffusion processes}

Little is known about the diffusion processes and solubility of metals in $\mathrm{Al}_{2} \mathrm{O}_{3}$. Some information is available from the online database provided by the National Institute of Materials and Science (NIMS / Japan), which gives an overview of all relevant diffusion data reported in literature on that topic. The plot shown in Fig. 5.2 is calculated using the data of the NIMS database, where the values for the activation energy $Q=333 \mathrm{~kJ} / \mathrm{mol}$ and the pre-factor $D_{0}=6.2 \cdot 10^{-9} \mathrm{~m}^{2} / \mathrm{s}$ are given for $\mathrm{Co}$ diffusion in $\mathrm{Al}_{2} \mathrm{O}_{3}$. No data for $\mathrm{Fe}$ and $\mathrm{Ni}$ are available; the values given for Co are assumed as an approximation for $\mathrm{Fe}$ and $\mathrm{Ni}$, using the fact that these elements are direct neighbors in the periodic system of the elements and might have similar properties. In an Arrhenius plot the slope of the graph gives the activation energy $Q$ over $R$. The diffusion constant depends on the temperature: the Arrhenius 


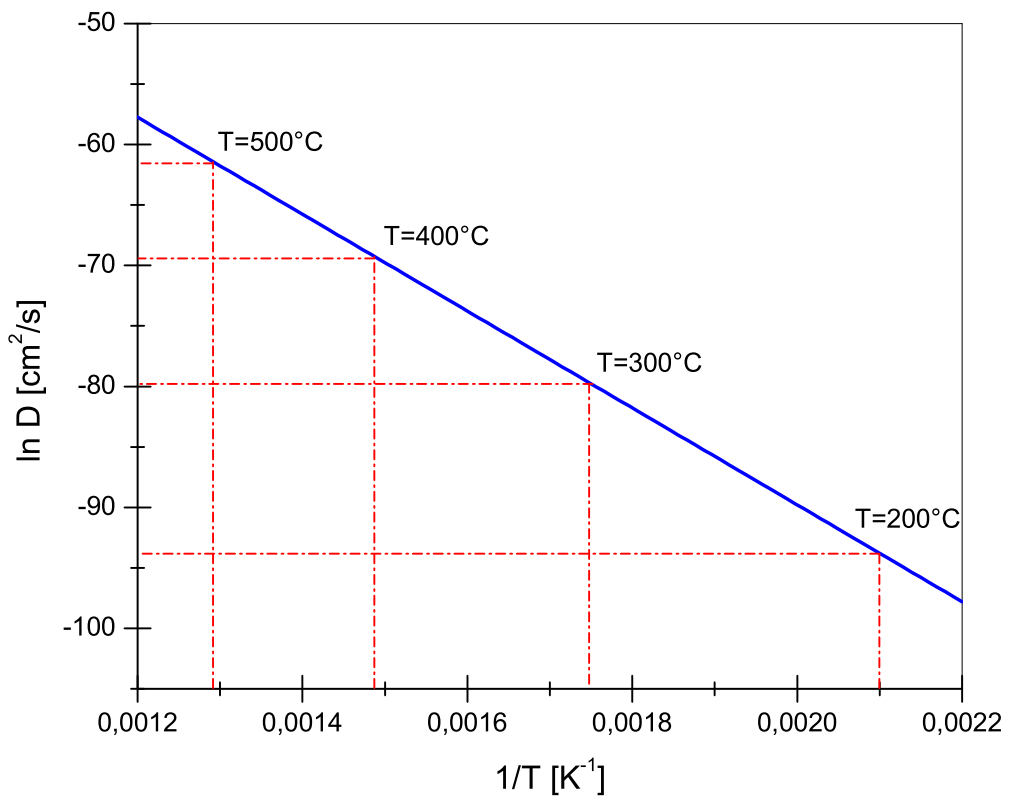

Figure 5.2: Arrhenius plot of Co diffusing into $\mathrm{Al}_{2} \mathrm{O}_{3}$ based on the data provided by the National Institute of Materials and Science (NIMS) for the temperature range of interest in this work $\left(Q=333 \mathrm{~kJ} / \mathrm{mol}^{-1}, D_{0}=6.2 \cdot 10^{-9} \mathrm{~m}^{2} \mathrm{~s}^{-1}\right.$.)

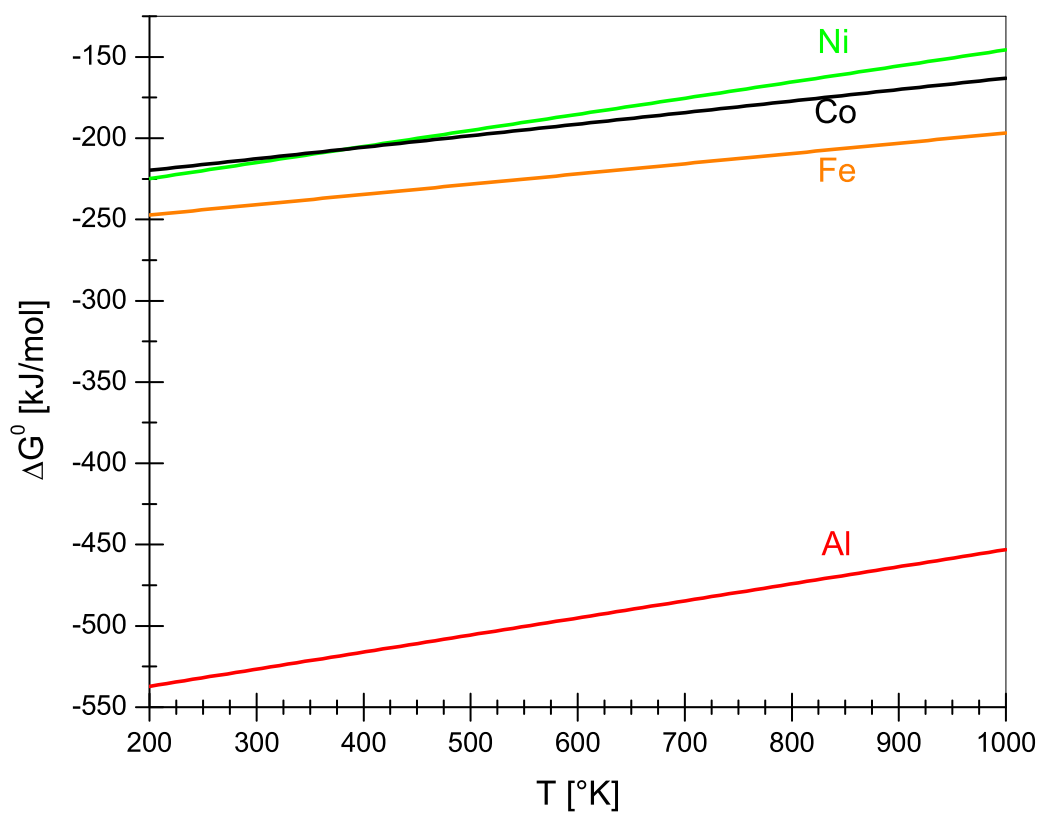

Figure 5.3: Ellingham diagram of the elements involved in the investigated setup. One recognizes that the formation free energy $\Delta G_{0}$ of $\mathrm{Al}_{2} \mathrm{O}-3$ is distinctively higher compared to the other elements. 
equation, including the general gas constant $R=8.31451 \mathrm{~J} / \mathrm{K}^{-1} \mathrm{col}^{-1}$, is

$$
D=D_{0} \cdot \exp \left(-\frac{Q}{R T}\right)
$$

or

$$
\ln D=-\frac{Q}{R} \cdot \frac{1}{T}+\ln D_{0}
$$

By Eq. 5.1 the diffusion constant $D$ can be calculated for every temperature. From the knowledge of $D$, one can derive the diffusion flux density $\vec{j}$ using the first Fick's law

$$
\vec{j}=-D \cdot \vec{\nabla} c
$$

where $c$ is the concentration and $\nabla c$ the concentration gradient. In case of a layer geometry a one-dimensional representation is sufficient. Furthermore a linear comparison variation across the barrier is assumed in steady state. Thus one gets

$$
\vec{\nabla} c=\frac{\partial c}{\partial x}=\frac{\eta}{d \cdot \Omega}
$$

where $\eta=0 \ldots 1$ is the solubility of the diffusing elements, $d$ the thickness the barrier thickness $(\sim 2 \mathrm{~nm})$, and $\Omega$ the average atomic volume of the matrix elements. In the case discussed in this work the matrix consists of $\mathrm{Al}$ and $\mathrm{O}$ atoms. A value for the atomic volume can be calculated from the density of $\mathrm{Al}_{2} \mathrm{O}_{3}: \Omega=8.51 \cdot 10^{-24} \mathrm{~cm}^{3}$. Because there is no exact information available concerning the solubility $\eta$ of the elements in $\mathrm{Al}_{2} \mathrm{O}_{3}$, a maximum of 1 is assumed, i.e. the whole amount of $\mathrm{Fe}, \mathrm{Ni}$ or Co is supposed to be soluted in $\mathrm{Al}_{2} \mathrm{O}_{3}$, yielding an upper limit for the theoretical diffusion flux density $\vec{j}$. Eq. 5.4 can be written in the form

$$
\vec{\nabla} c=\frac{\partial c}{\partial x}=\frac{1}{2 \cdot 10^{-7} \mathrm{~cm} \cdot 8.51^{-24} \mathrm{~cm}^{3}} .
$$

The diffusing elements $\mathrm{Fe}, \mathrm{Ni}$ and $\mathrm{Co}$ are assumed not to be oxidized by the quantity of $\mathrm{O}$ inside the barrier since the formation free energy $\Delta G_{0}$ of $\mathrm{Al}_{2} \mathrm{O}_{3}$ is distinctively high compared to the other elements involved in the system as seen in the Ellingham diagram in Fig. 5.3 that has been calculated using the data of Fromm and Gebhardt (1976). In other words, since Al has the highest affinity to oxygen, the oxygen is assumed to be totally bonded to the Al atoms.

The experimental $\vec{j}$ is derived directly by counting the segregated atoms divided by the reaction time (identical with the annealing time of $10 \mathrm{~min}$ ). The results of the calculations are given in Tab. 5.1. Since only data for Co diffusion in $\mathrm{Al}_{2} \mathrm{O}_{3}$ is available, these data were used for comparison in the case of $\mathrm{Ni}$ and $\mathrm{Fe}$, too. The results show a significant deviation of the experimental data of the diffusion flux density from the theoretical expectation (Eq. 5.3 and Eq. 5.4). This result suggests that volume diffusion can be excluded. Another possibility is the diffusion along grain boundaries or any other kind of defects. In grain boundary diffusion, the activation energy $Q$ is typically about one half of the activation energy necessary 
Table 5.1: Overview of the theoretically expected and experimentally derived diffusion data of different elements through the $\mathrm{Al}_{2} \mathrm{O}_{3}$ barrier.

\begin{tabular}{|c|c|c|c|c|c|}
\hline Element & $\begin{array}{c}\mathrm{T} \\
{ }^{\circ} \mathrm{C}\end{array}$ & $\begin{array}{c}D_{(t h r)} \\
{\left[\mathrm{cm}^{2} \mathrm{~s}^{-1}\right]}\end{array}$ & $\begin{array}{c}j_{(t h r)} \\
{\left[\mathrm{cm}^{-2} \mathrm{~s}^{-1}\right]}\end{array}$ & $\begin{array}{c}j_{(e x p)} \\
{\left[\mathrm{cm}^{-2} \mathrm{~S}^{-1}\right]}\end{array}$ & $\begin{array}{l}\text { location of } \\
\text { diffused elements }\end{array}$ \\
\hline $\mathrm{Fe}$ & 200 & $1.04 \cdot 10^{-41}$ & $6.11 \cdot 10^{-12}$ & $\begin{array}{l}7.27 \cdot 10^{+12} \\
5.38 \cdot 10^{+12} \\
6.78 \cdot 10^{+12}\end{array}$ & barrier/Co interface \\
\hline $\mathrm{Fe}$ & 250 & $3.41 \cdot 10^{-38}$ & $2.00 \cdot 10^{-8}$ & $\begin{array}{l}8.50 \cdot 10^{+12} \\
8.57 \cdot 10^{+12}\end{array}$ & barrier/Co interface \\
\hline $\mathrm{Fe}$ & 300 & $2.72 \cdot 10^{-35}$ & $1.60 \cdot 10^{-5}$ & $9.90 \cdot 10^{+12}$ & barrier/ Co interface \\
\hline $\mathrm{Fe}$ & 350 & $7.44 \cdot 10^{-33}$ & $4.37 \cdot 10^{-3}$ & $\begin{array}{l}8.88 \cdot 10^{+12} \\
9.02 \cdot 10^{+12} \\
8.40 \cdot 10^{+12} \\
9.93 \cdot 10^{+12}\end{array}$ & barrier/Co interface \\
\hline $\mathrm{Fe}$ & 400 & $8.83 \cdot 10^{-31}$ & $5.19 \cdot 10^{-1}$ & $\begin{array}{l}1.10 \cdot 10^{+13} \\
1.43 \cdot 10^{+13} \\
1.49 \cdot 10^{+13}\end{array}$ & barrier/Co interface \\
\hline $\mathrm{Fe}$ & 450 & $5.41 \cdot 10^{-29}$ & $3.18 \cdot 10^{+1}$ & $\begin{array}{l}1.18 \cdot 10^{+13} \\
1.54 \cdot 10^{+13}\end{array}$ & barrier/Co interface \\
\hline $\begin{array}{l}\mathrm{Fe} \\
\mathrm{Co} \\
\mathrm{Ni}\end{array}$ & 500 & $1.95 \cdot 10^{-27}$ & $1.15 \cdot 10^{+3}$ & $\begin{array}{l}3.42 \cdot 10^{+12} \\
5.77 \cdot 10^{+12} \\
9.00 \cdot 10^{+12}\end{array}$ & opposite electrode \\
\hline
\end{tabular}




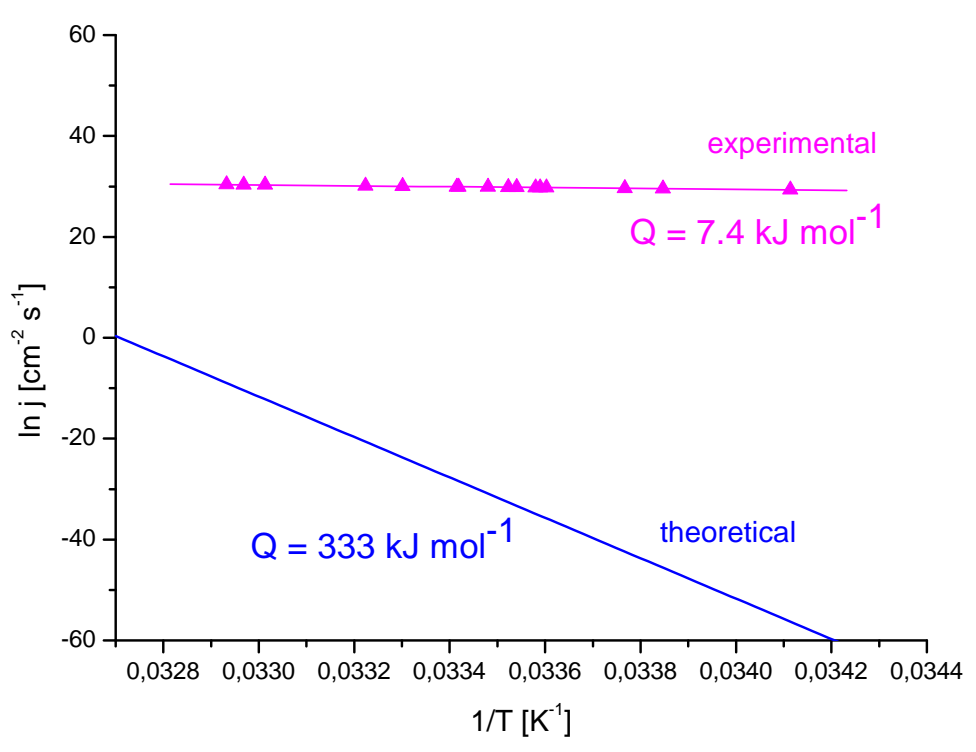

Figure 5.4: Arrhenius plots of the theoretically derived values compared with the experimental values. The slope of the linear fits defines the activation energy $Q$ scaled by the gas constant $R$. The activation energy for the experimental data is distinctively lower than that reported for the diffusion of Co in bulk $\mathrm{Al}_{2} \mathrm{O}_{3}$.

for volume diffusion. The theoretical flux density was calculated using an activation energy of $Q=333 \mathrm{~kJ} \mathrm{~mol}^{-1}$ : if grain boundary diffusion is assumed, one can assume $Q=165 \mathrm{~kJ} \mathrm{~mol}^{-1}$. For, e.g., $T=400^{\circ} \mathrm{C} D_{0}=6.2 \cdot 10^{-5} \mathrm{~cm}^{2} \mathrm{~s}^{-1}$, the diffusion constant is $D=9.69 \cdot 10^{-18} \mathrm{~cm}^{2} \mathrm{~s}^{-1}$ and the diffusion flux density is then $\vec{j}=3.93$. $10^{+12} \mathrm{~cm}^{-2} \mathrm{~s}^{-1}$ (Eq. 5.3). This result is in good agreement with the experimental values for $\vec{j}$ given in Tab. 5.1 .

In order to get more detailed information concerning the activation energy, the experimental and theoretical values of $\vec{j}$ are plotted in a logarithmic scale over the reciprocal temperature (see Fig. 5.4): the slope of the linear fit to the experimental values yields the activation energy $Q$ scaled with the general gas constant $R$. One recognizes that the slope of the theoretical values is distinctively higher than that of the experimental values, as expected from the arguments presented before. An interesting observation however is that the slope of the experimental values delivers an activation energy of only $Q \approx 7.4 \mathrm{~kJ} \mathrm{~mol}^{-1}$. This value is dramatically lower than the value expected for grain boundary diffusion or any other short circuit diffusion. This fact suggests, that the observed segregation effects are not kinetically controlled by thermally activated diffusion processes. More reasonably one has to assume that the segregation occurs due to the presence of a limited amount of material within the barrier right after deposition. That means, that the segregation is limited by the finite material supply, but by the height of the activation energy. This also shows, that the barrier is stable up to the the temperature range of $450^{\circ} \mathrm{C}$ and does not allow any material to intrude into the barrier. In section 4.1.1 it has been shown 


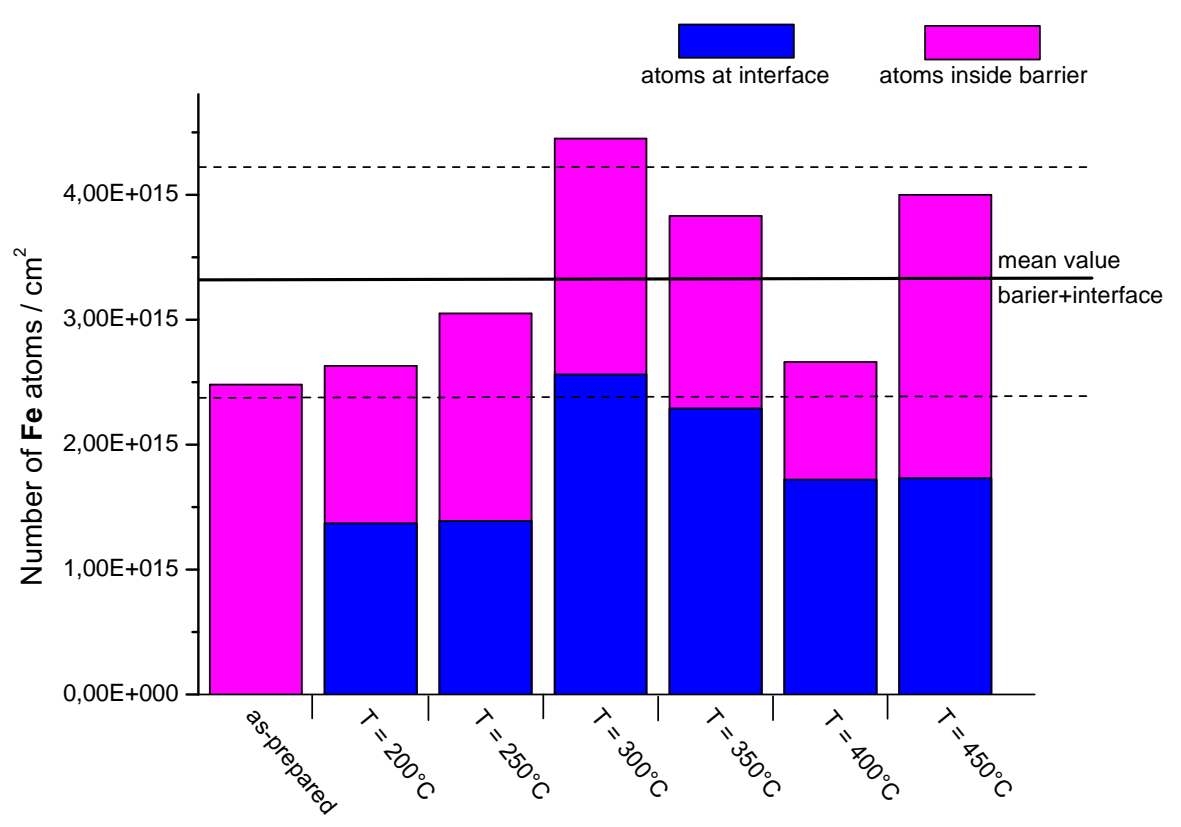

Figure 5.5: Column diagram of the total number of Fe atoms inside the barrier in as-prepared and annealed state. Each column represents a mean value of several measurements. The dashed lines mark the error-limits.

that the barrier is doped with $\mathrm{Ni}$ and Fe right after deposition in the as-prepared state. Only the material that is located inside the barrier right after the deposition contributes to the observed diffusion processes. To prove this idea, the number of segregated Fe atoms at the barrier/Co interface in annealed state is compared with the number of Fe atoms inside the barrier in as-prepared state, as shown in Fig. 5.5 . The columns show a constant number of atoms within acceptable errors, thus the total number of impurity atoms is approximately constant. In consequence the segregation process is feeded by the elements that are already inside the barrier just after deposition. Hence, the diffusion process can not increase with higher increasing annealing temperatures. It is limited by the amount of elements available inside the barrier, rather than by the transport mobility. Although Fe is soluble in Co, the Fe rests at the barrier/Co interface. This may be explained by the presence of a tiny layer of Co-oxide at the barrier/Co interface, that prevents the Fe from intruding into the Co layer.

By contrast the diffusion of $\mathrm{Ni}$ and $\mathrm{Co}$ at annealing temperatures of more than $450^{\circ} \mathrm{C}$ are due to and controlled by diffusion at defects in the barrier structure, as now the transported quantities exceed the amount initially present inside the barrier. 


\subsection{Electrical properties and TMR}

Additionally to TAP analysis, measurements of electrical properties and TMR on planar samples with different barrier thicknesses were obtained. Although the measured resistance of the oxide layer varies along the sample's surface, a real TMR effect could be proven.

The $U / I$ characteristic curve of the sample with the lowest thickness $(d=1 \mathrm{~nm})$ showed a linear behavior that stands for a conventional ohmic resistor. This suggests, that the barrier has a certain amount of pinholes or any other defects, that prevent the $\mathrm{Al}_{2} \mathrm{O}_{3}$ layer from representing a tunnel barrier. On the thickest sample $(d=2 \mathrm{~nm})$ a TMR of at least $3.5 \%$ was measured, while the values for the TMR showed a relatively high variation across the samples surface and reached values of up to $4.5 \%$.

From the $U / I$ characteristic curves and the model given by Brinkman et al. (1970) the barrier structure was characterized. The values for barrier height, barrier asymmetry and barrier thickness showed deviations from an ideal barrier model (see Tab. 4.3). Furthermore these values show also variations with the measuring position upon the samples surface, which indicates a barrier structure with a high surface roughness. Although ion beam sputter deposition is supposed to reveal oxide-barriers that come up with a relatively high surface roughness, it is temporary the only adequate method to fabricate TMR stacks on FIM samples in order to perform a TAP analysis. Deposition methods like plasma oxidation or magnetron sputtering, that are normally applied in industrial applications, are not suitable for preparation of FIM samples. However, these measurements have definitely shown that the stack, that has been deposited on FIM tips by ion beam deposition and analyzed with 3D atom probe tomography, is supposed to have TMR properties. Hence the results and the physical understanding gathered by the results of this work can be transfered to industrial fabricated TMR devices in order to derive a better physical understanding on an atomic scale and to increase the performance and efficiency of TMR devices. 


\section{Chapter 6}

\section{Summary and Outlook}

For the first time controlled measurements of oxide barrier structures using 3D atomprobe tomography have been obtained, if the barrier has a thickness of less than $2.5 \mathrm{~nm}$. The investigated spin-valve system consisting of $\mathrm{Py} / \mathrm{Al}_{2} \mathrm{O}_{3} /$ Co shows an intermixing of components from the Py layer within the oxide barrier. This phenomenon suggests a nano-crystalline structure of $\mathrm{Al}_{2} \mathrm{O}_{3}$ which is in contradiction to several previous publications about the properties of thin $\mathrm{Al}_{2} \mathrm{O}_{3}$ films.

In annealed state, several segregation effects have been observed. At lower annealing temperatures up to $250^{\circ} \mathrm{C}$ Fe segregation at the barrier/Co interface has been observed followed by the formation of breakthrough channels consisting of mainly Fe and a reduction of the intermixing of the Py components inside the barrier beginning at annealing temperatures of $300^{\circ} \mathrm{C}$ and higher. From $450^{\circ} \mathrm{C}$ and higher the electrodes get intermixed, while only $\mathrm{Al}$ atoms within the barrier rest in their initial position within the stack. The interface segregation within the setup can not be explained by volume or grain boundary diffusion, but by thermally induced migration of impurities present inside the barrier already at the outset. Only the mixing of $\mathrm{Fe}, \mathrm{Ni}$ and $\mathrm{Co}$ at annealing temperatures of more than $450^{\circ} \mathrm{C}$ is produced by diffusion at presumably along structured defects in the barrier structure.

The results of the TAP measurements have been confirmed by SIMS and TEM measurements. In addition, the electrical properties and TMR measurements of the spin-valve system deposited on planar samples have been obtained. The samples have shown a clear separation of the top and bottom electrodes and a slight amount of TMR. The condition for that is that the barrier has a thickness in the range of $1.5 \mathrm{~nm}$ to $2.5 \mathrm{~nm}$.

Taking the results of this work into account, several further steps are suggested. First of all there is still the fact that the growth of the layers is supposed to be different on planar substrates than on spheric tips. In order to investigate the structure in more detail and to be sure that the conditions are similar or even identical, the Focused Ion Beam (FIB) technique should be applied to produce FIM tips from planar layer systems. The spin-valve setup may be deposited on Si substrates. Out of these samples FIM tips can be prepared by application of an exactly controlled ion beam to the samples surface. Afterwards the prepared tip can be measured 

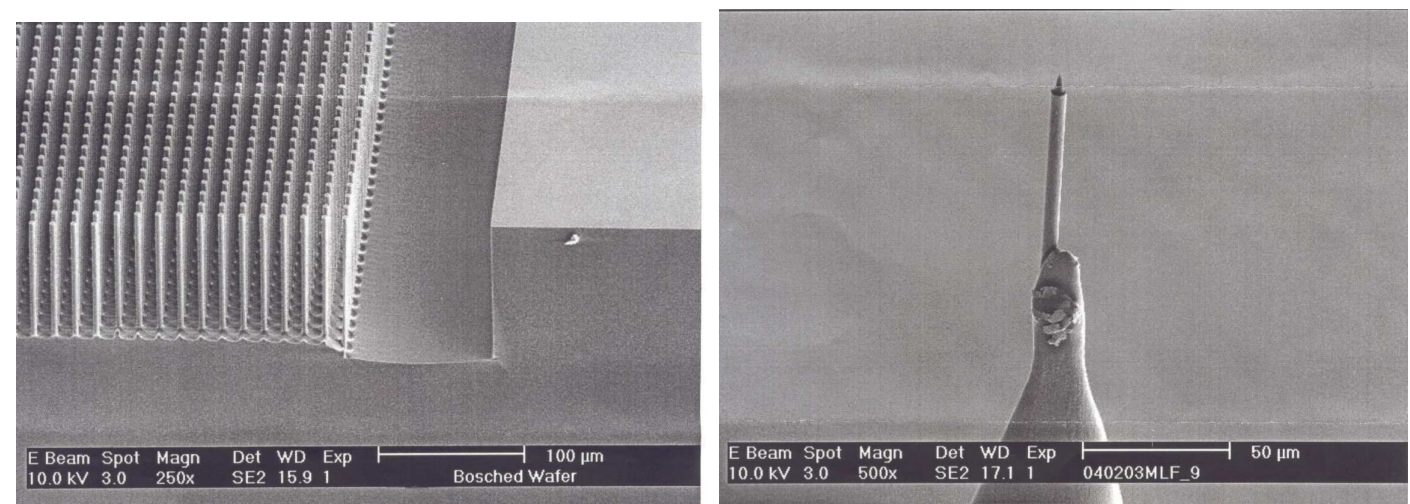

Figure 6.1: Si substrate consisting of tiny columns (left). On this substrate the TMR stack can be deposited and afterwards a spherical FIM tip is prepared out of a column by applying Focused Ion Beam (right).

directly by $3 \mathrm{D}$ atom probe tomography.

Another aspect of interest are the electrical properties of TMR layer systems deposited on tip shaped samples. The FIM tips can be inserted in a Scanning Tunneling Microscope (STM). Normally, the tip in a STM represents the probe and the sample to be investigates is the surface right in front of the tip. In this case the situation would be inverted. STM provides a tip movement with high accuracy in the range of a few $\AA$. Hence the FIM tip can be moved toward the contact electrode, where a voltage can be applied to the tip in order to obtain a $U / I$ characteristic curve or to create electrical breakthrough effects. These structures can afterwards be analyzed in TAP measurements.

Finally, the system can be varied in its composition. In this work it has been shown that investigations of oxide barriers with 3D atom probe tomography are successful. Similarly the investigation of nitride or carbide structures should be possible, which opens a vast field of unsolved problems in material physics. These materials show similar physical properties but a different mechanical behavior. 


\section{List of Tables}

3.1 Overview of the parameters used for the processes in sputter deposition 30

3.2 Overview of the parameters used for SIMS measurement . . . . . . . 41

4.1 Measured oxygen/aluminum ratio inside the barrier. . . . . . . . . . . 51

4.2 Overview of the samples used for electrical characterization . . . . . . 66

4.3 Overview of the results of the fit to the $U / I$ characteristic curves according to the model by Brinkman et al. (1970) in comparison to an ideal barrier. . . . . . . . . . . . . . . . . . . 69

5.1 Overview of the theoretically expected and experimentally derived diffusion data of different elements through the $\mathrm{Al}_{2} \mathrm{O}_{3}$ barrier. . . . . 77 


\section{List of Figures}

2.1 Density of states (DOS) in a non-ferromagnetic and in a ferromagnetic material ...................... . . . . . . . . . . .

2.2 Alignment of the magnetization in two ferromagnetic materials brought into close proximity . . . . . . . . . . . . . . . 11

2.3 Spin polarization inside a non-ferromagnetic interlayer caused by the attached ferromagnet . . . . . . . . . . . . . . . . . 12

2.4 Schematic diagram of the conditions assumed in the model by Slonczewskil. ........................ 15

2.5 Barriers assumed in the Brinkman model . . . . . . . . . . . . . . . . 17

2.6 Fit curves for calculation of the barrier conductance . . . . . . . . . . 19

2.7 Thermal treatment on TMR setups with different barrier thicknesses 21

2.8 Breakthrough voltages for two different sample treatments . . . . . . 22

2.9 Possible distribution of oxygen around the barrier . . . . . . . . . . . 23

3.1 Schematic drawing of the electro-polishing process . . . . . . . . . . 26

3.2 Preparation of TEM samples . . . . . . . . . . . . . . . . . . 26

3.3 The ion beam deposition device . . . . . . . . . . . . . . . . . . 27

3.4 The specimen stage inside the ion beam sputter deposition chamber . 28

3.5 Geometry of the sputter deposition device . . . . . . . . . . . . . . 29

3.6 Scheme of the magnification . . . . . . . . . . . . . . . . . 31

$3.7 \quad$ FIM image of the $\{011\}$-pole of $\mathrm{W}$ at $U=5.6 \mathrm{kV}$ and $T=60 \mathrm{~K}$. . . . 33

3.8 Sketch of the TAP setup in the Institute for Material Physics of the University of Göttingen . . . . . . . . . . . . . . . . . . 34

3.9 The TAP setup in the Institute for Material Physics of the University of Göttingen . . . . . . . . . . . . . . . . . . . 34

3.10 Schematic drawing of functioning principle of the tomographic atom

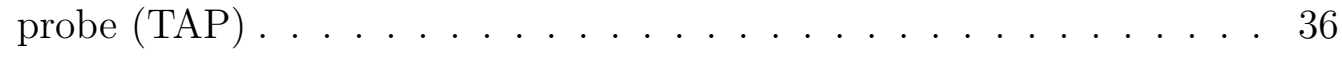


3.11 Geometrical structure of the reconstruction procedure based on initial

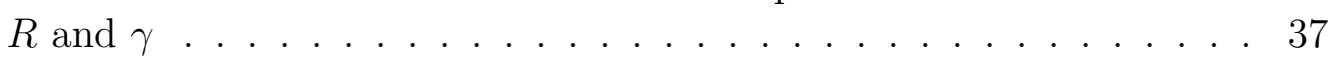

3.12 Field evaporation curve in a multilayer stack with a barrier consisting of Al-oxide . . . . . . . . . . . . . . . . . 38

3.13 The SIMS principle. . . . . . . . . . . . . . . . . . . . . . . . 40

3.14 The SIMS principle in chronological order. . . . . . . . . . . . . . . . 41

3.15 Substrate structure for contact experiments . . . . . . . . . . . . 42

3.16 Principle of the measurement of electric properties and TMR . . . . . 42

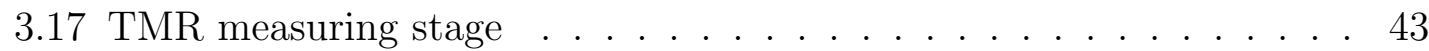

4.1 TAP reconstruction of a layered system with oxide barrier . . . . . . 46

4.2 Mass spectrum of the TAP measurement . . . . . . . . . . . . . . . . 47

4.3 Correction areas and variables . . . . . . . . . . . . . . . . . . . . . . . 48

4.4 Concentration profile including the oxide barrier . . . . . . . . . . . . 49

4.5 Concentration profile including only pure Al . . . . . . . . . . . . . . 50

$4.6 \quad$ 2D-composition maps of the oxygen concentration . . . . . . . . . . 52

4.7 Evolution of the size of the oxide ring . . . . . . . . . . . . . . 53

4.8 Concentration profiles of annealed samples . . . . . . . . . . . . . . . 54

4.9 Two-dimensional Al and Fe composition maps . . . . . . . . . . . . . 55

4.10 The Gibbsian interfacial excess of Fe atoms . . . . . . . . . . . . . . 56

4.11 Iso-concentration surface at $12 \mathrm{at} \%$ (top) of $\mathrm{Fe}$. . . . . . . . . . . 56

4.12 Mass spectrum and the corresponding concentration profile \#1 . . . . 58

4.13 Mass spectrum and the corresponding concentration profile \#2 . . . . 59

4.14 Mass spectrum and the corresponding concentration profile \#3 . . . . 60

4.15 Grain boundary segregation of Co along a Py grain boundary . . . . 61

4.16 TEM images . . . . . . . . . . . . . . . . . . 63

4.17 SIMS profiles of as-prepared samples . . . . . . . . . . . . . . . . 64

4.18 SIMS profiles of an annealed sample. . . . . . . . . . . . . . . 65

4.19 Characteristic magnetic curves . . . . . . . . . . . . . . . . . 67

$4.20 U / I$ characteristic curves . . . . . . . . . . . . . . . . . 68

4.21 TMR characteristic curve . . . . . . . . . . . . . . . . . . . . . 69

5.1 SRIM simulation window . . . . . . . . . . . . . . . . . . . 73 
5.2 Arrhenius plot of Co diffusing into $\mathrm{Al}_{2} \mathrm{O}_{3} \ldots \ldots \ldots \ldots$

5.3 Ellingham diagram of the elements involved in the investigated structure. . . . . . . . . . . . . . . . . . 75

5.4 Arrhenius plots of the theoretically derived values compared with the experimental values $\ldots \ldots \ldots \ldots \ldots \ldots \ldots$

5.5 Column diagram of the total number of Fe atoms inside the barrier in as-prepared and annealed state . . . . . . . . . . . . . . . . 79

$6.1 \quad$ FIB substrate and FIM tip prepared by FIB $\ldots \ldots \ldots \ldots$. . . . . . 82 


\section{Bibliography}

Al-Kassab, T., H. Wollenberger, G. Schmitz, and R. Kirchheim: 2003, Highresolution imaging and spectrometry of materials, Chapt. Tomography by Atom Probe Field Ion Microscopy. Springer Verlag Berlin.

Bankmann, J.: 1998, Untersuchungen zum Einsatz der Sekundärionenmassenspektrometrie bei der Tiefenprofilierung dünner Einzel- und Vielfachschichten. Diploma thesis, Georg-August-Universität Göttingen.

Bas, P., A. Bostel, B. Deconihout, and D. Blavette: 1995, ". Appl. Surface Sci. $87 / 88,298$.

Blavette, D., J. Bostel, J. Sarrau, B. Deconihout, and A. Menand: 1993, '. Nature $\mathbf{3 6 3}, 432$.

Bratovsky, A.: 1997, 'Tunneling of electrons in conventional and half-metallic systems: Towards very large magnetoresistance'. Physical Review B 56, 2344-2347.

Brinkman, W., R. Dynes, and J. Rowell: 1970, 'Tunneling conductance of asymmetrical barriers'. Journal Of Applied Physics 41, 1915-1921.

Fromm, E. and E. Gebhardt: 1976, Gase und Kohlenstoff in Metallen. SringerVerlag.

Grünberg, P., R. Schreiber, Y. Pang, M. Brodsky, and H. Sowers: 1986, 'Layered Magnetic Structures: Evidence for Antiferromagnetic Coupling of Fe Layers across Cr Interlayers'. Physical Review Letters 57, 2442-2445.

Ion-TOF, F.: 1996, TOF SIMS IV. Users Manual.

Jeske, T.: 2001, Nanoanalyse der Frühstadien der Interaktion im System Al/Ni mittels 3D-Atomsondentomographie. $\mathrm{PhD}$ thesis, Georg-August-Universität Göttingen.

Jeurgens, L., W. Sloof, F. Tichelaar, and E. Mittemeijer: 2002, 'Growth kinetics and mechanisms of aluminum-oxide films formed by thermal oxidation of aluminum'. Journal of Applied Physics 92, 1649-1659.

Jullière, M.: 1975, 'Tunneling between ferromagnetic films'. Physical Letters 54A, $225-226$. 
Kluthe, C., T. Al-Kassab, and R. Kirchheim: 2002, 'Tomographic atom probe investigation of $\mathrm{MgO}$ precipitates in silver'. Mat. Sci. Eng. A327, 70-75.

Larson, D., A. Petford-Long, A. Cerezo, A. Morrone, Y. Ma, A. Georgalakis, and P. Clifton: 2003, 'Mechanisms by oxygen acts as a surfant in giant magnetoresistance film growth'. Physical Review $B \mathbf{6 7 .}$

MacLaren, J., X. Zhang, and W. Butler: 1997, 'Validity of the Jullière model of spin-dependent tunneling'. Physical Review B 56(18), 11827-11832.

Meservey, R., P. Tedrow, and P. Fulde: 1970, 'Magnetic Field Splitting of the Quasiparticle States in Superconducting Aluminum Films'. Physical Review Letters 25, 1270-1272.

Miller, M.: 1989, Atomprobe Microanalysis. MRS Pittsburgh.

Moodera, J., L. Kinder, T. Wong, and R. Meservey: 1995, 'Large Magnetoresistance at room temperature in ferromagnetic thin film tunnel junctions'. Physical Review Letters 74, 3273-3276.

Moodera, J., J. Nowak, and R. van de Veerdonk: 1998, 'Interface Magnetism and Spin Wave Scattering in Ferromagnet-Insulator-Ferromagnet Tunnel Junctions'. Physical Review Letters 80, 2941-2944.

Moon, K., Y. Chen, and Y. Huai: 2002, 'PtMn-based spin-dependent tunneling materials with thin alumina barrier fabricated by two-step natural oxidation'. Journal of Applied Physics 91, 7965-7967.

Park, B., J. Bae, and T. Lee: 2002, 'Growth characteristics of Al oxide formed by ozone in magnetic tunnel junctions'. Journal of Applied Physics 91, 8789-8791.

Parkin, S., K. Moon, K. Pettit, D. Smith, R. Dunin-Borkowski, and M. McCartney: 1999a, 'Magnetic tunnel junctions thermally stable to above $300^{\circ} \mathrm{C}$ '. Applied Physics Letters 75, 543-545.

Parkin, S., K. Roche, M. Samant, P. Rice, R. Beyers, R. Scheuerlein, R. O'Sullivan, S. Brown, D. Bucchigano, D. Abraham, Y. Lu, M. Rooks, P. Trouilloud, R. Wanner, and W. Gallagher: 1999b, 'Exchange-biased magnetic tunnel junctions and application to nonvolatile magnetic random access memory (invited)'. Journal of Applied Physics 85, 5828-5833.

Roos, B., P. Beck, S. Demokritov, and B. Hillebrands: 2001, 'Ion oxidation mechanisms controlling the formation of barriers in magnetic tunnel junctions'. Journal of Applied Physics 89, 6656-6658.

Rüsing, J., J. Sebastian, O. Hellman, and D. Seidman: 2000, '. Microsc. Microanal. 6, 445 .

Schleiwies, J. and G. Schmitz: 2002, 'Thin film interaction of $\mathrm{Al} / \mathrm{Ag}$ analyzed by tomographic atom probe'. Mat. Sci. Eng. A327, 94-100. 
Schleiwies, J., G. Schmitz, T. Heitmann, and A. Hütten: 2001, 'Nanoanalysis of $\mathrm{Co} / \mathrm{Cu} / \mathrm{NiFe}$ thin films by tomographic atom probe'. Applied Physics Letters 78, 94-100.

Schmalhorst, J.: 2001, Magnetische, thermische und dielektrische Stabilität von magnetischen Tunnelelementen. Dissertation, Universität Bielefeld.

Schmalhorst, J., H. Brückl, G. Reiss, M. Vieth, G. Gieres, and J. Wecker: 2000, 'Temperature stability of $\mathrm{Co} / \mathrm{Al}_{2} \mathrm{O}_{3}$ '. Journal of Applied Physics 87, 5191-5193.

Schmitz, G.: 2001, Microstructural mechanisms of solid state interactions. habil. thesis, Georg-August-Universität Göttingen.

Simmons, J.: 1963, 'Generalized formula for the electric tunnel effect between similar electrodes seperated by a thin insulating layer'. Journal Of Applied Physics 34, 1793-1803.

Slonczewski, J.: 1989, 'Conductance and exchange coupling of two ferromagnets separated by a tunneling barrier'. Physical Review B 39, 6995-7002.

Sousa, R., J. Sun, V. Soares, P. Freitas, A. Kling, M. da Silva, and J. Soares: 1999, 'Temperature dependance and annealing effects on spin dependent tunnel junctions'. Journal of Applied Physics 85, 5258-5260.

Tsymbal, E. and D. Pettifor: 1998, 'Spin-polarized electron tunneling across a disordered insulator'. Physical Review B 58(1), 432-437.

Ziegler, J., J. Biersack, and U. Littmark: 1985, The Stopping and Range of Ions in Solids. Pergamon Press, New York. 


\section{Acknowledgments}

In this section persons will be mentioned, who made this work and my scientific progress possible.

First of all I would like to thank Prof. Reiner Kirchheim for giving me the opportunity to make my $\mathrm{PhD}$ work in his group at the Institute for Material Physics of the University of Göttingen. Additionally I would like to express my thanks to Prof. Guido Schmitz. During all the time he was a perfect supervisor. If any problems occurred, he always had the time for constructive discussions and enriched my scientific progress with great ideas, although he moved to the University of Münster right after the beginning of my work.

I would also like to thank Dr. Peter Wilbrandt and Mathias Hahn for supporting me in SIMS and SEM analyses. Dr. Dietmar Baither and my former colleague Jens Görlich I would like to thank for support and help in TEM investigations done at the Institute for Material Physics of the University of Münster. Thanks also to Dr. Andreas Hütten and Sven Kämmerer from the University of Bielefeld for performing the electrical contact experiments on my samples.

Great thanks I would like additionally to express to Dr. Rick Hessman from the University Star Observatory of Göttingen for reading and correcting my PhD work. He as a native English speaker and physicist contributed to a better linguistical content. He also helped me to improve my written and spoken English.

I would also like to thank all colleagues from the Institute for Material Physics of the University of Göttingen, especially all those people joining our daily coffeebreaks, like Dr. Christine Borchers, Dr. Hans-Georg Brion, Christine Kuba, Dr. Peter Wilbrandt, Ingoborg Saalfeld and many others. Constantin Ene and Carsten Nowak I would like to thank for rich-full scientific discussions, which supported my efforts in improving my understanding in material physics. I additionally would like to address acknowledgments to all members of the technical staff like those from the workshops for fine mechanics, like Manfred Rothämel, Frank Köhler and Volker Radisch, and electronics - Dieter Bollensen and his staff - as well as to all laboratory assistants and technicians, here especially named Tobias Schulz and Karin Ahlborn. Finally I would like to thank my family, especially my parents Mirjana and Josko Kuduz and my fiancee Maike Sophie Gloddek for supporting me in my work. Also my good friend Markus Waldmann I would like to thank for his support and all the good times and successful radio broadcasts we've had during the recent years. Thanks to everyone! 


\section{Index}

$2 \sigma, 46$

ad-atoms, 38

adsorption, field induced, 37

amorphous structure, 86

annealed state, 62

anodes, 40

Arhenius plot, 92

Arrhenius equation, 87

Arrhenius plot, 87

as-prepared state, 53

atomic volume, 90

back-projection, 43

barrier roughness, 78

Bloch walls, 11

breakthrough channels, 63

breakthrough voltages, 25

Brinkman, model, 21

Buncher (SIMS), 48

carbides, 99

chanel plates, 36

characteristic curve, $U / I, 23,50$

characteristic magnetic curves, 78

cleaning, tip, 34

concentration profile, 57

conductance, 19

cross-section sample preparation, 30

density of states, 9

detector efficiency, 42

detector, two dimensional, 40

diffusion, 87

diffusion flux density, 90

dimple grinding, 30

distribution, binomial, 46

domains, 11

eigenfunction, 18 electron clouds, 38

Ellingham diagram, 87

error, statistic, 46

evaluation volume, 45, 46

excess, Gibbsian, 46, 63

Fermi energy, 11, 15, 23

Fermi level, 15, 38

Fick's law, 90

field emission microscope, 40

field evaporation, 38

field evaporation rate, 44

field ion microscope, 36

Focused Ion Beam (FIB), 98

fringing fields, 11

Giant Magneto-resistance (GMR), 14

Gibbsian excess, 46

grain boundary segregation, 67, 72

imaging gas, 37

in-situ deposition, 35

integrity, oxide layer, 61

interface segregation, 63

Interlayer Exchange Coupling, 12

intermixing, 57, 67, 85

ion beam sputter deposition, 28, 32

ion-polishing, 31

Jullière, model, 15

Kaufmann source, 34

length scale, 73

magneto-resistance, positive, 15

magnetron sputtering, 28

majority-electrons, 11

mass spectrum, 53

material, ferromagnetic, 11

matrix effect, 73 
matrix-effect, 50

MCP, 36

minority electrons, 11

nano-crystalline structure, 86

nitrides, 99

oxidation methods, 60

oxygen distribution, 27

oxygen impurities, 28

parabolic behavior, 23

peak correction, 56

plasma oxidation, 28

polarization, 13

pulse, high voltage, 40

Quantum Confinement Model, 13

ring radius, 62

RKKY model, 11

Ruderman-Kittel-Kasuya-Yosida, 11

Scanning Tunneling Microscope (SEM), 99

Secondary Ion Mass Spectroscopy (SIMS), 31, 73

Secondary Ion Mass Spectroscopy(SIMS), 47

segregation of material, 46

SIMS profiles, 73

Slonczewski, model, 16

solubility of metals, 90

spin operator, 18

spin-up /-down electrons, 11

spin-valve structure, 24

spin-valve system, 97

sputter deposition, 32

SRIM, 85

standard deviation, 46

subsequent oxidation, 35, 84

substrate, 29

TEM analysis, 30

thermal stability, 25

time of flight, 40,48

time-of-flight, 46

TOF detector, 48
Transmission Electron Microscope (TEM), 47

TRIM, 85

Tunnel Magneto-Resistance (TMR), 14

Tunneling Electron Microscope (TEM), 73

two current model, Mott, 14

voltage stability, 25

voltage, best image, 38

Wenzel-Kramer-Brillouin, model, 21 


\section{Lebenslauf}

- Persönliche Daten:

Name: Mario Kuduz.

Geboren am 11. August 1975 in Göttingen.

Nicht verheiratet, keine Kinder.

Beruf: Diplom-Physiker.

Staatsangehörigkeit: Deutsch.

- Ausbildungsweg:

- 1981 - 1985 Grundschule Bovenden.

- 1985 - 1987 Orientierungsstufe Bovenden.

- 1987 - 1994 Otto-Hahn-Gymnasium Göttingen.

- Juli 1994: Abschluß der Schullaufbahn mit bestandenem Abitur.

- März 1995 bis Mai 1996 Zivildienst bei der kath. Kirchengemeinde St. Michael in Göttingen.

- Oktober 1996 - September 1997 Studium im Fach Geologie an der Universität Göttingen.

- Oktober 1997 bis November 2001 Studium im Fach Physik an der Universität Göttingen.

Von September 2000 bis Oktober 2001 Diplomarbeit an der UniversitätsSternwarte Göttingen mit dem Thema "Optische Spektroskopie der galaktischen superweichen Röntgenquelle QR And (= RX J0019.8+2156)". Im Rahmen der Arbeit Aufnahme von Beobachtungsdaten am 3.5m und $1.2 \mathrm{~m}$ Teleskop am Calar Alto (Spanien). Abschluß mit bestandenem Diplom im November 2001.

- Vom 01.01.2002 bis 31.03.2002 wissenschaftlicher Mitarbeiter im Zentrum für Luft- und Raumfahrt (DLR) in Göttingen.

- Seit März 2002 im Rahmen der Promotion mit dem Thema "Nanocharacterisation of magnetoresistant oxide tunnel barrier structures" wissenschaftlicher Mitarbeiter am Institut für Materialphysik der Universität Göttingen. 